\title{
DAHA and iterated torus knots
}

\author{
IVAN CHEREDNIK \\ IVAN DANILENKO
}

\begin{abstract}
The theory of DAHA-Jones polynomials is extended from torus knots to their arbitrary iterations (for any reduced root systems and weights), which includes the polynomiality, duality and other properties of the DAHA superpolynomials. Presumably they coincide with the reduced stable Khovanov-Rozansky polynomials in the case of nonnegative coefficients. The new theory matches well the classical theory of algebraic knots and (unibranch) plane curve singularities; the Puiseux expansion naturally emerges. The corresponding DAHA superpolynomials are expected to coincide with the reduced ones in the Oblomkov-Shende-Rasmussen conjecture upon its generalization to arbitrary dominant weights. For instance, the DAHA uncolored superpolynomials at $a=0, q=1$ are conjectured to provide the Betti numbers of the Jacobian factors (compactified Jacobians) of the corresponding singularities.
\end{abstract}

14H50, 17B45, 20C08, 57M25, 17B22; 20F36, 33D52, 30F10, 55N10

\section{(Introduction}

The theory of DAHA-Jones polynomials of torus knots $[12 ; 22 ; 13]$ is fully extended in this paper to arbitrary iterated knots for any reduced root systems and dominant weights, which includes the polynomiality, duality and other properties of the DAHA superpolynomials. We conjecture the coincidence of the latter with the stable reduced Khovanov-Rozansky polynomials [27; 28; 26; 37] for pseudoalgebraic knots, including all algebraic knots.

A similar connection is also expected with the physics superpolynomials associated with the BPS states (see eg $[15 ; 1 ; 16]$ ) and those related to the Hilbert schemes of plane curve singularities and rational DAHA; see $[33 ; 23 ; 22]$ and references therein. We note that using rational DAHA here is restricted only to the torus knots (and adding colors is generally an open problem).

The new theory matches well the classical theory of algebraic knots and unibranch plane curve singularities, though we see no a priori reasons for this. The Newton pairs in the theory of Puiseux expansion naturally emerge in our approach; see eg [17]. For instance, the iterations that are trivial topologically result in interesting algebraic 
symmetries of the DAHA superpolynomials, for instance, in uniform formulas for the torus knots in the form $T(\mathrm{r}, m \mathrm{r}+\mathrm{s})$ for any $m>0$.

We expect that our superpolynomials add $t$ to the Oblomkov-Shende conjecture [34; 30] and add weights to [33, Conjecture 2] for algebraic knots; see [30, Section 1.5]. The reduced ORS conjecture connects the Poincaré polynomial $\mathscr{P}_{K h R}$ of the triply graded reduced Khovanov-Rozansky homology of an algebraic link with $\mathscr{P}_{\text {alg }}$ describing the cohomology of the nested Hilbert scheme of the corresponding plane curve singularity under the weight filtration. We conjecture them to coincide with the uncolored DAHA superpolynomial $\mathscr{P}_{D A H A}$.

The coincidence with $\mathscr{P}_{K h R}$ is expected for any pseudoalgebraic knots (see above and Conjecture 2.4). The reduction of $\mathscr{P}_{D A H A}$ to the HOMFLYPT polynomials is conjectured for any iterated knots and weights (we prove it for $s l_{2}$ and checked in all the examples). The link $\mathscr{P}_{D A H A} \sim \mathscr{P}_{K h R}$ is a challenge (our examples are mainly for the Khovanov polynomials).

Finding $\mathscr{P}_{K h R}$ is an involved task; one of the reasons is that the skein relations generally cannot be extended from the HOMFLYPT polynomials to the HOMFLYPT homology. The polynomial $\mathscr{P}_{\text {alg }}$ is very sophisticated too. For instance, its portion of minimal $a$-degree requires knowing the so called perverse filtration in the cohomology of the Jacobian factors. Even the Betti numbers of the latter are known only in few cases. See [36, Theorem 22, Conjecture 23] concerning the torus knots and those for Puiseux exponents $(4,2 u, v)$.

By contrast, $\mathscr{P}_{D A H A}$ can be calculated in an entirely formal way without any (theoretical) limitations; we expect that this construction will eventually include arbitrary iterated links. Thus, the main obstacle with stating parts (ii) and (iii) of Conjecture 2.4 below for arbitrary weights is the absence of $\mathscr{P}_{K h R}$ and $\mathscr{P}_{\text {alg }}$ in such a generality. Concerning $\mathscr{P}_{D A H A} \sim \mathscr{P}_{\text {alg }}$, the connection of DAHA with the $K$-theory of the Hilbert schemes of $\mathbb{C}^{2}[41]$ and the affine Springer fibers [45; 49] can be mentioned; the Jacobian factors of isolated plane curve singularities are certain affine Springer fibers [29].

In the examples we calculated, the $t$-coefficients of the DAHA superpolynomials evaluated at $a=0, q=1$ coincide with the Betti numbers (when known) of the corresponding Jacobian factors (compactified Jacobians), which are the ranks of the corresponding cohomology groups. Their sum, the Euler number, can be calculated via the HOMFLYPT polynomial; see [34, Section 7] and [30].

We provide conjectural Betti numbers in the case of Puiseux exponents $(6,8,9)$ and $(6,9,10)$ and quite a few examples beyond the technique used in [36]. In the case of $(6,8,9)$, we calculate formal DAHA counterparts of such Betti numbers for $2 \omega_{1}$ and 
$\omega_{2}$. Also, we do this for several uncolored nonalgebraic iterated knots; for instance, we extend the family $(4,6, v \geq 7)$ from [36] to pseudoalgebraic $v=3,5$.

In all considered examples (not only for the pseudoalgebraic iterated knots), our superpolynomials reduce to the corresponding HOMFLYPT polynomials under $t \mapsto q$, $a \mapsto-a$ for arbitrary dominant weights and any iterated knots. They match well the (uncolored) Khovanov polynomials (for $s l_{2}$ ) in the sense of [12, Conjecture 3.7] for all pseudoalgebraic knots we considered; recall that the latter are defined as those with nonnegative coefficients of (uncolored) $\mathscr{P}_{D A H A}$. It is possible that such a connection can be extended to any iterated knots; see Section 4.1.

We note that our paper was triggered by paper [40], though it is restricted to $A_{1}$ and we do not quite understand its approach. The Newton pairs and other features of our construction do not appear in [40]. Also, the polynomials $J_{n}$ there have significant $q, t$-denominators (even in the uncolored case); the polynomiality of DAHA-Jones polynomials is the key in our theory. The $J_{2}$-polynomials for $\operatorname{Cab}( \pm 5,2) T(3,2)$ from [40, Section 5.2] are very different from our ones in these cases (and we do not understand how they were obtained); see (4-3) below. The first part of [40] is devoted to the skein modules, which continues [5]; this direction is of obvious importance.

\section{DAHA and Macdonald polynomials}

\subsection{Affine root systems}

Let $R=\{\alpha\} \subset \mathbb{R}^{n}$ be a root system of type $A_{n}, \ldots, G_{2}$ with respect to a euclidean form $\left(z, z^{\prime}\right)$ on $\mathbb{R}^{n} \ni z, z^{\prime}, W$ the Weyl group generated by the reflections $s_{\alpha}, R_{+}$the set of positive roots corresponding to fixed simple roots $\alpha_{1}, \ldots, \alpha_{n}$, and $R_{-}=-R_{+}$. The form is normalized by the condition $(\alpha, \alpha)=2$ for short roots. The weight lattice is $P=\bigoplus_{i=1}^{n} \mathbb{Z} \omega_{i}$, where $\left\{\omega_{i}\right\}$ are fundamental weights: $\left(\omega_{i}, \alpha_{j}^{\vee}\right)=\delta_{i j}$ for the coroots $\alpha^{\vee}=2 \alpha /(\alpha, \alpha)$. The root lattice is $Q=\bigoplus_{i=1}^{n} \mathbb{Z} \alpha_{i}$. Replacing $\mathbb{Z}$ by $\mathbb{Z}_{ \pm}=\{m \in \mathbb{Z}, \pm m \geq 0\}$, we obtain $P_{ \pm}, Q_{ \pm}$. See eg [7] or [10].

Setting $v_{\alpha}:=(\alpha, \alpha) / 2$, the vectors $\tilde{\alpha}=\left[\alpha, v_{\alpha} j\right] \in \mathbb{R}^{n} \times \mathbb{R} \subset \mathbb{R}^{n+1}$ for $\alpha \in R, j \in \mathbb{Z}$ form the twisted affine root system $\widetilde{R} \supset R\left(z \in \mathbb{R}^{n}\right.$ are identified with $\left.[z, 0]\right)$. We add $\alpha_{0}:=[-\vartheta, 1]$ to the simple roots for the maximal short root $\vartheta \in R_{+}$. The corresponding set $\widetilde{R}_{+}$of positive roots is $R_{+} \cup\left\{\left[\alpha, v_{\alpha} j\right], \alpha \in R, j>0\right\}$.

The set of the indices of the images of $\alpha_{0}$ by all automorphisms of the affine Dynkin diagram will be denoted by $O\left(O=\{0\}\right.$ for $\left.E_{8}, F_{4}, G_{2}\right)$. Let $O^{\prime}:=\{r \in O, r \neq 0\}$. The elements $\omega_{r}$ for $r \in O^{\prime}$ are minuscule weights, defined by the inequalities $\left(\omega_{r}, \alpha^{\vee}\right) \leq 1$ for all $\alpha \in R_{+}$. We set $\omega_{0}=0$ for the sake of uniformity. 
Affine Weyl groups Given $\widetilde{\alpha}=\left[\alpha, v_{\alpha} j\right] \in \widetilde{R}$ and $b \in P$, let

$$
s_{\widetilde{\alpha}}(\widetilde{z})=\widetilde{z}-\left(z, \alpha^{\vee}\right) \tilde{\alpha}, \quad b^{\prime}(\widetilde{z})=[z, \zeta-(z, b)]
$$

for $\widetilde{z}=[z, \zeta] \in \mathbb{R}^{n+1}$. The affine Weyl group $\widetilde{W}=\left\langle s \widetilde{\alpha}, \widetilde{\alpha} \in \widetilde{R}_{+}\right\rangle$is the semidirect product $W \ltimes Q$ of its subgroups $W=\left\langle s_{\alpha}, \alpha \in R_{+}\right\rangle$and $Q$, where $\alpha$ is identified with

$$
s_{\alpha} S_{\left[\alpha, v_{\alpha}\right]}=s_{\left[-\alpha, v_{\alpha}\right]} S_{\alpha} \quad \text { for } \alpha \in R .
$$

The extended Weyl group $\widehat{W}$ is $W \ltimes P$, where the corresponding action is

$$
(w b)([z, \zeta])=[w(z), \zeta-(z, b)] \quad \text { for } w \in W, b \in P .
$$

It is isomorphic to $\widetilde{W} \ltimes \Pi$ for $\Pi:=P / Q$. The latter group consists of $\pi_{0}=$ id and the images $\pi_{r}$ of minuscule $\omega_{r}$ in $P / Q$.

The group $\Pi$ is naturally identified with the subgroup of $\widehat{W}$ of the elements of the length zero; the length is defined as

$$
l(\widehat{w})=|\lambda(\widehat{w})| \quad \text { for } \lambda(\widehat{w}):=\widetilde{R}_{+} \cap \widehat{w}^{-1}\left(-\widetilde{R}_{+}\right) .
$$

One has $\omega_{r}=\pi_{r} u_{r}$ for $r \in O^{\prime}$, where $u_{r}$ is the element $u \in W$ of minimal length such that $u\left(\omega_{r}\right) \in P_{-}$.

Setting $\widehat{w}=\pi_{r} \widetilde{w} \in \widehat{W}$ for $\pi_{r} \in \Pi$ and $\widetilde{w} \in \widetilde{W}, l(\widehat{w})$ coincides with the length of any reduced decomposition of $\tilde{w}$ in terms of the simple reflections $s_{i}$ for $0 \leq i \leq n$.

\subsection{Definition of DAHA}

We follow $[13 ; 12 ; 10]$. Let $\mathrm{m}$ be the least natural number such that $(P, P)=(1 / \mathrm{m}) \mathbb{Z}$. Thus $\mathrm{m}=|\Pi|$ with the following exceptions: $\mathrm{m}=2$ for $D_{2 k}$, and $\mathrm{m}=1$ for $B_{2 k}$ and $C_{k}$.

The double affine Hecke algebra, DAHA, depends on the parameters $q$ and $t_{v}\left(v \in\left\{v_{\alpha}\right\}\right)$ and will be defined over the ring $\mathbb{Z}_{q, t}:=\mathbb{Z}\left[q^{ \pm 1 / \mathrm{m}}, t_{v}^{ \pm 1 / 2}\right]$ formed by polynomials in terms of $q^{ \pm 1 / \mathrm{m}}$ and $\left\{t_{v}^{1 / 2}\right\}$. Note that the coefficients of the Macdonald polynomials will belong to $\mathbb{Q}\left(q, t_{v}\right)$.

For $\tilde{\alpha}=\left[\alpha, v_{\alpha} j\right] \in \widetilde{R}, 0 \leq i \leq n$, we set

$$
t_{\widetilde{\alpha}}=t_{\alpha}=t_{v_{\alpha}}=q_{\alpha}^{k_{\nu}}, \quad q_{\widetilde{\alpha}}=q^{v_{\alpha}}, \quad t_{i}=t_{\alpha_{i}}, \quad q_{i}=q_{\alpha_{i}} .
$$

Also, using here (and below) sht and lng instead of $v$, we set

$$
\rho_{k}:=\frac{1}{2} \sum_{\alpha>0} k_{\alpha} \alpha=k_{\mathrm{sht}} \rho_{\mathrm{sht}}+k_{\mathrm{lng}} \rho_{\mathrm{lng}}, \quad \rho_{\nu}=\frac{1}{2} \sum_{\nu_{\alpha}=v} \alpha=\sum_{\nu_{i}=v, i>0} \omega_{i} .
$$


For pairwise commutative $X_{1}, \ldots, X_{n}$,

$$
\begin{aligned}
X_{\tilde{b}}:=\prod_{i=1}^{n} X_{i}^{l_{i}} q^{j} \quad \text { if } \tilde{b}=[b, j], \quad \widehat{w}\left(X_{\tilde{b}}\right)=X_{\widehat{w}(\tilde{b})}, & \\
\text { where } b & =\sum_{i=1}^{n} l_{i} \omega_{i} \in P, \quad j \in \frac{1}{m} \mathbb{Z}, \quad \widehat{w} \in \widehat{W} .
\end{aligned}
$$

For instance, $X_{0}:=X_{\alpha_{0}}=q X_{\vartheta}^{-1}$.

Recall that $\omega_{r}=\pi_{r} u_{r}$ for $r \in O^{\prime}$ (see above). We will use that $\pi_{r}^{-1}$ is $\pi_{\iota(i)}$, where $\iota$ is the standard involution of the nonaffine Dynkin diagram, induced by $\alpha_{i} \mapsto-w_{0}\left(\alpha_{i}\right)$. Generally, $\iota(b)=-w_{0}(b)=b^{\iota}$, where $w_{0}$ is the longest element in $W$. Finally, we set $m_{i j}=2,3,4,6$ when the number of links between $\alpha_{i}$ and $\alpha_{j}$ in the affine Dynkin diagram is $0,1,2,3$, respectively.

Definition 1.1 The double affine Hecke algebra $\mathcal{H}$ is generated over $\mathbb{Z}_{q, t}$ by the elements $\left\{T_{i} \mid 0 \leq i \leq n\right\}$, pairwise commutative $\left\{X_{b} \mid b \in P\right\}$ satisfying (1-3), and the group $\Pi$, where the following relations are imposed:
(o) $\left(T_{i}-t_{i}^{1 / 2}\right)\left(T_{i}+t_{i}^{-1 / 2}\right)=0$
for $0 \leq i \leq n$.
(i) $T_{i} T_{j} T_{i} \cdots=T_{j} T_{i} T_{j} \cdots$
with $m_{i j}$ factors on each side.
(ii) $\pi_{r} T_{i} \pi_{r}^{-1}=T_{j}$
(iii) $T_{i} X_{b}=X_{b} X_{\alpha_{i}}^{-1} T_{i}^{-1}$ if $\pi_{r}\left(\alpha_{i}\right)=\alpha_{j}$.
(iv) $T_{i} X_{b}=X_{b} T_{i} \quad$ if $\left(b, \alpha_{i}^{\vee}\right)=0$ if $\left(b, \alpha_{i}^{\vee}\right)=1$, for $0 \leq i \leq n$.
(v) $\pi_{r} X_{b} \pi_{r}^{-1}=X_{\pi_{r}(b)}=X_{u_{r}^{-1}(b)} q^{\left(\omega_{\iota(r)}, b\right)}$ for $0 \leq i \leq n$. for $r \in O^{\prime}$.

Given $\widetilde{w} \in \widetilde{W}$ and $r \in O$, the product

$$
T_{\pi_{r} \tilde{w}}:=\pi_{r} T_{i_{l}} \cdots T_{i_{1}}, \quad \text { where } \tilde{w}=s_{i_{l}} \cdots s_{i_{1}} \text { for } l=l(\widetilde{w}),
$$

does not depend on the choice of the reduced decomposition. Moreover,

$$
T_{\widehat{v}} T_{\widehat{w}}=T_{\widehat{v} \hat{w}} \quad \text { whenever } l(\hat{v} \widehat{w})=l(\widehat{v})+l(\widehat{w}) \text { for } \widehat{v}, \widehat{w} \in \widehat{W} .
$$

In particular, we arrive at the pairwise commutative elements

$$
Y_{b}:=\prod_{i=1}^{n} Y_{i}^{l_{i}}, \quad \text { if } b=\sum_{i=1}^{n} l_{i} \omega_{i} \in P, \quad \text { where } Y_{i}:=T_{\omega_{i}}, b \in P .
$$

When acting in the polynomial representation $\mathcal{V}$ (see below), they are called difference Dunkl operators. Their orbit sums (symmetrizations) $\sum_{w \in W} Y_{w(b)}$ then become the Macdonald operators and their generalizations upon acting on symmetric Laurent polynomials. 


\subsection{The automorphisms}

The following maps can be (uniquely) extended to automorphisms of $\mathcal{H H}$, where $q^{1 /(2 \mathrm{~m})}$ must be added to $\mathbb{Z}_{q, t}$ (see [10, (3.2.10-15)]):

$$
\begin{aligned}
& \tau_{+}: X_{b} \mapsto X_{b}, \quad T_{i} \mapsto T_{i}(i>0), \quad Y_{r} \mapsto X_{r} Y_{r} q^{-\left(\omega_{r}, \omega_{r}\right) / 2}, \\
& \tau_{+}: T_{0} \mapsto q^{-1} X_{\vartheta} T_{0}^{-1}, \quad \pi_{r} \mapsto q^{-\left(\omega_{r}, \omega_{r}\right) / 2} X_{r} \pi_{r}\left(r \in O^{\prime}\right), \\
& \tau_{-}: Y_{b} \mapsto Y_{b}, \quad T_{i} \mapsto T_{i}(i \geq 0), \quad X_{r} \mapsto Y_{r} X_{r} q^{\left(\omega_{r}, \omega_{r}\right) / 2}, \\
& \tau_{-}\left(X_{\vartheta}\right)=q T_{0} X_{\vartheta}^{-1} T_{s_{\vartheta}}^{-1} ; \quad \sigma:=\tau_{+} \tau_{-}^{-1} \tau_{+}=\tau_{-}^{-1} \tau_{+} \tau_{-}^{-1}, \\
& \sigma\left(X_{b}\right)=Y_{b}^{-1}, \quad \sigma\left(Y_{b}\right)=T_{w_{0}}^{-1} X_{b^{c}}^{-1} T_{w_{0}}, \quad \sigma\left(T_{i}\right)=T_{i}(i>0) .
\end{aligned}
$$

These automorphisms fix $t_{v}, q$ and their fractional powers, as well as the anti-involution

$$
\varphi: X_{b} \mapsto Y_{b}^{-1}, \quad Y_{b} \mapsto X_{b}^{-1}, \quad T_{i} \mapsto T_{i} \quad(1 \leq i \leq n) .
$$

We will also need the involution

$$
\eta: T_{i} \mapsto T_{i}^{-1}, \quad X_{b} \mapsto X_{b}^{-1}, \quad \pi_{r} \mapsto \pi_{r} \quad(0 \leq i \leq n),
$$

which "conjugates" $t$ and $q$, namely $t_{v}^{1 /(2 \mathrm{~m})} \mapsto t_{\nu}^{-1 /(2 \mathrm{~m})}$ and $q^{1 /(2 \mathrm{~m})} \mapsto q^{-1 /(2 \mathrm{~m})}$. As above, $b \in P$ and $r \in O^{\prime}$. The involution $\eta$ extends the Kazhdan-Lusztig involution in the affine Hecke theory; see [10, (3.2.19-22)]. Note that

$$
\varphi \tau_{ \pm} \varphi=\tau_{\mp}=\sigma \tau_{ \pm}^{-1} \sigma^{-1}, \quad \eta \tau_{ \pm} \eta=\tau_{ \pm}^{-1} .
$$

Let us list the matrices corresponding to the automorphisms and anti-automorphisms above upon the natural projection onto $\mathrm{GL}_{2}(\mathbb{Z})$, corresponding to $t_{v}^{1 /(2 \mathrm{~m})}=1=q^{1 /(2 \mathrm{~m})}$. The matrix $\left(\begin{array}{ll}\alpha & \beta \\ \gamma & \delta\end{array}\right)$ will represent the map

$$
X_{b} \mapsto X_{b}^{\alpha} Y_{b}^{\gamma}, \quad Y_{b} \mapsto X_{b}^{\beta} Y_{b}^{\delta}
$$

for $b \in P$. One has

$\tau_{+} \mapsto\left(\begin{array}{ll}1 & 1 \\ 0 & 1\end{array}\right), \quad \tau_{-} \mapsto\left(\begin{array}{ll}1 & 0 \\ 1 & 1\end{array}\right), \quad \sigma \mapsto\left(\begin{array}{rr}0 & 1 \\ -1 & 0\end{array}\right), \quad \varphi \mapsto\left(\begin{array}{rr}0 & -1 \\ -1 & 0\end{array}\right), \quad \eta \mapsto\left(\begin{array}{rr}-1 & 0 \\ 0 & 1\end{array}\right)$.

Projective $\mathbf{G L}_{2}$ We define the projective $\mathrm{GL}_{2}^{\wedge}(\mathbb{Z})$ as the group generated by $\tau_{ \pm}$and $\eta$ subject to the relations $\tau_{+} \tau_{-}^{-1} \tau_{+}=\tau_{-}^{-1} \tau_{+} \tau_{-}^{-1}, \eta^{2}=1$ and $\eta \tau_{ \pm} \eta=\tau_{ \pm}^{-1}$. The span of $\tau_{ \pm}$is the projective $\operatorname{PSL}_{2}^{\wedge}(\mathbb{Z})$ (due to Steinberg), which is isomorphic to the braid group $B_{3}$. 


\subsection{Macdonald polynomials}

Following [10], we use the PBW theorem to express any $H \in \mathcal{H}$ in the form $\sum_{a, w, b} c_{a, w, b} X_{a} T_{w} Y_{b}$ for $w \in W$ and $a, b \in P$ (this presentation is unique). Then we substitute as follows:

$$
\{\}_{e v}: X_{a} \mapsto q^{-\left(\rho_{k}, a\right)}, \quad Y_{b} \mapsto q^{\left(\rho_{k}, b\right)}, \quad T_{i} \mapsto t_{i}^{1 / 2} .
$$

Polynomial representation The functional $\mathcal{H} \ni H \mapsto\{H\}_{\text {ev }}$, called coinvariant, acts via the projection $H \mapsto H \Downarrow:=H(1)$ of $\mathcal{H}$ onto the polynomial representation $\mathcal{V}$, which is the $\mathcal{H}$-module induced from the one-dimensional character $T_{i}(1)=$ $t_{i}^{-1 / 2}=Y_{i}(1)$ for $1 \leq i \leq n$ and $T_{0}(1)=t_{0}^{-1 / 2}$. Recall that $t_{0}=t_{\text {sht }} ;$ see $[10 ; 12 ; 13]$ here and below.

In detail, the polynomial representation $\mathcal{V}$ is isomorphic to $\mathbb{Z}_{q, t}\left[X_{b}\right]$ as a vector space, and the action of $T_{i}(0 \leq i \leq n)$ there is given by the Demazure-Lusztig operators:

$$
T_{i}=t_{i}^{1 / 2} s_{i}+\left(t_{i}^{1 / 2}-t_{i}^{-1 / 2}\right)\left(X_{\alpha_{i}}-1\right)^{-1}\left(s_{i}-1\right) \quad \text { for } 0 \leq i \leq n .
$$

The elements $X_{b}$ become the multiplication operators and $\pi_{r}\left(r \in O^{\prime}\right)$ act via the general formula $\widehat{w}\left(X_{b}\right)=X_{\widehat{w}(b)}$ for $\widehat{w} \in \widehat{W}$. Note that $\tau_{-}$and $\eta$ naturally act in the polynomial representation. See formula $[13,(1.37)]$. For the latter,

(1-14) $\eta(f)=f^{\star}, \quad$ where $X_{b}^{\star}=X_{-b},\left(q^{v}\right)^{\star}=q^{-v},\left(t^{v}\right)^{\star}=t^{-v}$ for $v \in \mathbb{Q}$.

Concerning the action of $\tau_{-}$, see eg formula [13, (1.37)]. Also,

$$
\{\varphi(H)\}_{e v}=\{H\}_{e v}, \quad\{\eta(H)\}_{e v}=\{H\}_{e v}^{\star} .
$$

Macdonald polynomials The Macdonald polynomials $P_{b}(X)$ for $b \in P_{+}$(they are due to Kadell for the classical root systems) are uniquely defined as follows. For $c \in P$, let $c_{+}$be a unique element such that $c_{+} \in W(c) \cap P_{+}$. Given $b \in P_{+}$and assuming that $b_{+} \neq c_{+} \in b-Q_{+}$,

$$
P_{b}-\sum_{a \in W(b)} X_{a} \in \bigoplus_{c} \mathbb{Q}\left(q, t_{v}\right) X_{c} \quad \text { and } \quad C T\left(P_{b} X_{c^{\iota}} \mu(X ; q, t)\right)=0
$$

where

$$
\mu(X ; q, t):=\prod_{\alpha \in R_{+}} \prod_{j=0}^{\infty} \frac{\left(1-X_{\alpha} q_{\alpha}^{j}\right)\left(1-X_{\alpha}^{-1} q_{\alpha}^{j+1}\right)}{\left(1-X_{\alpha} t_{\alpha} q_{\alpha}^{j}\right)\left(1-X_{\alpha}^{-1} t_{\alpha} q_{\alpha}^{j+1}\right)} .
$$

Here, $C T$ is the constant term; $\mu$ is considered a Laurent series of $X_{b}$ with the coefficients expanded in terms of positive powers of $q$. The coefficients of $P_{b}$ belong 
to the field $\mathbb{Q}\left(q, t_{v}\right)$. One has

$$
P_{b}\left(X^{-1}\right)=P_{b^{\iota}}(X)=P_{b}^{\star}(X), \quad P_{b}\left(q^{-\rho_{k}}\right)=P_{b}\left(q^{\rho_{k}}\right)=\left(P_{b}\left(q^{-\rho_{k}}\right)\right)^{\star},
$$

where

$$
\left.\left(P_{b}\left(q^{-\rho_{k}}\right)\right)^{\star}=q^{-\left(\rho_{k}, b\right)} \prod_{\alpha>0}^{\left(\alpha^{\vee}, b\right)-1} \prod_{j=0}^{\left(-1-q_{\alpha}^{j} t_{\alpha} X_{\alpha}\left(q^{\rho_{k}}\right)\right.} \frac{\left(q_{\alpha}^{j} X_{\alpha}\left(q^{\rho_{k}}\right)\right.}{1-}\right) .
$$

Recall that $\iota(b)=b^{\iota}=-w_{0}(b)$ for $b \in P$ and see formula [10, (3.3.23)]. We set $P_{b}^{\circ}:=P_{b} / P_{b}\left(q^{-\rho_{k}}\right)$ for $b \in P_{+}$; this is the so called spherical normalization.

DAHA provides an important alternative (operator) approach to $P$-polynomials; namely, they satisfy the (defining) relations

$$
L_{f}\left(P_{b}\right)=f\left(q^{-\rho_{k}-b}\right) P_{b}, \quad \text { where } b \in P_{+}, L_{f}:=f\left(X_{a} \mapsto Y_{a}\right),
$$

for any symmetric ( $W$-invariant) polynomial $f \in \mathbb{C}\left[X_{a}, a \in P\right]^{W}$.

\section{DAHA-Jones theory}

\subsection{Iterated torus knots}

The torus knots $T(\mathrm{r}, \mathrm{s})$ are defined for any integers assuming that $\operatorname{gcd}(\mathrm{r}, \mathrm{s})=1$. One has the symmetries $T(\mathrm{r}, \mathrm{s})=T(\mathrm{~s}, \mathrm{r})=T(-\mathrm{r},-\mathrm{s})$, where we use "=" for the ambient isotopy equivalence. Also $T(\mathrm{r}, \mathrm{s})=\bigcirc$ if $|\mathrm{r}| \leq 1$ or $|\mathrm{s}| \leq 1$ for the unknot, denoted by $\bigcirc$ here and below. See eg $[38 ; 17]$ or the Knot Atlas for the details and the corresponding theory of the invariants.

The iterated torus knots, denoted in this paper by $\mathcal{T}(\vec{r}, \vec{s})$, will be associated with two sequences of integers of any signs:

$$
\vec{r}=\left\{r_{1}, \ldots r_{\ell}\right\}, \vec{s}=\left\{s_{1}, \ldots s_{\ell}\right\} \quad \text { such that } \operatorname{gcd}\left(r_{i}, s_{i}\right)=1 .
$$

We will denote by $\ell$ the length of $\vec{r}$ and $\vec{s}$. The pairs $\left\{r_{i}, s_{i}\right\}$ will become characteristic or Newton pairs for algebraic knots. We will use this name, but generally consider arbitrary iterated knots in this paper.

These are combinatorial data. The (topological) definition of iterated torus knots requires one more sequence:

$$
\mathrm{a}_{1}=\mathrm{s}_{1}, \quad \mathrm{a}_{i}=\mathrm{a}_{i-1} \mathrm{r}_{i-1} \mathrm{r}_{i}+\mathrm{s}_{i}(i=2, \ldots, m) .
$$

See eg [17] and [36]. Then, in terms of the cabling defined below,

$$
\mathcal{T}(\overrightarrow{\mathrm{r}}, \overrightarrow{\mathrm{s}}):=\operatorname{Cab}(\overrightarrow{\mathrm{a}}, \overrightarrow{\mathrm{r}})(\bigcirc)=\left(\operatorname{Cab}\left(\mathrm{a}_{\ell}, \mathrm{r}_{\ell}\right) \cdots \operatorname{Cab}\left(\mathrm{a}_{2}, \mathrm{r}_{2}\right)\right)\left(T\left(\mathrm{a}_{1}, \mathrm{r}_{1}\right)\right) .
$$


Note that the first iteration (application of $C a b)$ is for $\left\{a_{1}, r_{1}\right\}$ (not for the last pair!). Recall that $\bigcirc$ is the unknot and knots are considered up to ambient isotopy (we use "=" for it).

Cabling The cabling $\operatorname{Cab}(\mathrm{a}, \mathrm{b})(K)$ of any oriented knot $K$ in (oriented) $S^{3}$ is defined as follows; see eg [32; 17] and references therein. We consider a small 2-dimensional torus around $K$ and put there the torus knot $T(\mathrm{a}, \mathrm{b})$ in the direction of $K$, which is $\operatorname{Cab}(\mathrm{a}, \mathrm{b})(K)$ (up to ambient isotopy). We will sometimes set $\operatorname{Cab}(\vec{a}, \vec{r})=\operatorname{Cab}(\vec{a}, \vec{r})(\bigcirc)$.

This procedure depends on the order of a, b and orientation of $K$. We choose the latter in the standard way (compatible with almost all sources, including the Mathematica package "KnotTheory"); the parameter a gives the number of turns around $K$. This construction also depends on the framing of the cable knots; we take the natural one, associated with the parallel copy of the torus where a given cable knot sits (its parallel copy has zero linking number with this knot).

By construction, $\operatorname{Cab}(\mathrm{a}, 0)(K)=\bigcirc$ and $\operatorname{Cab}(\mathrm{a}, 1)(K)=K$ for any knot $K$ and $\mathrm{a} \neq 0$. Accordingly, we have the following reduction cases:

(2-4) When $\mathrm{r}_{i}=0(i<\ell)$,

$$
\mathcal{T}(\overrightarrow{\mathrm{r}}, \overrightarrow{\mathrm{s}})=\mathcal{T}\left(\left\{\mathrm{r}_{i+1}, \ldots, \mathrm{r}_{\ell}\right\},\left\{\mathrm{s}_{i+1}, \ldots, \mathrm{s}_{\ell}\right\}\right) \text { and } \mathcal{T}(\overrightarrow{\mathrm{r}}, \overrightarrow{\mathrm{s}})=\bigcirc \text { for } i=\ell .
$$

(2-5) When $\mathrm{r}_{i}=1, \mathrm{~s}_{i}=0$,

$$
\mathcal{T}(\vec{r}, \vec{s})=\mathcal{T}\left(\left\{r_{1}, \ldots, r_{i-1}, r_{i+1}, \ldots, r_{\ell}\right\},\left\{s_{1}, \ldots, s_{i-1}, s_{i+1}, \ldots, s_{\ell}\right\}\right) .
$$

(2-6) When $\mathrm{r}_{i}=1, \mathrm{~s}_{i} \neq 0$,

$$
\mathcal{T}(\overrightarrow{\mathrm{r}}, \overrightarrow{\mathrm{s}})=\mathcal{T}\left(\left\{\mathrm{r}_{1}, \ldots, \mathrm{r}_{i-1}, \mathrm{r}_{i+1}, \ldots, \mathrm{r}_{\ell}\right\},\left\{\ldots, \mathrm{s}_{i-1}, \mathrm{~s}_{i+1}^{\prime}, \mathrm{s}_{i+2}, \ldots\right\}\right),
$$

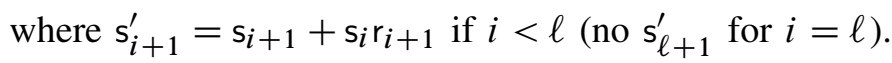

Let us comment on the last relation; see (2-2). Since $r_{i}=1$, one has

$$
\begin{aligned}
\mathrm{a}_{i}=\mathrm{a}_{i-1} \mathrm{r}_{i-1}+\mathrm{s}_{i}, \quad \mathrm{a}_{i+1} & =\mathrm{a}_{i} \mathrm{r}_{i+1}+\mathrm{s}_{i+1} \\
& =\mathrm{a}_{i-1} \mathrm{r}_{i-1} \mathrm{r}_{i+1}+\left(\mathrm{s}_{i+1}+\mathrm{s}_{i} \mathrm{r}_{i+1}\right) .
\end{aligned}
$$

The pairs $\left\{r_{i}, a_{i}\right\}$ are sometimes called topological (cable); the isotopy equivalence of algebraic links generally can be seen only at the level of $r$, a-parameters (not at the level of the Newton or Puiseux pairs).

Also, $T(\mathrm{r}, \mathrm{s})=T(\mathrm{~s}, \mathrm{r})$, which results in the transposition property:

$$
\mathcal{T}(\vec{r}, \vec{s})=\mathcal{T}\left(\left\{s_{1}, r_{2}, \ldots, r_{\ell}\right\},\left\{r_{1}, s_{2}, \ldots, s_{\ell}\right\}\right)
$$


The counterparts of these properties hold for the DAHA-Jones polynomials, though due to entirely algebraic reasons.

Switching from $K$ to its mirror image, denoted by $K^{\star}$, one has

$$
\begin{aligned}
\operatorname{Cab}(\mathrm{a}, \mathrm{b})\left(K^{\star}\right) & =(\operatorname{Cab}(-\mathrm{a}, \mathrm{b})(K))^{\star} \quad \text { for any a, b with } \operatorname{gcd}(\mathrm{a}, \mathrm{b})=1, \\
\operatorname{Cab}(-\overrightarrow{\mathrm{a}}, \overrightarrow{\mathrm{r}}) & =(\operatorname{Cab}(\overrightarrow{\mathrm{a}}, \overrightarrow{\mathrm{r}}))^{\star}, \\
\mathcal{T}(\overrightarrow{\mathrm{r}},-\overrightarrow{\mathrm{s}}) & =(\mathcal{T}(\overrightarrow{\mathrm{r}}, \overrightarrow{\mathrm{s}}))^{\star} .
\end{aligned}
$$

Changing the orientation, denoted by "-", at the $i^{\text {th }}$ step, we obtain that for any $1 \leq i \leq \ell$,

$$
\begin{aligned}
-\operatorname{Cab}(\overrightarrow{\mathrm{a}}, \overrightarrow{\mathrm{r}}) & =\operatorname{Cab}\left(\left\{\ldots, \mathrm{a}_{i-1},-\mathrm{a}_{i}, \mathrm{a}_{i+1}, \ldots\right\},\left\{\ldots, \mathrm{r}_{i-1},-\mathrm{r}_{i}, \mathrm{r}_{i+1}, \ldots\right\}\right), \\
\pm \mathcal{T}(\overrightarrow{\mathrm{r}}, \overrightarrow{\mathrm{s}}) & =\mathcal{T}\left(\left\{\ldots, \mathrm{r}_{i-1},-\mathrm{r}_{i}, \mathrm{r}_{i+1}, \ldots\right\},\left\{\ldots, \mathrm{s}_{i-1},-\mathrm{s}_{i}, \mathrm{~s}_{i+1}, \ldots\right\}\right) .
\end{aligned}
$$

We note that the Jones and HOMFLYPT polynomials for $K^{\star}$ are obtained from those for $K$ by the formal conjugation of the parameters, namely $q \mapsto q^{-1}$ and $a \mapsto a^{-1}$. This will hold for the DAHA-Jones polynomials and DAHA superpolynomials (the conjugation of $t$ will be added to that of $q$ and $a$ ). The orientation does not influence our construction.

\subsection{Algebraic knots}

There exists a deep connection of the iterated torus knots for strictly positive $r_{i}$ and $s_{i}$ with the germs of isolated plane curve singularities. Its origin is the Newton's successive approximations for $y$ in terms of $x$ satisfying a polynomial equation $f(x, y)=0$ in a neighborhood of $0=(x=0, y=0)$. The main claim is that the knot $\mathcal{T}(\vec{r}, \vec{s})$ for the characteristic pairs $\left\{\mathrm{r}_{i}, \mathrm{~s}_{i}\right\}$ with $\mathrm{r}_{i}, \mathrm{~s}_{i}>0$ is the link of the germ of the singularity

$$
y=x^{s_{1} / r_{1}}\left(c_{1}+x^{s_{2} /\left(r_{1} r_{2}\right)}\left(c_{2}+\cdots+x^{s_{\ell} /\left(r_{1} r_{2} \cdots r_{\ell}\right)}\right)\right) \quad \text { at } 0,
$$

which is the intersection of the corresponding plane curve $f(x, y)=0$ with a small 3 dimensional sphere in $\mathbb{C}^{2}$ around 0 . We will always assume that this germ is unibranch.

The inequality $\mathrm{s}_{1}<\mathrm{r}_{1}$ is commonly imposed here (otherwise $x$ and $y$ can be switched). Formula (2-10) is the celebrated Newton-Puiseux expansion, though Puiseux performed the multiplication here,

$$
y=b_{1} x^{\mathrm{m}_{1} / \mathrm{r}_{1}}+b_{2} x^{\mathrm{m}_{2} /\left(\mathrm{r}_{1} \mathrm{r}_{2}\right)}+b_{3} x^{\mathrm{m}_{3} /\left(\mathrm{r}_{1} \mathrm{r}_{2} \mathrm{r}_{3}\right)}+\cdots,
$$

for $\mathrm{m}_{1}=\mathrm{s}_{1}$ and $\mathrm{m}_{i}=\mathrm{s}_{i}+\mathrm{r}_{i} \mathrm{~m}_{i-1}$, where $\left\{\mathrm{r}_{i}, \mathrm{~m}_{i}\right\}$ are called the Puiseux pairs (which we will not use). 
We will denote the germ of the curve in (2-10) by $\mathcal{C}_{\vec{r}, \vec{s}}$. If it has more than one branch (near 0), then its complete topological description requires knowing the sequences $\{\vec{r}, \vec{s}\}$ for all components together with the pairwise linking numbers. See eg [31] and [17, page 49]. All algebraic knots can be obtained in such a way; the positivity of $r_{i}$ and $s_{i}$ is necessary for $\mathcal{T}(\vec{r}, \vec{s})$ to be an algebraic link.

Jacobian factors One can associate with a unibranch $\mathcal{C}_{\vec{r}, \vec{s}}$ the Jacobian factor $J\left(\mathcal{C}_{\vec{r}, \vec{s}}\right)$. Up to a homeomorphism, it can be introduced as the canonical compactification of the generalized Jacobian of an integral rational planar curve with $\mathcal{C}_{\overrightarrow{\mathrm{r}}, \overrightarrow{\mathrm{s}}}$ as its only singularity [29]; there is a purely local definition as well. Its dimension is the $\delta$-invariant of the singularity $\mathcal{C}_{\vec{r}, \vec{s}}$, also called its arithmetic genus.

The Jacobian factors are in the focus of many studies; see eg [4; 18, Theorem $1 ; 46$; 36; 29]. The latter paper, where the connection with the affine Springer fibers was established, provides a certain link to the theory of DAHA modules [45; 49], though we cannot relate this to our using DAHA. An important connection of $J\left(\mathcal{C}_{\vec{r}, \vec{s}}\right)$ and the rational DAHA was established in [33;23] (see references therein), which must be mentioned, but this is not connected with our approach or that from [12; 13] so far.

Calculating the Euler number e $\left(J\left(\mathcal{C}_{\vec{r}, \vec{s}}\right)\right)$, the topological Euler characteristic of $J\left(\mathcal{C}_{\vec{r}, \vec{s}}\right)$, and the corresponding Betti numbers in terms of $\vec{r}$ and $\vec{s}$ is a challenging problem. For torus knots $T(\mathrm{r}, \mathrm{s})$, one has $e\left(J\left(\mathcal{C}_{\mathrm{r}, \mathrm{s}}\right)\right)=(1 / \mathrm{r}+\mathrm{s})\left(\begin{array}{c}\mathrm{r}+\mathrm{s} \\ \mathrm{r}\end{array}\right)$ due to [4]. This formula is related to the perfect modules of rational DAHA; see eg [21].

The Euler numbers of $J\left(\mathcal{C}_{\vec{r}, \vec{s}}\right)$ were calculated in [36, Main theorem] for the following triples of Puiseux characteristic exponents:

$$
(4,2 u, v),
$$

$(6,8, v)$ and

$(6,10, v)$ for odd $u, v>0$

where $4<2 u<v, 8<v$ and $10<v$, respectively. Here, $\delta=\operatorname{dim} J\left(\mathcal{C}_{\overrightarrow{\mathrm{r}}, \overrightarrow{\mathrm{s}}}\right)$ is $(\mathrm{r}-1)(\mathrm{s}-1) / 2$ for $T(\mathrm{r}, \mathrm{s})$ and $2 u+(v-1) / 2-1$ for the series $(4,2 u, v)$. Generally, $\delta$ equals the cardinality $|\mathbb{N} \backslash \Gamma|$, where $\Gamma$ is the valuation semigroup associated with $\mathcal{C}_{\vec{r}, \vec{s}}$; see [36] and [50]. The Euler numbers of the Jacobian factors (any isolated plane curve singularities) can be also calculated now via the HOMFLYPT polynomials of the corresponding links (see below) due to $[34 ; 30]$.

Concerning the Betti numbers for the torus knots and the series $(4,2 u, v)$, the odd (co)homology of $J\left(\mathcal{C}_{\vec{r}, \vec{s}}\right)$ vanishes, and the formulas for the Betti numbers $h^{(2 k)}=$ $\operatorname{rk}\left(H^{2 k}\left(J\left(\mathcal{C}_{\vec{r}, \vec{s}}\right)\right)\right)$ are known for some values of $k$ (those sufficiently close to 0 or $\delta$ ), where $0 \leq k \leq \delta$. Not much is known/expected beyond these two series. Paper [36] contains several results and conjectures for the Betti numbers of Jacobian factors; for instance, there are quite a few formulas there for $T(\mathrm{r}, \mathrm{s})$ with $\min (\mathrm{r}, \mathrm{s}) \leq 4$. 
What DAHA provides According to Conjecture 2.4, the DAHA superpolynomial $\mathcal{H}_{\{\vec{r}, \vec{s}\}}$ (defined in Theorem 2.1) at $a=0, q=1$ in the uncolored case is $\sum_{k=0}^{\delta} h^{(2 k)} t^{k}$ for any unibranch $\mathcal{C}_{\vec{r}, \vec{s}}$, which matches the formulas from [36]. This includes/implies the van Straten-Warmt conjecture, which claims that all odd Betti numbers of $J\left(\mathcal{C}_{\vec{r}, \vec{s}}\right)$ vanish since the DAHA superpolynomials contain only integral powers of $t$. In the opposite direction, such an interpretation of $\mathcal{H}_{\{\overrightarrow{\mathrm{r}}, \vec{s}\}}(\square ; q=1, t, a=0)$ would apply its positivity. The whole (uncolored) $\mathcal{H}_{\{\vec{r}, \vec{s}\}}$ describes the geometry of Hilbert schemes of $\mathcal{C}_{\vec{r}, \vec{s}}$ due to [33, Conjecture 2].

We conjecture that the coefficients of the whole $\mathcal{H}_{\{\vec{r}, \vec{s}\}}$ are positive for any rectangle Young diagrams and algebraic knots; let us mention [6] in this respect. If such a positivity holds for one box, the corresponding knots will be called pseudoalgebraic; then $\mathcal{H}_{\{\vec{r}, \vec{s}\}}$ is expected to coincide with the uncolored stable reduced KhovanovRozansky polynomial of $\mathcal{T}(\vec{r}, \vec{s})$, producing (by definition) the Khovanov-Rozansky polynomials for $s l_{N} a=t^{N} \sqrt{q / t}$ for sufficiently large $N$. The substitution $a=-1$ here is expected to result in the Heegaard-Floer homology. This is stated in the DAHA parameters $q, t, a$; see parts (ii) and (iii) of Conjecture 2.4. We restrict ourselves there to the uncolored case, because this is needed in [33] and in the definition of the Khovanov-Rozansky triply graded homology. However, see [47] and other papers on the categorification (via quantum groups).

\subsection{DAHA-Jones polynomials}

The following theorems are mainly an extension of [13, Theorem 1.2] on DAHA-Jones polynomials from torus knots to arbitrary iterated torus knots.

Torus knots $T(\mathrm{r}, \mathrm{s})$ are naturally represented by $\gamma_{\mathrm{r}, \mathrm{s}} \in \mathrm{GL}_{2}(\mathbb{Z})$ with the first column $(r, s)^{\operatorname{tr}}$ (tr is the transposition) for $r, s \in \mathbb{Z}$ assuming that $\operatorname{gcd}(r, s)=1$. Then let $\hat{\gamma}_{\mathrm{r}, \mathrm{s}} \in \mathrm{GL}_{2}^{\wedge}(\mathbb{Z})$ be any pullback of $\gamma_{\mathrm{r}, \mathrm{s}}$.

Note that $(r, s)$ can be obviously lifted to $\gamma$ of determinant 1 and, accordingly, to $\hat{\gamma}$ from the subgroup $\operatorname{PSL}_{2}^{\wedge}(\mathbb{Z})$ generated by $\left\{\tau_{ \pm}\right\}$, ie without using $\eta$. This is actually sufficient for the construction of the DAHA-Jones polynomials below.

For a polynomial $F$ in terms of fractional powers of $q$ and $t_{\nu}$, the tilde-normalization $\widetilde{F}$ will be the result of the division of $F$ by the lowest $q, t_{v}$-monomial, assuming that it is well defined. We put $q^{\bullet} t^{\bullet}$ for a monomial factor (possibly fractional) in terms of $q$ and $t_{v}$.

Theorem 2.1 Let $R$ be a reduced irreducible root system. Recall that $H \mapsto H \Downarrow:=$ $H(1)$, where the action of $H \in \mathcal{H}$ in $\mathcal{V}$ is used. Given two sequences $\vec{r}, \vec{s}$ of length 
$\ell$ as in (2-1), we lift $\left(\mathrm{r}_{i}, \mathrm{~s}_{i}\right)^{\mathrm{tr}}$ to $\gamma_{i}$ and then to $\widehat{\gamma}_{i} \in \mathrm{GL}_{2}^{\wedge}(\mathbb{Z})$ as above. For a weight $b \in P_{+}$, the DAHA-Jones polynomial is

$$
\begin{aligned}
J D_{\overrightarrow{\mathrm{r}}, \overrightarrow{\mathrm{s}}}^{R}(b ; q, t) & =J D_{\overrightarrow{\mathrm{r}}, \overrightarrow{\mathrm{s}}}(b ; q, t) \\
& :=\left\{\widehat{\gamma}_{1}\left(\cdots\left(\widehat{\gamma}_{\ell-1}\left(\left(\widehat{\gamma}_{\ell}\left(P_{b}\right) / P_{b}\left(q^{-\rho_{k}}\right)\right) \Downarrow\right) \Downarrow\right) \cdots\right)\right\}_{e v} .
\end{aligned}
$$

It does not depend on the particular choice of the lifts $\gamma_{i} \in \mathrm{GL}_{2}(\mathbb{Z})$ and $\widehat{\gamma}_{i} \in \mathrm{GL}_{2}^{\wedge}(\mathbb{Z})$ for $1 \leq i \leq \ell$ and is indeed a polynomial in terms of $q$ and $t_{v}$. Assuming that $r_{i}, \mathrm{~s}_{i}>0$ for all $i$, ie that the $\operatorname{knot} \mathcal{T}(\overrightarrow{\mathrm{r}}, \overrightarrow{\mathrm{s}})$ is algebraic, the tilde-normalization $\widetilde{J D}_{\overrightarrow{\mathrm{r}}, \overrightarrow{\mathrm{s}}}(b ; q, t)$ is well defined and is a polynomial in terms of $q$ and $t_{v}$ with constant term 1 .

Proof The proofs of this and the next theorem almost exactly follow those in [13] (for torus knots). We will provide here only the justification of the polynomiality. If $J D_{\overrightarrow{\mathrm{r}}, \mathrm{s}}^{R}(b ; q, t)$ for $b \in P_{+}$is not a polynomial in terms of $q$ and $t_{v}$, then $P_{b}^{\circ}:=P_{b} / P_{b}\left(q^{-\rho_{k}}\right)$ has a pole at $\epsilon=0$, where $\epsilon=\left(1-q^{j} t_{\text {sht }}^{r} t_{\text {lng }}^{s}\right)$ for certain $j>0, r, s \geq 0, r+s>0$. We can assume that $\epsilon$ is a maximal such binomial, ie that there is no singularity here at $\left(1-q^{j v} t_{\text {sht }}^{r v} t_{\text {lng }}^{s v}\right) / \epsilon=0$ for any $\mathbb{Z} \ni v>1$.

Let us localize and complete the ring of coefficients of $\mathcal{H}$ and the polynomial representation $\mathcal{V}$, which is $\mathbb{Z}_{q, t}=\mathbb{Z}\left[q^{ \pm 1 /(2 \mathrm{~m})}, t_{v}^{ \pm 1 / 2}\right]$, by this $\epsilon$, ie with respect to the ideal $\left(1-q^{j} t_{\text {sht }}^{r} t_{\text {lng }}^{s}\right)$; the notations will be $\mathbb{Z}_{q, t}^{(\epsilon)}, \mathcal{H}^{(\epsilon)}$ and $\mathcal{V}^{(\epsilon)}$. Note that we added $q^{ \pm 1 /(2 \mathrm{~m})}$ to $\mathbb{Z}_{q, t}$. We will use the evaluation pairing, defined as

$$
\{E, F\}_{e v}=E\left(Y^{-1}\right)(F(X))\left(q^{-\rho_{k}}\right), \quad E, F \in \mathcal{V}^{(\epsilon)} .
$$

See [11, Theorem 11.8] and [13, Theorem 1.2]. We set $R A D_{\epsilon, \ell}=\left\{F \in \mathcal{V}^{(\epsilon)} \mid\{F, \mathcal{V}\}_{e v} \subset\right.$ $\left.\epsilon^{\ell} \mathbb{Z}_{q, t}^{(\epsilon)}\right\}$ for $\ell \in \mathbb{Z}_{+}$. Switching from $\mathcal{V}$ to the whole $\mathcal{H}$, we define

$$
R A D_{\epsilon, \ell}:=\left\{H \in \mathcal{H} \mathcal{H}^{(\epsilon)} \mid\{\mathcal{H H} H \mathcal{H} \mathcal{H}\}_{e v} \in \epsilon^{\ell} \mathbb{Z}_{q, t}^{(\epsilon)}\right\} \quad \text { for } \ell \in \mathbb{Z}_{+} .
$$

Equivalently, $R A D_{\epsilon, \ell}=\left\{H \in \mathcal{H}^{(\epsilon)} \mid H\left(\mathcal{V}^{(\epsilon)}\right) \subset \operatorname{Rad}_{\epsilon, \ell}\right\}$, since $\operatorname{Rad}_{\epsilon, \ell}=\left\{F \in \mathcal{V}^{(\epsilon)} \mid\right.$ $\left.\{\mathcal{H}(F)\}_{\text {ev }} \subset \epsilon^{\ell} \mathbb{Z}_{q, t}^{(\epsilon)}\right\}$; see [11, Lemma 11.3].

Here, $q$ is not a root of unity. Therefore, any $Y$-invariant submodule of $\mathcal{V}$ is invariant with respect to the natural action of $\tau_{-}$in $\mathcal{V}$. For instance, $\tau_{-}\left(P_{b}\right)=$ $q^{-(b, b) / 2-\left(b, \rho_{k}\right)} P_{b}$, assuming that $P_{b}$ for $b \in P_{+}$is well defined. We conclude that $\psi$ and $\tau_{-}$preserve $R A D_{\epsilon, p}$ for any $p \in \mathbb{Z}_{+}$(and generic $q$ ). The same holds for $\eta$; see (1-11). Thus, the whole $\mathrm{GL}_{2}^{\wedge}(\mathbb{Z})$ preserves each $R A D_{\epsilon, p}$.

Let $\widetilde{P}_{b}:=\epsilon^{l} P_{b}^{\circ} \in \mathcal{V}^{(\epsilon)}$ for minimal such $l \in \mathbb{N}$. Then $\widetilde{P}_{b}\left(q^{-\rho_{k}}\right) \in \epsilon^{l} \mathbb{Z}_{q, t}^{(\epsilon)}$ due to the normalization of $P_{b}^{\circ}$. Since $\widetilde{P}_{b}$ is an eigenfunction of $\left\{L_{f}\right\}$ from (1-18), one has that $\widetilde{P}_{b} \in \operatorname{Rad}_{\epsilon, l}$; see [11, Lemmas 11.4-5]. 
This implies that $\hat{\gamma}\left(\widetilde{P}_{b}\right) \in R A D_{\epsilon, l}$ for any $\gamma \in \operatorname{PSL}_{2}(\mathbb{Z})$. The projection $\widetilde{P}^{\prime}=\widehat{\gamma}\left(\widetilde{P}_{b}\right) \Downarrow$ $=\hat{\gamma}\left(\widetilde{P}_{b}\right)(1)$ then belongs to $\operatorname{Rad}_{\epsilon, l}$. Hence, $\hat{\gamma}^{\prime}\left(\widetilde{P}^{\prime}\right) \in R A D_{\epsilon, l}$ for any $\gamma^{\prime} \in \operatorname{PSL}_{2}(\mathbb{Z})$ and we can continue. One automatically obtains that $\left\{\widetilde{P}^{\prime \prime}\right\}_{e v}$ is divisible by $\epsilon^{l}$ for $\widetilde{P}^{\prime \prime}:=\widehat{\gamma}^{\prime}\left(\widetilde{P}^{\prime}\right) \Downarrow$ and for any further such polynomials obtained by this procedure for $\gamma^{\prime \prime}, \gamma^{\prime \prime \prime}$, and so on.

We conclude that $J D_{\overrightarrow{\mathrm{r}}, \overrightarrow{\mathrm{s}}}(b ; q, t)$ has actually no singularity at $\epsilon=0$, which is a contradiction sufficient to claim polynomiality of $J D_{\overrightarrow{\mathrm{r}}, \overrightarrow{\mathrm{s}}}(b ; q, t)$ for any $b \in P_{+}, \vec{r}$ and $\overrightarrow{\mathrm{s}}$. We mention that the nonsymmetric Macdonald polynomials are used in the corresponding reasoning from [13], which are actually more convenient here than $P_{b}$.

Theorem 2.2 (i) The polynomials $J D_{\overrightarrow{\mathrm{r}}, \overrightarrow{\mathrm{s}}}(b ; q, t)$ coincide up to $q^{\bullet} t^{\bullet}$ for the pairs from 2.1-2.1, ie for $\{\vec{r}, \vec{s}\}$ with isotopic $\mathcal{T}(\vec{r}, \vec{s})$. Also, (2-7) corresponds to

$$
J D_{\overrightarrow{\mathrm{r}}, \overrightarrow{\mathrm{s}}}(b ; q, t)=J D_{\left\{\mathrm{s}_{1}, \mathrm{r}_{2}, \ldots, \mathrm{r}_{\ell}\right\},\left\{\mathrm{r}_{1}, \mathrm{~s}_{2}, \ldots, \mathrm{s}_{\ell}\right\}}(b ; q, t),
$$

and the following DAHA counterparts of (2-8) and (2-9) hold:

$$
\begin{aligned}
& J D_{\overrightarrow{\mathrm{r}},-\overrightarrow{\mathrm{s}}}(b ; q, t)=\left(J D_{\overrightarrow{\mathrm{r}}, \overrightarrow{\mathrm{s}}}(b ; q, t)\right)^{\star} \quad \text { for } \star \text { from }(1-14), \\
& J D_{\overrightarrow{\mathrm{r}}, \overrightarrow{\mathrm{s}}}=J D_{\left\{\ldots, \mathrm{r}_{i-1},-\mathrm{r}_{i}, \mathrm{r}_{i+1}, \ldots\right\},\left\{\ldots, \mathrm{s}_{i-1},-\mathrm{s}_{i}, \mathrm{~s}_{i+1}, \ldots\right\}} \quad \text { for } 1 \leq i \leq \ell .
\end{aligned}
$$

(ii) Let us assume that, for $b, c \in P_{+}$and certain $w \in W$ with $w \neq \mathrm{id}$,

$$
q^{\left(b+\rho_{k}-w\left(\rho_{k}\right)-w(c), \alpha\right)}=1=q_{\alpha}^{\left(b-w(c), \alpha^{\vee}\right)} t_{\text {sht }}^{\left(\rho_{\text {sht }}^{w}, \alpha^{\vee}\right)} t_{\operatorname{lng}}^{\left(\rho_{\operatorname{lng}}^{w}, \alpha^{\vee}\right)}
$$

for any $\alpha \in R_{+}$, where we set $\rho_{v}^{w}:=w\left(\rho_{v}\right)-\rho_{v}$. Then

$$
J D_{\overrightarrow{\mathrm{r}}, \overrightarrow{\mathrm{s}}}(b ; q, t)=J D_{\overrightarrow{\mathrm{r}}, \overrightarrow{\mathrm{s}}}(c ; q, t) \quad \text { for such } q,\left\{t_{v}\right\}
$$

and for any $\vec{r}$ and $\vec{s}$. Also, for $b=\sum_{i=1}^{n} b_{i} \omega_{i}$,

$$
J D_{\overrightarrow{\mathrm{r}}, \overrightarrow{\mathrm{s}}}(b ; q=1, t)=\prod_{i=1}^{n} J D_{\overrightarrow{\mathrm{r}}, \overrightarrow{\mathrm{s}}}\left(\omega_{i} ; q=1, t\right)^{b_{i}} \quad \text { for any } \overrightarrow{\mathrm{r}} \text { and } \overrightarrow{\mathrm{s}} \text {. }
$$

Justifying the symmetries from part (i) is essentially parallel to those in [13, Theorem 1.2]. Let us comment on 2.1. Essentially, one needs to check here that the torus knot $T(m r+\mathrm{s}, \mathrm{r})$ results in the same DAHA-Jones polynomial as the " 2 -cable" corresponding to the Newton pairs $\{1, m\},\{r, s\}$. Topologically, $T(m r+\mathrm{s}, \mathrm{r})$ is isotopic to $C a b(m r+\mathrm{s}, \mathrm{r}) T(m, 1)$, since $T(m, 1)$ is the unknot. Algebraically, such a coincidence is an interesting DAHA calculation.

Extending the connection conjectures from [12], we expect that $J D_{\vec{r}, \vec{s}}\left(b ; q, t_{v} \mapsto q_{\nu}\right)$ coincide up to $q^{\bullet}$ with the reduced quantum group (WRT) invariants for the corresponding $\mathcal{T}(\vec{r}, \vec{s})$ and any $b \in P_{+}$. The quantum group is associated with the root 
system $\widetilde{R}$; see [12]. The shift operator was used there to deduce this coincidence from [43] in the case of $A_{n}$ and torus knots; quite a few confirmations were provided for other root systems (including special ones).

Using the shift operator for the iterated knots is almost exactly the same as for the torus knots. The QG invariants of iterated knots are less studied than those for the torus knots, though the Rosso-Jones formula can be potentially used for them and any root systems. In the case of $A_{1}$, we will prove (i) using the formulas from [38; 32].

\subsection{DAHA superpolynomials}

Following [12; 22; 13], Theorems 2.1 and 2.2 can be extended to the DAHA superpolynomials, which includes the stabilization of $J D^{A_{n}}(b ; q, t)$ with respect to $n$. This stabilization was announced in [12]; its proof was published in [22]. Both approaches use [41] and can be extended to arbitrary iterated knots; the DAHA duality conjecture proposed in [12] was proven in [22].

We mainly omit the justifications in this paper; with few reservations, they are similar to those in $[22 ; 13]$ (for torus knots). The focus of this paper is on the connections with the Khovanov-Rozansky theory and the geometry of (germs of) unibranch plane curve singularities.

The sequences $\vec{r}$ and $\vec{s}$ will be as above ( $\ell$ is the length), as well as the iterated knots $\mathcal{T}(\vec{r}, \vec{s})$. We will also use the DAHA-Jones polynomials $\widetilde{J D}_{\overrightarrow{\mathrm{r}}, \overrightarrow{\mathrm{s}}}$ and (later) the Jacobian factors $J\left(\mathcal{C}_{\vec{r}, \vec{s}}\right)$ of $\mathcal{C}_{\vec{r}, \vec{s}}$, which are the germs of unibranch isolated plane curve singularities; see Section 2.2.

Theorem 2.3 We switch to the root system $A_{n}$ for $s l_{n+1}$, setting $t=t_{\text {sht }}=q^{k}$. Let us consider $P_{+} \ni b=\sum_{i=1}^{n} b_{i} \omega_{i}$ as a (dominant) weight for any $A_{m}$ (for $s l_{m+1}$ ) with $m \geq n-1$, where we set $\omega_{n}=0$ upon the restriction to $A_{n-1}$.

(i) Stabilization Given $\mathcal{T}(\overrightarrow{\mathrm{r}}, \overrightarrow{\mathrm{s}})$, there exists $\mathcal{H}_{\overrightarrow{\mathrm{r}}, \overrightarrow{\mathrm{s}}}(b ; q, t, a)$, a polynomial from $\mathbb{Z}\left[q, t^{ \pm 1}, a\right]$, satisfying the relations

$$
\mathcal{H}_{\overrightarrow{\mathrm{r}}, \overrightarrow{\mathrm{s}}}\left(b ; q, t, a=-t^{m+1}\right)= \pm q^{\bullet} t^{\bullet} J D_{\overrightarrow{\mathrm{r}}, \overrightarrow{\mathrm{s}}}^{A m}(b ; q, t) \quad \text { for any } m \geq n-1,
$$

and normalized as follows. The polynomial $\mathcal{H}(a=0)$ is assumed minimal, ie not divisible by $q, t$ or any prime number; the coefficient of the minimal power of $t$ in $\mathcal{H}(q=0, a=0)$ is selected positive.

(ii) Symmetries The corresponding normalization of JD-polynomials, denoted by $\widetilde{J D}$, extends the tilde-normalization for algebraic knots from Theorem 2.1. For sufficiently large $m$ (for all $m \geq n-1$ if $\mathrm{r}_{i}, \mathrm{~s}_{i}>0$ ),

$$
\mathcal{H}_{\overrightarrow{\mathrm{r}}, \overrightarrow{\mathrm{s}}}\left(b ; q, t, a=-t^{m+1}\right)=\widetilde{J D}_{\overrightarrow{\mathrm{r}}, \overrightarrow{\mathrm{s}}}^{A m}(b ; q, t) .
$$


The symmetries from part (i) of Theorem 2.2 hold for $\mathcal{H}_{\vec{r}, \vec{s}}$, except for (2-15); the latter holds for $a^{\star}=a^{-1}$ and up to $a^{\bullet} q^{\bullet} t^{\bullet}$.

(iii) Color exchange Imposing (2-16), we consider $w$ as an element of $\mathbf{S}_{m+1}$ (the Weyl group for $A_{m}$ ) for every $m \geq n$, naturally acting in the corresponding $P$. Then for any $\vec{r}$ and $\vec{s}$,

$$
\mathcal{H}_{\overrightarrow{\mathrm{r}}, \overrightarrow{\mathrm{s}}}(b ; q, t, a)=\mathcal{H}_{\overrightarrow{\mathrm{r}}, \overrightarrow{\mathrm{s}}}(c ; q, t, a) \quad \text { for such } q, t .
$$

In particular, let $w=s_{i}=(i, i+1)$ with $i<n$ and $b=c-\left(k+\left(c, \alpha_{i}\right)\right) \alpha_{i}$ for a dominant $c$. Then the inequalities $c_{i} / 2 \leq-k \leq c_{i}+\min \left\{c_{i \pm 1>0}\right\}$ for $k \in-\mathbb{Z}_{+}$ imply (2-16).

(iv) Specialization and $\operatorname{deg}_{a} \quad$ Making $q=1$, one has

$$
\mathcal{H}_{\overrightarrow{\mathrm{r}}, \overrightarrow{\mathrm{s}}}(b ; q=1, t, a)=\prod_{i=1}^{n} \mathcal{H}_{\overrightarrow{\mathrm{r}}, \overrightarrow{\mathrm{s}}}\left(\omega_{i} ; q=1, t, a\right)^{b_{i}} \quad \text { for } b=\sum_{i=1}^{n} b_{i} \omega_{i}
$$

Assuming that $\left|\mathrm{r}_{1}\right|>\left|\mathrm{s}_{1}\right|$ and $\mathrm{r}_{i} \neq 0$ for $j>1, \operatorname{deg}_{a} \mathcal{H}_{\overrightarrow{\mathrm{r}}, \overrightarrow{\mathrm{s}}}(b ; q, t, a)$ (the $a-$ degree) is no greater than $\left(\max \left\{1,\left|s_{1}\right|\right\}\left|r_{2} \cdots r_{\ell}\right|-1\right)$ times $\operatorname{ord}\left(\lambda_{b}\right)$, the number of boxes in the Young diagram $\lambda_{b}$ associated with $b \in P_{+}$.

(v) Superduality $[25 ; 12 ; 22] U p$ to a power of $q$ and $t$,

$$
\mathcal{H}_{\overrightarrow{\mathrm{r}}, \overrightarrow{\mathrm{s}}}(\lambda ; q, t, a)=q^{\bullet} t^{\bullet} \mathcal{H}_{\overrightarrow{\mathrm{r}}, \overrightarrow{\mathrm{s}}}\left(\lambda^{\mathrm{tr}} ; t^{-1}, q^{-1}, a\right),
$$

where we switch from (arbitrary) dominant weights $b$ to the corresponding Young diagrams $\lambda=\lambda_{b}$, and $\lambda^{\text {tr }}$ is the transposition of $\lambda$.

Concerning the stabilization and duality, we mainly follow here [22], switching from $P_{\lambda}$ to the modified Macdonald polynomials. Since the projective action of $\operatorname{PSL}(2, \mathbb{Z})$ is compatible with such a passage (see $[22 ; 41]$ ), the transition to the cables of torus knots is relatively direct (with modest deviations for nonalgebraic knots). Note that the DAHA of type $g l_{n+1}$ and that for $s l_{n+1}$ (of type $A_{n}$ ) result in coinciding DAHA-Jones polynomials up to $q^{\bullet} t^{\bullet}$, which is not too difficult to justify. The compatibility of DAHA and the Macdonald polynomials of types $g l_{n+1}$ and $s l_{n+1}$ is used here.

Color exchange See [13] for a systematic consideration of the color exchange in (2-21). In terms of the Young diagrams it is as follows. We associate with $c=$ $\sum_{i=1}^{n} c_{i} \omega_{i}$ in part (iii) the Young diagram

$$
\lambda_{c}=\left\{m_{1}=c_{1}+\cdots+c_{n}, m_{2}=c_{1}+\cdots+c_{n-1}, \ldots, m_{n}=c_{n}, 0,0, \ldots\right\} .
$$

Then, we switch to $\lambda_{c}^{\prime}=\left\{m_{i}^{\prime}=m_{i}-k(i-1)\right\}$, apply $w \in W$ to $\lambda_{c}^{\prime}$, and finally obtain

$$
\lambda_{b}=\left\{m_{w(i)}^{\prime}+k(i-1)\right\}=\left\{m_{w(i)}+k(i-w(i))\right\} .
$$


Here, $w$ transforms the rows of $\lambda$, and we set

$$
w\left\{m_{1}, m_{2}, \ldots, m_{n}\right\}=\left\{m_{w(1)}, m_{w(2)}, \ldots, m_{w(n)}\right\} .
$$

Given $k<0$ (it can be fractional), $\lambda_{b}$ must be a Young diagram; this condition determines which $w$ can be used.

Toward $C^{\vee} C$ The second part of [12] is devoted to the DAHA-Jones polynomials for the root system $C^{\vee} C_{1}$, which prepared the extension of the DAHA-Jones theory to all $C^{\vee} C_{n}$. Furthermore, we expect here a relatively smooth switch to arbitrary iterated knots. There is an interesting application of DAHA-Jones polynomials for $C^{\vee} C_{1}$ to the superpolynomials of type $A$ for knots $T(2 \mathrm{~m}+1,2)$ (ie any $A_{n}$ ). We see relations of this kind for certain iterated knots, say $\operatorname{Cab}(2 \mathrm{~m}+1,2) T(3,2)$, but we do not know how far this can go; cf [16].

The $a$-degree Due to (2-22), it suffices to check the equality for the fundamental weights only. We expect that, for algebraic knots, $\operatorname{deg}_{a} \mathcal{H}_{\overrightarrow{\mathrm{r}}, \overrightarrow{\mathrm{s}}}(b ; q, t, a)$ from part (iv) equals $\left(s_{1} r_{2} \cdots r_{\ell}-1\right) \operatorname{ord}\left(\lambda_{b}\right)$ (there is no such coincidence in general). This equality is likely to hold for the HOMFLYPT polynomials of algebraic knots (the inequality from part (iv) holds for all iterated knots), which would formally result in such an equality for $\mathcal{H}$ assuming part (i) of Conjecture 2.4 below.

\subsection{Connection conjecture}

Let us briefly discuss the HOMFLYPT and Khovanov-Rozansky polynomials. The stable Khovanov-Rozansky homology is the $s l_{N}$ homology from [27; 28] in the range of $N$ where the isomorphism in [37, Theorem 1] holds; see also [26]. Thus, they can be recovered from the triply graded HOMFLYPT homology. The cabling formula for the quantum group invariants can be found in [32, Main theorem]; it provides the formulas for the HOMFLYPT polynomials of torus iterated knots. See also [3; 2] and [30] for the HOMFLYPT skein relations.

We need to restrict part (ii) of the conjecture below to the uncolored case, since the Khovanov-Rozansky triply graded homology requires this. The corresponding reduced Poincaré polynomial, the so called stable Khovanov-Rozansky polynomial, is denoted by $\operatorname{KhR}_{\text {stab }}(q, t, a)$ in what will follow. The passage to the stable Khovanov-Rozansky polynomials for $s l_{N}$, denoted by $K h R_{N}$, is $a \mapsto t^{N} \sqrt{q / t}$. Equivalently, $a_{s t} \mapsto q_{s t}^{N}$ in the standard parameters from the conjecture below. We always consider them under the tilde-normalization; the notation will be $\widetilde{K h R}_{N}$. Note the relation to the Heegaard-Floer homology for $N=0$. 
We mention that categorification theory can generally deal with arbitrary weights, but this is not sufficiently understood in such a generality. See eg [26; 48; 39; 47] and references therein. Also, our construction is reduced (the normalization is by 1 at $\bigcirc$, necessary for the polynomiality of DAHA-Jones polynomials), which creates additional difficulties for the categorification.

The polynomials $K h R_{\text {stab }}$ are expected to coincide with the (reduced) physics superpolynomials based on the BPS states $[15 ; 1 ; 19 ; 20]$ (their theory is not mathematically rigorous) and those obtained in terms of rational DAHA $[23 ; 22]$ for torus knots. The latter are known only in the uncolored case so far; the case of symmetric powers of the fundamental representation is in progress, see [20].

We note that using the Macdonald polynomials instead of Schur functions in the construction of the so called knot operators was suggested in [1], which triggered [12]. These operators naturally appear in the approach to the Jones and WRT invariants of torus knots via the Verlinde algebras. This method was actually justified mathematically only for the root systems $A$ and $D$. It requires roots of unity $q$ and the formula for the Verlinde $S$-operator, which becomes very involved in the Macdonald setting (even for $A_{1}$ ). Also, the final output must be uniform in terms of $q$, which cannot be really explained in this approach; this seems the main reason why only the simplest superpolynomials were discussed in [1]. These problems were resolved (actually bypassed) in [12] for any reduced root systems and dominant weights.

Conjecture 2.4 (Connection conjecture) Let $\mathcal{H}_{\vec{r}, \vec{s}}(b ; q, t, a)_{s t}$ denote $\mathcal{H}_{\overrightarrow{\mathrm{r}}, \overrightarrow{\mathrm{s}}}(b ; q, t, a)$ expressed in terms of the standard parameters (in the Khovanov-Rozansky theory; see [12] and [33, Section 1]):

$$
\begin{aligned}
& t=q_{s t}^{2}, \quad q=\left(q_{s t} t_{s t}\right)^{2}, \quad a=a_{s t}^{2} t_{s t}, \\
& q_{s t}^{2}=t, \quad t_{s t}=\sqrt{q / t}, \quad a_{s t}^{2}=a \sqrt{t / q} .
\end{aligned}
$$

(i) We conjecture that for any $\vec{r}$ and $\vec{s}$ as above (possibly negative),

$$
\mathcal{H}_{\overrightarrow{\mathrm{r}}, \overrightarrow{\mathrm{s}}}(b ; q, t \mapsto q, a \mapsto-a)=\widetilde{H O M}_{\overrightarrow{\mathrm{r}}, \overrightarrow{\mathrm{s}}}\left(\lambda_{b} ; q, a\right),
$$

where the latter is the tilde-normalization of the (reduced) HOMFLYPT polynomial; $b$ is an arbitrary dominant weight represented by the Young diagram $\lambda_{b}$. The authors conjecture that the $a$-degrees of these polynomials are equal to $\left(s_{1} r_{2} \cdots r_{\ell}-1\right) \operatorname{ord}\left(\lambda_{b}\right)$ provided the positivity of $s_{i}$ and $r_{i}$, and that $s_{1}<r_{1}$.

(ii) For uncolored pseudoalgebraic knots (those with positive coefficients of uncolored DAHA superpolynomials), one has

$$
\mathcal{H}_{\overrightarrow{\mathrm{r}}, \overrightarrow{\mathrm{s}}}(\square ; q, t, a)_{s t}=\widetilde{K h R}_{\text {stab }}\left(q_{s t}, t_{s t}, a_{s t}\right), \quad \text { where } \square=\omega_{1} .
$$


We denote by $\widetilde{K h R_{\text {stab }}}$ the (reduced) $K h R_{\text {stab }}$ divided by the smallest power of $a_{s t}$ and by $q_{s t}^{\bullet} t_{\text {st }}^{\bullet}$ such that $\widetilde{K h R}_{\text {stab }}\left(a_{s t}=0\right) \in \mathbb{Z}_{+}\left[q_{s t}, t_{s t}\right]$ with the constant term 1 .

(iii) Following Section 2.2, let $\mathcal{C}_{\vec{r}, \vec{s}}$ be the (unibranch) germ at 0 of the plain curve singularity corresponding to $\left\{\mathrm{r}_{i}, \mathrm{~s}_{i}>0\right\}$ and $h^{(k)}=\operatorname{rk}\left(H^{k}\left(J\left(\mathcal{C}_{\vec{r}, \overrightarrow{\mathrm{s}}}\right)\right)\right)$ the Betti numbers of the Jacobian factor $J\left(\mathcal{C}_{\vec{r}, \vec{s}}\right)$. Then [33, Conjecture 2] and claim (ii) above result in the coincidence of the tilde-normalization of reduced $\mathscr{P}_{\text {alg }}$ there with $\mathcal{H}_{\vec{r}, \vec{s}}(\square ; q, t, a)_{s t}$; see formula (4-24) below for some details. We also conjecture that

$$
\mathcal{H}_{\overrightarrow{\mathrm{r}}, \overrightarrow{\mathrm{s}}}(\square ; q=1, t, a=0)=\sum_{i=0}^{2 \delta} h^{(i)} t^{i / 2} \quad \text { for } \delta=\operatorname{dim} J\left(\mathcal{C}_{\overrightarrow{\mathrm{r}}, \overrightarrow{\mathrm{s}}}\right),
$$

which implies that $h^{\text {odd }}=0$ (the van Straten-Warmt conjecture).

We hope that we will be able to justify part (i) in the Connection conjecture following [12, Proposition 2.3], where we used [43] (for torus knots), and Proposition 4.2 below, which states that

$$
\mathcal{H}\left(m \omega_{1} ; q, q,-q^{2}\right)=\widetilde{H O M}\left(m \omega_{1} ; q, q^{2}\right)=\widetilde{J D}^{A_{1}}\left(m \omega_{1} ; q, q\right) .
$$

The latter is the reduced tilde-normalized Jones polynomial for any iterated knot $\mathcal{T}(\vec{r}, \vec{s})$. Using [38; 32], this approach can be generally extended to any root systems.

Concerning part (ii), it is generally difficult to calculate $K h R_{n+1}$ except for $n=1$ (the celebrated Khovanov polynomials), especially for cables; $K h R_{\text {stab }}$ are of a more algebraic nature, but not too much simpler. Even if $K h R_{\text {stab }}$ is known, the problem of recovering all individual $K h R_{n+1}$ from it is quite a challenge. This is generally provided by the theory of differentials $\partial_{n+1}$ from [26; 37]. The corresponding homology associated with $\partial_{n+1}$ gives $K h R_{n+1}$ for any $n \geq 1$.

These differentials are involved, but their certain algebraic simplification, suggested in [37; 15] and developed further in [12], works surprisingly well for sufficiently small knots. The assumption in [12] is that the actual $\partial_{n+1}$ are "as surjective as possible" beginning with $a=0$. See [12, Conjecture 2.7; Section 3.6]; the "smallest" torus knot when $K h R_{2}$ cannot be obtained this way is $T(12,7)$. The output of this reduction procedure will be denoted by $D A H A^{\prime} K h R_{n+1}$.

For example, if $\mathcal{H}$ (in the DAHA-parameters) contains $q^{i}\left(t^{j} a^{m}+t^{j-n-1} a^{m+1}+\right.$ $t^{j-2 n-2} a^{m+2}$, where $q^{i} t^{i} a^{m}$ was not involved in the reductions for smaller $\operatorname{deg}_{a}$, then $q^{i} t^{j-2 n-2} a^{m+2}$ will go to $D A H A^{\prime} K h R_{n+1}$ from this triple (subject to possible further reductions). However if the actual (topological) $\partial_{n+1}$ is not onto when acting from the space associated with $q^{i} t^{j-n-1} a^{m+1}$ to that for $q^{i} t^{j} a^{m}$, then $q^{i} t^{j} a^{m}$ is the 
right term to pick for $K h R_{n+1}$. Thus, there can be topological corrections, which makes the above recovery algorithm insufficient. Nevertheless, even if these corrections are present, one can expect that $\widetilde{K h R}_{2}-D A H A^{\prime} K h R_{2}$ is a linear combination of $q_{s t}^{i} t_{s t}^{j}\left(1+t_{s t}\right)$ (the case of $\left.\partial_{2}=0\right), q_{s t}^{i} t_{s t}^{j}\left(1-t_{s t}^{2}\right)$ and so on with nonnegative coefficients. If this is the case, then such a positivity can be considered a confirmation of part (ii), which always holds in the examples of pseudoalgebraic knots we considered.

We extend this procedure to arbitrary iterated knots changing all " -1 " by $t^{n+1} / a$ in $\mathcal{H}(\square ; q, t, a)$ followed by the reduction as above. Practically, one deletes in $\mathcal{H}$ all pairs $\pm t^{i} q^{j} a^{m}\left(t^{n+1}+a\right)$ and $t^{i} q^{j} a^{m}\left(t^{2 n+2}-a^{2}\right)$ beginning with $m=0$, then switches to the standard parameters and substitutes $-1 \mapsto 1 / t_{s t}$. We will also use $D A H A^{+} K h R$, where -1 is replaced by $a / t^{n+1}$ before applying the reduction procedure. In all (uncolored) examples we considered, one of these two procedures gives correct $\widetilde{K h R}_{n+1}$ for stable $n$, or else results in the corrections as above for any $n$. Presumably $D A H A^{+} K h R$ must be used here when the $a$-leading term of $\mathcal{H}(\square ; q, t, a)$ is positive, but the evidence is limited so far.

\section{Numerical confirmations}

We will consider examples (mainly numerical) confirming the Connection conjecture. We selected only really necessary, relatively simple and instructional ones. They are expected to contain a lot of geometric-topological information (including the Betti numbers of Jacobian factors), and are important confirmations of our Connection conjecture. We do not discuss torus knots here; see [12; 13].

\subsection{Simplest algebraic iterations}

$$
\overrightarrow{\mathrm{r}}=\{3,2\}, \quad \overrightarrow{\mathrm{s}}=\{2,1\}, \quad \mathcal{T}=\operatorname{Cab}(13,2) T(3,2) ;
$$

$\mathcal{H}_{\overrightarrow{\mathrm{r}}, \overrightarrow{\mathrm{s}}}(\square ; q, t, a)=1+q t+q^{2} t+q^{3} t+q^{2} t^{2}+q^{3} t^{2}+2 q^{4} t^{2}+q^{3} t^{3}+q^{4} t^{3}+2 q^{5} t^{3}$

$$
\begin{array}{r}
+q^{4} t^{4}+q^{5} t^{4}+2 q^{6} t^{4}+q^{5} t^{5}+q^{6} t^{5}+q^{7} t^{5}+q^{6} t^{6}+q^{7} t^{6}+q^{7} t^{7}+q^{8} t^{8} \\
+a^{3}\left(q^{6}+q^{7} t+q^{8} t^{2}\right) \\
+a^{2}\left(q^{3}+q^{4}+q^{5}+q^{4} t+2 q^{5} t+2 q^{6} t+q^{5} t^{2}+2 q^{6} t^{2}+2 q^{7} t^{2}\right. \\
\left.+q^{6} t^{3}+2 q^{7} t^{3}+q^{8} t^{3}+q^{7} t^{4}+q^{8} t^{4}+q^{8} t^{5}\right) \\
+a\left(q+q^{2}+q^{3}+q^{2} t+2 q^{3} t+3 q^{4} t+q^{5} t+q^{3} t^{2}+2 q^{4} t^{2}+4 q^{5} t^{2}\right. \\
+q^{6} t^{2}+q^{4} t^{3}+2 q^{5} t^{3}+4 q^{6} t^{3}+q^{7} t^{3}+q^{5} t^{4}+2 q^{6} t^{4} \\
\left.+3 q^{7} t^{4}+q^{6} t^{5}+2 q^{7} t^{5}+q^{8} t^{5}+q^{7} t^{6}+q^{8} t^{6}+q^{8} t^{7}\right) .
\end{array}
$$


Betti numbers We will systematically provide the values of the DAHA superpolynomials at $a=0, q=1$; the corresponding Betti numbers (conjecturally coinciding with their $t$-coefficients) are known only in 2 cases from the eight uncolored algebraic knots we consider. We will also give the formula for such a DAHA-Betti polynomial for $2 \omega_{1}$ and $\mathcal{T}_{\{3,2\},\{2,1\}}=\operatorname{Cab}(13,2) T(3,2)$, which is the square of $\mathcal{H}(q=1, t, a=0)$ in (3-1), that for $\omega_{2}$, and calculate the DAHA-Betti polynomials for nonalgebraic knots. As for the latter, the coefficients in (3-11) and (3-12) are clear predecessors of the Betti numbers for $\operatorname{Cab}(13,2) T(3,2)$, the first algebraic knot in the family $C a b(2 m+1,2) T(3,2)$ for $\mathrm{m} \in \mathbb{Z}$.

For $\vec{r}=\{3,2\}, \vec{s}=\{2,1\}$, the DAHA-Betti polynomial equals

$$
\mathcal{H}(q=1, t, a=0)=1+3 t+4 t^{2}+4 t^{3}+4 t^{4}+3 t^{5}+2 t^{6}+t^{7}+t^{8},
$$

which gives exactly the first line of Betti numbers from the table before [36, Conjecture 23]; the corresponding Puiseux exponents are $(4,6,7)$. The next example gives the second line in this table, for the exponents $(4,6,9)$; see formula (3-2) below. The corresponding two $\mathcal{C}_{\vec{r}, \vec{s}}$ are represented by the local rings $\mathcal{R}=\mathbb{C} \llbracket z^{4}, z^{6}+z^{7,9} \rrbracket$; $\delta=8,9$. Note that the arithmetic genus $\delta$ always coincides with the $t$-degree of the Betti polynomial (in the examples below). Any other $\mathcal{H}(q=1, t, a=0)$ provided below are beyond known (or conjectured) formulas for the Betti numbers of $J\left(\mathcal{C}_{\vec{r}, \vec{s}}\right)$ (as far as we know).

HOMFLYPT polynomial It coincides with the corresponding DAHA-generated tildenormalized HOMFLYPT polynomial:

$$
\begin{array}{r}
\mathcal{H}(q, t \mapsto q, a \mapsto-a) \\
=1+q^{2}+q^{3}+2 q^{4}+q^{5}+3 q^{6}+q^{7}+3 q^{8}+q^{9}+3 q^{10}+q^{11}+2 q^{12}+q^{13}+q^{14}+q^{16} \\
-a^{3}\left(q^{6}+q^{8}+q^{10}\right) \\
+a^{2}\left(q^{3}+q^{4}+2 q^{5}+2 q^{6}+3 q^{7}+2 q^{8}+3 q^{9}+2 q^{10}+2 q^{11}+q^{12}+q^{13}\right) \\
-a\left(q+q^{2}+2 q^{3}+2 q^{4}+4 q^{5}+3 q^{6}+5 q^{7}+3 q^{8}+5 q^{9}\right. \\
\left.+3 q^{10}+4 q^{11}+2 q^{12}+2 q^{13}+q^{14}+q^{15}\right) .
\end{array}
$$

Up to $q^{\bullet}$, this polynomial is equal to the one considered in [34, Section 7] upon its division by $a^{16}$ and the substitution $z=q-q^{-1}$. We need to change $a^{2}$ and $q^{2}$ there by our $a$ and $q$, ie switch from the standard parameters to ours.

All superpolynomials we obtained (we provide here only some), including nonalgebraic iterated knots, satisfy the HOMFLYPT part of the Connection conjecture. The HOMFLYPT formulas will be omitted below. We used software by S Artamonov for calculating colored HOMFLYPT polynomials, connected with papers [3; 2]. 


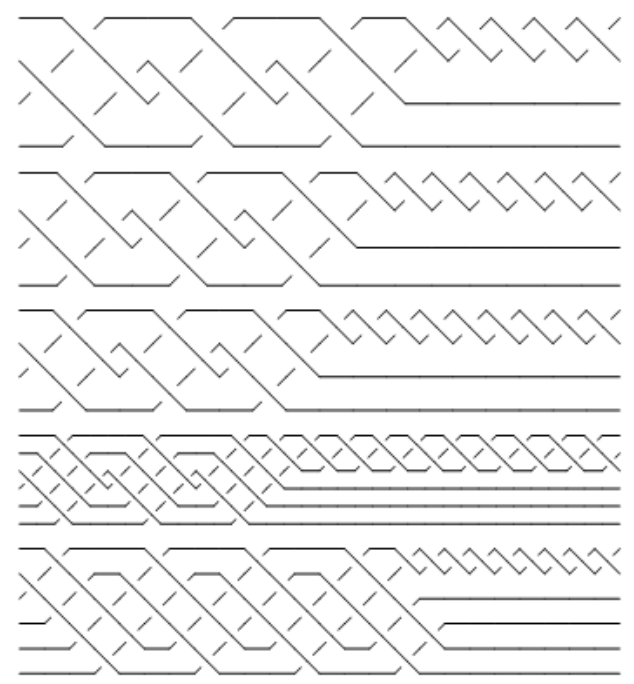

Figure 1: Cables $(11,2),(13,2),(15,2)$ of $T(3,2), \operatorname{Cab}(19,3) T(3,2)$ and $\operatorname{Cab}(25,2) T(4,3)$.

Figure 1 contains the braid for the knot $\operatorname{Cab}(13,2) T(3,2)$ and further iterated knots we will consider below. These presentations are used to calculate the HOMFLYPT polynomials and Khovanov polynomials.

Khovanov-Rozansky polynomials We provide the corresponding DAHA-Khovanov polynomials for all examples below in the standard parameters; for instance, $q$ and $t$ in the next two formulas are $q_{s t}$ and $t_{s t}$ from the conjecture. If the algorithm from [12] results in $D A H A^{\prime} K h:=D A H A^{\prime} K h R_{2}$ matching the Khovanov polynomial (for $s l_{2}$ ), then this is an indication that $D A H A^{\prime} K h R_{n+1}$ can match $\widetilde{K h R}_{n+1}$ for all $n$.

For this and the next knot, we will provide $D A H A^{\prime} K h R_{3}$, but will omit them in all other examples (they were calculated for any $n$ ); $\widetilde{K h R}_{\geq 3}$ are unknown for the knots we consider here.

(1) $D A H A^{\prime} K h(C a b(13,2) T(3,2))=1+q^{4} t^{2}+q^{6} t^{3}+q^{6} t^{4}+q^{10} t^{5}+q^{8} t^{6}$

$$
+q^{12} t^{7}+2 q^{12} t^{8}+2 q^{14} t^{9}+q^{16} t^{10}+2 q^{18} t^{11}+2 q^{20} t^{12}+q^{22} t^{13},
$$

$D{ }^{\prime} H A^{\prime} K_{h R}(\operatorname{Cab}(13,2) T(3,2))=1+q^{4} t^{2}+q^{8} t^{3}+q^{6} t^{4}+q^{8} t^{4}+q^{10} t^{5}$

$$
\begin{aligned}
& +q^{12} t^{5}+q^{8} t^{6}+q^{10} t^{6}+2 q^{14} t^{7}+q^{16} t^{7}+2 q^{12} t^{8}+q^{14} t^{8}+q^{18} t^{8} \\
+ & 2 q^{16} t^{9}+2 q^{18} t^{9}+2 q^{16} t^{10}+q^{22} t^{10}+3 q^{20} t^{11}+q^{22} t^{11}+2 q^{20} t^{12} \\
+ & q^{24} t^{12}+q^{26} t^{12}+3 q^{24} t^{13}+q^{24} t^{14}+q^{28} t^{14}+2 q^{28} t^{15}+q^{32} t^{16}
\end{aligned}
$$


This knot, the next one, and the last two knots ( 6 and 7 below) have their DAHAKhovanov polynomials $D A H A^{\prime} K h(n=2)$ coinciding with the actual Khovanov polynomials (under the tilde-normalization).

$\mathcal{H}_{\{\mathbf{3}, \mathbf{2}\},\{\mathbf{2}, \mathbf{3}\}}$ switch from $\operatorname{Cab}(13,2)$ to the "next" $\operatorname{Cab}(15,2)$.

$$
\overrightarrow{\mathrm{r}}=\{3,2\}, \quad \overrightarrow{\mathrm{s}}=\{2,3\}, \quad \mathcal{T}=\operatorname{Cab}(15,2) T(3,2) ;
$$

$$
\begin{array}{r}
\mathcal{H}_{\vec{r}, \vec{s}}(\square ; q, t, a)=1+q t+q^{2} t+q^{3} t+q^{2} t^{2}+q^{3} t^{2}+2 q^{4} t^{2}+q^{3} t^{3}+q^{4} t^{3} \\
+2 q^{5} t^{3}+q^{4} t^{4}+q^{5} t^{4}+2 q^{6} t^{4}+q^{5} t^{5}+q^{6} t^{5}+2 q^{7} t^{5} \\
+q^{6} t^{6}+q^{7} t^{6}+q^{8} t^{6}+q^{7} t^{7}+q^{8} t^{7}+q^{8} t^{8}+q^{9} t^{9} \\
+a^{3}\left(q^{6}+q^{7} t+q^{8} t^{2}+q^{9} t^{3}\right) \\
+a^{2}\left(q^{3}+q^{4}+q^{5}+q^{4} t+2 q^{5} t+2 q^{6} t+q^{5} t^{2}+2 q^{6} t^{2}+2 q^{7} t^{2}+q^{6} t^{3}\right. \\
\left.+2 q^{7} t^{3}+2 q^{8} t^{3}+q^{7} t^{4}+2 q^{8} t^{4}+q^{9} t^{4}+q^{8} t^{5}+q^{9} t^{5}+q^{9} t^{6}\right)
\end{array}
$$

\section{Betti numbers}

$$
\mathcal{H}(q=1, t, a=0)=1+3 t+4 t^{2}+4 t^{3}+4 t^{4}+4 t^{5}+3 t^{6}+2 t^{7}+t^{8}+t^{9}
$$

\section{Khovanov-Rozansky polynomials}

(2) $D A H A^{\prime} \operatorname{Kh}(\operatorname{Cab}(15,2) T(3,2))=1+q^{4} t^{2}+q^{6} t^{3}+q^{6} t^{4}$

$$
+q^{10} t^{5}+q^{8} t^{6}+q^{12} t^{7}+2 q^{12} t^{8}+2 q^{14} t^{9}+q^{18} t^{11}+q^{20} t^{12},
$$

$D A H A^{\prime} K_{h R}(C a b(15,2) T(3,2))=1+q^{4} t^{2}+q^{8} t^{3}+q^{6} t^{4}+q^{8} t^{4}$

$$
\begin{aligned}
& +q^{10} t^{5}+q^{12} t^{5}+q^{8} t^{6}+q^{10} t^{6}+2 q^{14} t^{7}+q^{16} t^{7}+2 q^{12} t^{8}+q^{14} t^{8}+q^{18} t^{8} \\
& +2 q^{16} t^{9}+2 q^{18} t^{9}+q^{16} t^{10}+q^{22} t^{10}+2 q^{20} t^{11}+q^{22} t^{11}+q^{24} t^{12}+q^{26} t^{12} .
\end{aligned}
$$

\subsection{Degree 5 and beyond}

The $a$-degree was 3 in the previous examples; let us consider two examples of uncolored DAHA superpolynomials of degree 5 (which is $s_{1} r_{2}-1$ in these cases) and then some further examples of DAHA-Betti polynomials, which are beyond known or conjectured formulas. We note that $D A H A^{\prime} K h R$ do not coincide with the reduced (tilde-normalized) Khovanov polynomials $\widetilde{K} h$ in these two examples, but the structure of the difference matches our conjecture.

$$
\vec{r}=\{3,3\}, \quad \vec{s}=\{2,1\}, \quad \mathcal{T}=\operatorname{Cab}(19,3) T(3,2)
$$


$\mathcal{H}_{\vec{r}, \overrightarrow{\mathrm{s}}}(\square ; q, t, a)=1+q t+q^{2} t+q^{3} t+q^{4} t+q^{5} t+q^{2} t^{2}+q^{3} t^{2}+2 q^{4} t^{2}+2 q^{5} t^{2}+3 q^{6} t^{2}$ $+2 q^{7} t^{2}+q^{8} t^{2}+q^{3} t^{3}+q^{4} t^{3}+2 q^{5} t^{3}+3 q^{6} t^{3}+4 q^{7} t^{3}+4 q^{8} t^{3}+4 q^{9} t^{3}+q^{10} t^{3}+q^{4} t^{4}$ $+q^{5} t^{4}+2 q^{6} t^{4}+3 q^{7} t^{4}+5 q^{8} t^{4}+5 q^{9} t^{4}+6 q^{10} t^{4}+4 q^{11} t^{4}+q^{12} t^{4}+q^{5} t^{5}+q^{6} t^{5}$ $+2 q^{7} t^{5}+3 q^{8} t^{5}+5 q^{9} t^{5}+6 q^{10} t^{5}+7 q^{11} t^{5}+6 q^{12} t^{5}+3 q^{13} t^{5}+q^{6} t^{6}+q^{7} t^{6}+2 q^{8} t^{6}$ $+3 q^{9} t^{6}+5 q^{10} t^{6}+6 q^{11} t^{6}+8 q^{12} t^{6}+7 q^{13} t^{6}+3 q^{14} t^{6}+q^{15} t^{6}+q^{7} t^{7}+q^{8} t^{7}+2 q^{9} t^{7}$ $+3 q^{10} t^{7}+5 q^{11} t^{7}+6 q^{12} t^{7}+8 q^{13} t^{7}+8 q^{14} t^{7}+3 q^{15} t^{7}+q^{8} t^{8}+q^{9} t^{8}+2 q^{10} t^{8}$ $+3 q^{11} t^{8}+5 q^{12} t^{8}+6 q^{13} t^{8}+8 q^{14} t^{8}+7 q^{15} t^{8}+3 q^{16} t^{8}+q^{9} t^{9}+q^{10} t^{9}+2 q^{11} t^{9}$ $+3 q^{12} t^{9}+5 q^{13} t^{9}+6 q^{14} t^{9}+8 q^{15} t^{9}+6 q^{16} t^{9}+q^{17} t^{9}+q^{10} t^{10}+q^{11} t^{10}+2 q^{12} t^{10}$ $+3 q^{13} t^{10}+5 q^{14} t^{10}+6 q^{15} t^{10}+7 q^{16} t^{10}+4 q^{17} t^{10}+q^{11} t^{11}+q^{12} t^{11}+2 q^{13} t^{11}$ $+3 q^{14} t^{11}+5 q^{15} t^{11}+6 q^{16} t^{11}+6 q^{17} t^{11}+q^{18} t^{11}+q^{12} t^{12}+q^{13} t^{12}+2 q^{14} t^{12}$ $+3 q^{15} t^{12}+5 q^{16} t^{12}+5 q^{17} t^{12}+4 q^{18} t^{12}+q^{13} t^{13}+q^{14} t^{13}+2 q^{15} t^{13}+3 q^{16} t^{13}$ $+5 q^{17} t^{13}+4 q^{18} t^{13}+q^{19} t^{13}+q^{14} t^{14}+q^{15} t^{14}+2 q^{16} t^{14}+3 q^{17} t^{14}+4 q^{18} t^{14}$ $+2 q^{19} t^{14}+q^{15} t^{15}+q^{16} t^{15}+2 q^{17} t^{15}+3 q^{18} t^{15}+3 q^{19} t^{15}+q^{16} t^{16}+q^{17} t^{16}$ $+2 q^{18} t^{16}+2 q^{19} t^{16}+q^{20} t^{16}+q^{17} t^{17}+q^{18} t^{17}+2 q^{19} t^{17}+q^{20} t^{17}+q^{18} t^{18}$ $+q^{19} t^{18}+q^{20} t^{18}+q^{19} t^{19}+q^{20} t^{19}+q^{20} t^{20}+q^{21} t^{21}$ $+a^{5}\left(q^{15}+q^{16} t+q^{17} t+q^{17} t^{2}+q^{18} t^{2}+q^{19} t^{2}+q^{18} t^{3}+q^{19} t^{3}+q^{19} t^{4}+q^{20} t^{4}+q^{20} t^{5}+q^{21} t^{6}\right)$ $+a^{4}\left(q^{10}+q^{11}+q^{12}+q^{13}+q^{14}+q^{11} t+2 q^{12} t+3 q^{13} t+3 q^{14} t+3 q^{15} t+q^{16} t+q^{12} t^{2}\right.$

$$
\begin{aligned}
& +2 q^{13} t^{2}+4 q^{14} t^{2}+5 q^{15} t^{2}+5 q^{16} t^{2}+3 q^{17} t^{2}+q^{18} t^{2}+q^{13} t^{3}+2 q^{14} t^{3}+4 q^{15} t^{3} \\
& +6 q^{16} t^{3}+6 q^{17} t^{3}+3 q^{18} t^{3}+q^{19} t^{3}+q^{14} t^{4}+2 q^{15} t^{4}+4 q^{16} t^{4}+6 q^{17} t^{4}+6 q^{18} t^{4}
\end{aligned}
$$$$
+3 q^{19} t^{4}+q^{15} t^{5}+2 q^{16} t^{5}+4 q^{17} t^{5}+6 q^{18} t^{5}+5 q^{19} t^{5}+q^{20} t^{5}+q^{16} t^{6}+2 q^{17} t^{6}
$$$$
+4 q^{18} t^{6}+5 q^{19} t^{6}+3 q^{20} t^{6}+q^{17} t^{7}+2 q^{18} t^{7}+4 q^{19} t^{7}+3 q^{20} t^{7}+q^{21} t^{7}+q^{18} t^{8}
$$$$
\left.+2 q^{19} t^{8}+3 q^{20} t^{8}+q^{21} t^{8}+q^{19} t^{9}+2 q^{20} t^{9}+q^{21} t^{9}+q^{20} t^{10}+q^{21} t^{10}+q^{21} t^{11}\right)
$$

$+a^{3}\left(q^{6}+q^{7}+2 q^{8}+2 q^{9}+2 q^{10}+q^{11}+q^{12}+q^{7} t+2 q^{8} t+4 q^{9} t+6 q^{10} t+7 q^{11} t\right.$

$$
\begin{array}{r}
+4 q^{13} t+2 q^{14} t+q^{8} t^{2}+2 q^{9} t^{2}+5 q^{10} t^{2}+8 q^{11} t^{2}+12 q^{12} t^{2}+12 q^{13} t^{2}+10 q^{14} t^{2} \\
+5 q^{15} t^{2}+2 q^{16} t^{2}+q^{9} t^{3}+2 q^{10} t^{3}+5 q^{11} t^{3}+9 q^{12} t^{3}+14 q^{13} t^{3}+17 q^{14} t^{3}
\end{array}
$$

$+15 q^{15} t^{3}+9 q^{16} t^{3}+3 q^{17} t^{3}+q^{18} t^{3}+q^{10} t^{4}+2 q^{11} t^{4}+5 q^{12} t^{4}+9 q^{13} t^{4}+15 q^{14} t^{4}$

$+19 q^{15} t^{4}+18 q^{16} t^{4}+10 q^{17} t^{4}+3 q^{18} t^{4}+q^{11} t^{5}+2 q^{12} t^{5}+5 q^{13} t^{5}+9 q^{14} t^{5}$

$+15 q^{15} t^{5}+20 q^{16} t^{5}+18 q^{17} t^{5}+9 q^{18} t^{5}+2 q^{19} t^{5}+q^{12} t^{6}+2 q^{13} t^{6}+5 q^{14} t^{6}$ $+9 q^{15} t^{6}+15 q^{16} t^{6}+19 q^{17} t^{6}+15 q^{18} t^{6}+5 q^{19} t^{6}+q^{13} t^{7}+2 q^{14} t^{7}+5 q^{15} t^{7}$ $+9 q^{16} t^{7}+15 q^{17} t^{7}+17 q^{18} t^{7}+10 q^{19} t^{7}+2 q^{20} t^{7}+q^{14} t^{8}+2 q^{15} t^{8}+5 q^{16} t^{8}$ $+9 q^{17} t^{8}+14 q^{18} t^{8}+12 q^{19} t^{8}+4 q^{20} t^{8}+q^{15} t^{9}+2 q^{16} t^{9}+5 q^{17} t^{9}+9 q^{18} t^{9}$ $+12 q^{19} t^{9}+6 q^{20} t^{9}+q^{21} t^{9}+q^{16} t^{10}+2 q^{17} t^{10}+5 q^{18} t^{10}+8 q^{19} t^{10}+7 q^{20} t^{10}$ $+q^{21} t^{10}+q^{17} t^{11}+2 q^{18} t^{11}+5 q^{19} t^{11}+6 q^{20} t^{11}+2 q^{21} t^{11}+q^{18} t^{12}+2 q^{19} t^{12}$ $\left.+4 q^{20} t^{12}+2 q^{21} t^{12}+q^{19} t^{13}+2 q^{20} t^{13}+2 q^{21} t^{13}+q^{20} t^{14}+q^{21} t^{14}+q^{21} t^{15}\right)$ 
$+a^{2}\left(q^{3}+q^{4}+2 q^{5}+2 q^{6}+2 q^{7}+q^{8}+q^{9}+q^{4} t+2 q^{5} t+4 q^{6} t+6 q^{7} t+8 q^{8} t+7 q^{9} t+6 q^{10} t\right.$

$+3 q^{11} t+q^{12} t+q^{5} t^{2}+2 q^{6} t^{2}+5 q^{7} t^{2}+8 q^{8} t^{2}+13 q^{9} t^{2}+15 q^{10} t^{2}+15 q^{11} t^{2}+10 q^{12} t^{2}$ $+5 q^{13} t^{2}+q^{14} t^{2}+q^{6} t^{3}+2 q^{7} t^{3}+5 q^{8} t^{3}+9 q^{9} t^{3}+15 q^{10} t^{3}+20 q^{11} t^{3}+24 q^{12} t^{3}+19 q^{13} t^{3}$ $+11 q^{14} t^{3}+4 q^{15} t^{3}+q^{16} t^{3}+q^{7} t^{4}+2 q^{8} t^{4}+5 q^{9} t^{4}+9 q^{10} t^{4}+16 q^{11} t^{4}+22 q^{12} t^{4}+29 q^{13} t^{4}$ $+27 q^{14} t^{4}+16 q^{15} t^{4}+6 q^{16} t^{4}+q^{17} t^{4}+q^{8} t^{5}+2 q^{9} t^{5}+5 q^{10} t^{5}+9 q^{11} t^{5}+16 q^{12} t^{5}+23 q^{13} t^{5}$ $+31 q^{14} t^{5}+30 q^{15} t^{5}+19 q^{16} t^{5}+6 q^{17} t^{5}+q^{18} t^{5}+q^{9} t^{6}+2 q^{10} t^{6}+5 q^{11} t^{6}+9 q^{12} t^{6}+16 q^{13} t^{6}$ $+23 q^{14} t^{6}+32 q^{15} t^{6}+30 q^{16} t^{6}+16 q^{17} t^{6}+4 q^{18} t^{6}+q^{10} t^{7}+2 q^{11} t^{7}+5 q^{12} t^{7}+9 q^{13} t^{7}$ $+16 q^{14} t^{7}+23 q^{15} t^{7}+31 q^{16} t^{7}+27 q^{17} t^{7}+11 q^{18} t^{7}+q^{19} t^{7}+q^{11} t^{8}+2 q^{12} t^{8}+5 q^{13} t^{8}$ $+9 q^{14} t^{8}+16 q^{15} t^{8}+23 q^{16} t^{8}+29 q^{17} t^{8}+19 q^{18} t^{8}+5 q^{19} t^{8}+q^{12} t^{9}+2 q^{13} t^{9}+5 q^{14} t^{9}$ $+9 q^{15} t^{9}+16 q^{16} t^{9}+22 q^{17} t^{9}+24 q^{18} t^{9}+10 q^{19} t^{9}+q^{20} t^{9}+q^{13} t^{10}+2 q^{14} t^{10}+5 q^{15} t^{10}$ $+9 q^{16} t^{10}+16 q^{17} t^{10}+20 q^{18} t^{10}+15 q^{19} t^{10}+3 q^{20} t^{10}+q^{14} t^{11}+2 q^{15} t^{11}+5 q^{16} t^{11}$ $+9 q^{17} t^{11}+15 q^{18} t^{11}+15 q^{19} t^{11}+6 q^{20} t^{11}+q^{15} t^{12}+2 q^{16} t^{12}+5 q^{17} t^{12}+9 q^{18} t^{12}+13 q^{19} t^{12}$ $+7 q^{20} t^{12}+q^{21} t^{12}+q^{16} t^{13}+2 q^{17} t^{13}+5 q^{18} t^{13}+8 q^{19} t^{13}+8 q^{20} t^{13}+q^{21} t^{13}+q^{17} t^{14}$ $+2 q^{18} t^{14}+5 q^{19} t^{14}+6 q^{20} t^{14}+2 q^{21} t^{14}+q^{18} t^{15}+2 q^{19} t^{15}+4 q^{20} t^{15}+2 q^{21} t^{15}+q^{19} t^{16}$ $\left.+2 q^{20} t^{16}+2 q^{21} t^{16}+q^{20} t^{17}+q^{21} t^{17}+q^{21} t^{18}\right)$ $+a\left(q+q^{2}+q^{3}+q^{4}+q^{5}+q^{2} t+2 q^{3} t+3 q^{4} t+4 q^{5} t+5 q^{6} t+4 q^{7} t+2 q^{8} t+q^{9} t+q^{3} t^{2}+2 q^{4} t^{2}\right.$ $+4 q^{5} t^{2}+6 q^{6} t^{2}+9 q^{7} t^{2}+10 q^{8} t^{2}+9 q^{9} t^{2}+5 q^{10} t^{2}+2 q^{11} t^{2}+q^{4} t^{3}+2 q^{5} t^{3}+4 q^{6} t^{3}+7 q^{7} t^{3}$ $+11 q^{8} t^{3}+14 q^{9} t^{3}+16 q^{10} t^{3}+13 q^{11} t^{3}+6 q^{12} t^{3}+2 q^{13} t^{3}+q^{5} t^{4}+2 q^{6} t^{4}+4 q^{7} t^{4}+7 q^{8} t^{4}$ $+12 q^{9} t^{4}+16 q^{10} t^{4}+20 q^{11} t^{4}+20 q^{12} t^{4}+13 q^{13} t^{4}+4 q^{14} t^{4}+q^{15} t^{4}+q^{6} t^{5}+2 q^{7} t^{5}+4 q^{8} t^{5}$ $+7 q^{9} t^{5}+12 q^{10} t^{5}+17 q^{11} t^{5}+22 q^{12} t^{5}+24 q^{13} t^{5}+17 q^{14} t^{5}+7 q^{15} t^{5}+q^{16} t^{5}+q^{7} t^{6}+2 q^{8} t^{6}$ $+4 q^{9} t^{6}+7 q^{10} t^{6}+12 q^{11} t^{6}+17 q^{12} t^{6}+23 q^{13} t^{6}+26 q^{14} t^{6}+18 q^{15} t^{6}+7 q^{16} t^{6}+q^{17} t^{6}+q^{8} t^{7}$ $+2 q^{9} t^{7}+4 q^{10} t^{7}+7 q^{11} t^{7}+12 q^{12} t^{7}+17 q^{13} t^{7}+23 q^{14} t^{7}+26 q^{15} t^{7}+17 q^{16} t^{7}+4 q^{17} t^{7}$ $+q^{9} t^{8}+2 q^{10} t^{8}+4 q^{11} t^{8}+7 q^{12} t^{8}+12 q^{13} t^{8}+17 q^{14} t^{8}+23 q^{15} t^{8}+24 q^{16} t^{8}+13 q^{17} t^{8}$ $+2 q^{18} t^{8}+q^{10} t^{9}+2 q^{11} t^{9}+4 q^{12} t^{9}+7 q^{13} t^{9}+12 q^{14} t^{9}+17 q^{15} t^{9}+22 q^{16} t^{9}+20 q^{17} t^{9}$ $+6 q^{18} t^{9}+q^{11} t^{10}+2 q^{12} t^{10}+4 q^{13} t^{10}+7 q^{14} t^{10}+12 q^{15} t^{10}+17 q^{16} t^{10}+20 q^{17} t^{10}+13 q^{18} t^{10}$ $+2 q^{19} t^{10}+q^{12} t^{11}+2 q^{13} t^{11}+4 q^{14} t^{11}+7 q^{15} t^{11}+12 q^{16} t^{11}+16 q^{17} t^{11}+16 q^{18} t^{11}+5 q^{19} t^{11}$ $+q^{13} t^{12}+2 q^{14} t^{12}+4 q^{15} t^{12}+7 q^{16} t^{12}+12 q^{17} t^{12}+14 q^{18} t^{12}+9 q^{19} t^{12}+q^{20} t^{12}+q^{14} t^{13}$ $+2 q^{15} t^{13}+4 q^{16} t^{13}+7 q^{17} t^{13}+11 q^{18} t^{13}+10 q^{19} t^{13}+2 q^{20} t^{13}+q^{15} t^{14}+2 q^{16} t^{14}+4 q^{17} t^{14}$ $+7 q^{18} t^{14}+9 q^{19} t^{14}+4 q^{20} t^{14}+q^{16} t^{15}+2 q^{17} t^{15}+4 q^{18} t^{15}+6 q^{19} t^{15}+5 q^{20} t^{15}+q^{17} t^{16}$ $+2 q^{18} t^{16}+4 q^{19} t^{16}+4 q^{20} t^{16}+q^{21} t^{16}+q^{18} t^{17}+2 q^{19} t^{17}+3 q^{20} t^{17}+q^{21} t^{17}+q^{19} t^{18}$ $\left.+2 q^{20} t^{18}+q^{21} t^{18}+q^{20} t^{19}+q^{21} t^{19}+q^{21} t^{20}\right)$.

We note that examples involving torus knots and cables different from $T(2 m+1,2)$, $\operatorname{Cab}(2 m+1,2)(\cdot)$ are important, since the knots/cables of types $(*, 2)$ and their invariants are generally very special. 


\section{Betti numbers (Conjecturally)}

$$
\begin{aligned}
\mathcal{H}(q=1, t, a=0)=1+5 t & +12 t^{2}+20 t^{3}+28 t^{4}+34 t^{5}+37 t^{6} \\
+37 t^{7}+36 t^{8} & +33 t^{9}+29 t^{10}+25 t^{11}+21 t^{12}+17 t^{13}+13 t^{14} \\
+ & 10 t^{15}+7 t^{16}+5 t^{17}+3 t^{18}+2 t^{19}+t^{20}+t^{21}
\end{aligned}
$$

The germ $\mathcal{C}_{\vec{r}, \vec{s}}$ is given here by the local ring $\mathcal{R}=\mathbb{C} \llbracket z^{6}, z^{9}+z^{10} \rrbracket$ with the Puiseux exponents $(6,9,10)$, the valuation semigroup $\Gamma$ generated by $6,9,19$ and $\delta=|\mathbb{N} \backslash \Gamma|=$ 21 , which matches the top $t$-degree in the DAHA-Betti polynomial from (3-3). Thus, the Euler number of $J\left(\mathcal{C}_{\vec{r}, \vec{s}}\right)$ is 365 (the value in (3-3) at $t=1$ ), which follows from [34; 30], and we predict that the Betti numbers of $J\left(\mathcal{C}_{\vec{r}, \vec{s}}\right)$ are the coefficients of this polynomial. This example is from the table after [36, Theorem 13] with some cases where the approach there is not applicable (including counting the Euler number).

\section{Khovanov polynomial}

$$
\begin{aligned}
& D A H A^{\prime} K h(\operatorname{Cab}(19,3) T(3,2))=1+q^{4} t^{2}+q^{6} t^{3}+q^{6} t^{4}+q^{10} t^{5}+q^{8} t^{6} \\
&+ q^{12} t^{7}+q^{10} t^{8}+q^{12} t^{8}+2 q^{14} t^{9}+q^{12} t^{10}+q^{14} t^{10}+2 q^{16} t^{11}+q^{18} t^{11} \\
&+ 3 q^{16} t^{12}+q^{20} t^{12}+2 q^{18} t^{13}+2 q^{20} t^{13}+2 q^{18} t^{14}+q^{22} t^{14}+4 q^{22} t^{15} \\
&+ q^{20} t^{16}+2 q^{22} t^{16}+q^{24} t^{16}+q^{26} t^{16}+4 q^{24} t^{17}+3 q^{24} t^{18}+q^{28} t^{18} \\
&+ 3 q^{26} t^{19}+2 q^{28} t^{19}+q^{26} t^{20}+q^{28} t^{20}+2 q^{30} t^{20}+4 q^{30} t^{21}+2 q^{30} t^{22} \\
&+2 q^{32} t^{22}+3 q^{32} t^{23}+q^{32} t^{24}+q^{36} t^{24}+2 q^{36} t^{25}+q^{34} t^{26} \\
&+q^{36} t^{26}+2 q^{38} t^{26}+q^{36} t^{27}+q^{38} t^{27}+q^{40} t^{27}+q^{40} t^{29}+q^{42} t^{30}
\end{aligned}
$$

This one is now different from the actual Khovanov polynomial:

$$
\begin{aligned}
\widetilde{K} h-D A H A^{\prime} K h=q^{34} t^{23}\left(1+2 t-t^{3}\right)+q^{36} t^{22} & \left(1-t^{2}\right) \\
& +q^{40} t^{27}\left(1-t^{2}\right)+q^{42} t^{28}\left(1-t^{2}\right) .
\end{aligned}
$$

There is no coincidence, but the $t$-degree corrections involve only top terms and all are in the expected direction. As it was discussed after the Connection conjecture, if the difference $\widetilde{K h}-D A H A^{\prime} K h$ is a sum of the terms $q^{i} t^{j}(1+t)$ and $q^{i} t^{j}\left(1-t^{2}\right)$ with positive coefficients, then this is a confirmation of part (ii). Here and below (where Khovanov polynomials are discussed), $q$ and $t$ are actually $q_{s t}$ and $t_{s t}$. This is what can be expected, taking into consideration the nature of the procedure from [12] (and the example of $T(12,7)$ there).

$\mathcal{H}_{\{4,2\},\{3,1\}}$ The next example will be of the same $\operatorname{deg}_{a}=5$. Recall that $\operatorname{deg}_{a} \leq$ $\left(s_{1} r_{2}-1\right)$ in the uncolored case with 2 iterations; conjecturally the coincidence holds here for algebraic knots. Generally, $\left(s_{1} r_{2}-1\right)$ must be multiplied by ord $\left(\lambda_{b}\right)$. 


$$
\overrightarrow{\mathrm{r}}=\{4,2\}, \quad \overrightarrow{\mathrm{s}}=\{3,1\}, \quad \mathcal{T}=\operatorname{Cab}(25,2) T(4,3)
$$

$\mathcal{H}_{\overrightarrow{\mathrm{r}}, \overrightarrow{\mathrm{s}}}(\square ; q, t, a)=1+q t+q^{2} t+q^{3} t+q^{4} t+q^{5} t+q^{2} t^{2}+q^{3} t^{2}+2 q^{4} t^{2}+2 q^{5} t^{2}+3 q^{6} t^{2}+q^{7} t^{2}$ $+q^{8} t^{2}+q^{3} t^{3}+q^{4} t^{3}+2 q^{5} t^{3}+3 q^{6} t^{3}+4 q^{7} t^{3}+3 q^{8} t^{3}+3 q^{9} t^{3}+q^{4} t^{4}+q^{5} t^{4}+2 q^{6} t^{4}+3 q^{7} t^{4}$ $+5 q^{8} t^{4}+4 q^{9} t^{4}+5 q^{10} t^{4}+q^{11} t^{4}+q^{5} t^{5}+q^{6} t^{5}+2 q^{7} t^{5}+3 q^{8} t^{5}+5 q^{9} t^{5}+5 q^{10} t^{5}+6 q^{11} t^{5}$ $+q^{12} t^{5}+q^{6} t^{6}+q^{7} t^{6}+2 q^{8} t^{6}+3 q^{9} t^{6}+5 q^{10} t^{6}+5 q^{11} t^{6}+7 q^{12} t^{6}+q^{13} t^{6}+q^{7} t^{7}+q^{8} t^{7}$ $+2 q^{9} t^{7}+3 q^{10} t^{7}+5 q^{11} t^{7}+5 q^{12} t^{7}+6 q^{13} t^{7}+q^{14} t^{7}+q^{8} t^{8}+q^{9} t^{8}+2 q^{10} t^{8}+3 q^{11} t^{8}$ $+5 q^{12} t^{8}+5 q^{13} t^{8}+5 q^{14} t^{8}+q^{9} t^{9}+q^{10} t^{9}+2 q^{11} t^{9}+3 q^{12} t^{9}+5 q^{13} t^{9}+4 q^{14} t^{9}+3 q^{15} t^{9}$ $+q^{10} t^{10}+q^{11} t^{10}+2 q^{12} t^{10}+3 q^{13} t^{10}+5 q^{14} t^{10}+3 q^{15} t^{10}+q^{16} t^{10}+q^{11} t^{11}+q^{12} t^{11}$ $+2 q^{13} t^{11}+3 q^{14} t^{11}+4 q^{15} t^{11}+q^{16} t^{11}+q^{12} t^{12}+q^{13} t^{12}+2 q^{14} t^{12}+3 q^{15} t^{12}+3 q^{16} t^{12}$ $+q^{13} t^{13}+q^{14} t^{13}+2 q^{15} t^{13}+2 q^{16} t^{13}+q^{17} t^{13}+q^{14} t^{14}+q^{15} t^{14}+2 q^{16} t^{14}+q^{17} t^{14}$ $+q^{15} t^{15}+q^{16} t^{15}+q^{17} t^{15}+q^{16} t^{16}+q^{17} t^{16}+q^{17} t^{17}+q^{18} t^{18}$

$+a^{5}\left(q^{15}+q^{16} t+q^{17} t^{2}+q^{18} t^{3}\right)$

$+a^{4}\left(q^{10}+q^{11}+q^{12}+q^{13}+q^{14}+q^{11} t+2 q^{12} t+2 q^{13} t+2 q^{14} t+2 q^{15} t+q^{12} t^{2}+2 q^{13} t^{2}\right.$

$$
+3 q^{14} t^{2}+3 q^{15} t^{2}+2 q^{16} t^{2}+q^{13} t^{3}+2 q^{14} t^{3}+3 q^{15} t^{3}+3 q^{16} t^{3}+2 q^{17} t^{3}+q^{14} t^{4}
$$

$$
\begin{aligned}
+2 q^{15} t^{4}+3 q^{16} t^{4}+2 q^{17} t^{4}+q^{18} t^{4} & +q^{15} t^{5}+2 q^{16} t^{5}+2 q^{17} t^{5}+q^{18} t^{5}+q^{16} t^{6} \\
& \left.+2 q^{17} t^{6}+q^{18} t^{6}+q^{17} t^{7}+q^{18} t^{7}+q^{18} t^{8}\right)
\end{aligned}
$$

$+a^{3}\left(q^{6}+q^{7}+2 q^{8}+2 q^{9}+2 q^{10}+q^{11}+q^{12}+q^{7} t+2 q^{8} t+4 q^{9} t+5 q^{10} t+6 q^{11} t+4 q^{12} t\right.$

$+3 q^{13} t+q^{14} t+q^{8} t^{2}+2 q^{9} t^{2}+5 q^{10} t^{2}+7 q^{11} t^{2}+10 q^{12} t^{2}+7 q^{13} t^{2}+5 q^{14} t^{2}+q^{15} t^{2}+q^{9} t^{3}$ $+2 q^{10} t^{3}+5 q^{11} t^{3}+8 q^{12} t^{3}+12 q^{13} t^{3}+9 q^{14} t^{3}+6 q^{15} t^{3}+q^{16} t^{3}+q^{10} t^{4}+2 q^{11} t^{4}+5 q^{12} t^{4}$ $+8 q^{13} t^{4}+13 q^{14} t^{4}+9 q^{15} t^{4}+5 q^{16} t^{4}+q^{17} t^{4}+q^{11} t^{5}+2 q^{12} t^{5}+5 q^{13} t^{5}+8 q^{14} t^{5}+12 q^{15} t^{5}$ $+7 q^{16} t^{5}+3 q^{17} t^{5}+q^{12} t^{6}+2 q^{13} t^{6}+5 q^{14} t^{6}+8 q^{15} t^{6}+10 q^{16} t^{6}+4 q^{17} t^{6}+q^{18} t^{6}+q^{13} t^{7}$

$+2 q^{14} t^{7}+5 q^{15} t^{7}+7 q^{16} t^{7}+6 q^{17} t^{7}+q^{18} t^{7}+q^{14} t^{8}+2 q^{15} t^{8}+5 q^{16} t^{8}+5 q^{17} t^{8}+2 q^{18} t^{8}$ $\left.+q^{15} t^{9}+2 q^{16} t^{9}+4 q^{17} t^{9}+2 q^{18} t^{9}+q^{16} t^{10}+2 q^{17} t^{10}+2 q^{18} t^{10}+q^{17} t^{11}+q^{18} t^{11}+q^{18} t^{12}\right)$ $+a^{2}\left(q^{3}+q^{4}+2 q^{5}+2 q^{6}+2 q^{7}+q^{8}+q^{9}+q^{4} t+2 q^{5} t+4 q^{6} t+6 q^{7} t+7 q^{8} t+6 q^{9} t+5 q^{10} t\right.$

$+2 q^{11} t+q^{12} t+q^{5} t^{2}+2 q^{6} t^{2}+5 q^{7} t^{2}+8 q^{8} t^{2}+12 q^{9} t^{2}+12 q^{10} t^{2}+11 q^{11} t^{2}+5 q^{12} t^{2}$ $+2 q^{13} t^{2}+q^{6} t^{3}+2 q^{7} t^{3}+5 q^{8} t^{3}+9 q^{9} t^{3}+14 q^{10} t^{3}+17 q^{11} t^{3}+17 q^{12} t^{3}+8 q^{13} t^{3}+3 q^{14} t^{3}$ $+q^{7} t^{4}+2 q^{8} t^{4}+5 q^{9} t^{4}+9 q^{10} t^{4}+15 q^{11} t^{4}+19 q^{12} t^{4}+20 q^{13} t^{4}+9 q^{14} t^{4}+3 q^{15} t^{4}+q^{8} t^{5}$ $+2 q^{9} t^{5}+5 q^{10} t^{5}+9 q^{11} t^{5}+15 q^{12} t^{5}+20 q^{13} t^{5}+20 q^{14} t^{5}+8 q^{15} t^{5}+2 q^{16} t^{5}+q^{9} t^{6}+2 q^{10} t^{6}$ $+5 q^{11} t^{6}+9 q^{12} t^{6}+15 q^{13} t^{6}+19 q^{14} t^{6}+17 q^{15} t^{6}+5 q^{16} t^{6}+q^{17} t^{6}+q^{10} t^{7}+2 q^{11} t^{7}+5 q^{12} t^{7}$ $+9 q^{13} t^{7}+15 q^{14} t^{7}+17 q^{15} t^{7}+11 q^{16} t^{7}+2 q^{17} t^{7}+q^{11} t^{8}+2 q^{12} t^{8}+5 q^{13} t^{8}+9 q^{14} t^{8}$ $+14 q^{15} t^{8}+12 q^{16} t^{8}+5 q^{17} t^{8}+q^{12} t^{9}+2 q^{13} t^{9}+5 q^{14} t^{9}+9 q^{15} t^{9}+12 q^{16} t^{9}+6 q^{17} t^{9}+q^{18} t^{9}$ $+q^{13} t^{10}+2 q^{14} t^{10}+5 q^{15} t^{10}+8 q^{16} t^{10}+7 q^{17} t^{10}+q^{18} t^{10}+q^{14} t^{11}+2 q^{15} t^{11}+5 q^{16} t^{11}$ $+6 q^{17} t^{11}+2 q^{18} t^{11}+q^{15} t^{12}+2 q^{16} t^{12}+4 q^{17} t^{12}+2 q^{18} t^{12}+q^{16} t^{13}+2 q^{17} t^{13}+2 q^{18} t^{13}$ $\left.+q^{17} t^{14}+q^{18} t^{14}+q^{18} t^{15}\right)$ 


$$
\begin{array}{r}
+a\left(q+q^{2}+q^{3}+q^{4}+q^{5}+q^{2} t+2 q^{3} t+3 q^{4} t+4 q^{5} t+5 q^{6} t+3 q^{7} t+2 q^{8} t+q^{9} t+q^{3} t^{2}+2 q^{4} t^{2}\right. \\
+4 q^{5} t^{2}+6 q^{6} t^{2}+9 q^{7} t^{2}+8 q^{8} t^{2}+7 q^{9} t^{2}+3 q^{10} t^{2}+q^{11} t^{2}+q^{4} t^{3}+2 q^{5} t^{3}+4 q^{6} t^{3}+7 q^{7} t^{3} \\
+11 q^{8} t^{3}+12 q^{9} t^{3}+13 q^{10} t^{3}+7 q^{11} t^{3}+2 q^{12} t^{3}+q^{5} t^{4}+2 q^{6} t^{4}+4 q^{7} t^{4}+7 q^{8} t^{4}+12 q^{9} t^{4} \\
+14 q^{10} t^{4}+17 q^{11} t^{4}+10 q^{12} t^{4}+3 q^{13} t^{4}+q^{6} t^{5}+2 q^{7} t^{5}+4 q^{8} t^{5}+7 q^{9} t^{5}+12 q^{10} t^{5}+15 q^{11} t^{5} \\
+19 q^{12} t^{5}+11 q^{13} t^{5}+3 q^{14} t^{5}+q^{7} t^{6}+2 q^{8} t^{6}+4 q^{9} t^{6}+7 q^{10} t^{6}+12 q^{11} t^{6}+15 q^{12} t^{6}+19 q^{13} t^{6} \\
+10 q^{14} t^{6}+2 q^{15} t^{6}+q^{8} t^{7}+2 q^{9} t^{7}+4 q^{10} t^{7}+7 q^{11} t^{7}+12 q^{12} t^{7}+15 q^{13} t^{7}+17 q^{14} t^{7}+7 q^{15} t^{7} \\
+q^{16} t^{7}+q^{9} t^{8}+2 q^{10} t^{8}+4 q^{11} t^{8}+7 q^{12} t^{8}+12 q^{13} t^{8}+14 q^{14} t^{8}+13 q^{15} t^{8}+3 q^{16} t^{8}+q^{10} t^{9} \\
+2 q^{11} t^{9}+4 q^{12} t^{9}+7 q^{13} t^{9}+12 q^{14} t^{9}+12 q^{15} t^{9}+7 q^{16} t^{9}+q^{17} t^{9}+q^{11} t^{10}+2 q^{12} t^{10} \\
+4 q^{13} t^{10}+7 q^{14} t^{10}+11 q^{15} t^{10}+8 q^{16} t^{10}+2 q^{17} t^{10}+q^{12} t^{11}+2 q^{13} t^{11}+4 q^{14} t^{11}+7 q^{15} t^{11} \\
+9 q^{16} t^{11}+3 q^{17} t^{11}+q^{13} t^{12}+2 q^{14} t^{12}+4 q^{15} t^{12}+6 q^{16} t^{12}+5 q^{17} t^{12}+q^{14} t^{13}+2 q^{15} t^{13} \\
+4 q^{16} t^{13}+4 q^{17} t^{13}+q^{18} t^{13}+q^{15} t^{14}+2 q^{16} t^{14}+3 q^{17} t^{14}+q^{18} t^{14}+q^{16} t^{15} \\
\left.+2 q^{17} t^{15}+q^{18} t^{15}+q^{17} t^{16}+q^{18} t^{16}+q^{18} t^{17}\right) .
\end{array}
$$

\section{Betti numbers (Conjecturally)}

$$
\begin{aligned}
\mathcal{H}(q=1, t, a=0)=1+5 t & +11 t^{2}+17 t^{3}+22 t^{4}+24 t^{5}+25 t^{6} \\
& +24 t^{7}+22 t^{8}+19 t^{9}+16 t^{10}+12 t^{11}+10 t^{12} \\
& +7 t^{13}+5 t^{14}+3 t^{15}+2 t^{16}+t^{17}+t^{18}
\end{aligned}
$$

The corresponding (germ of the) curve is given by the ring $\mathcal{R}=\mathbb{C} \llbracket z^{6}, z^{8}+z^{9} \rrbracket$ in this case, with the Puiseux exponents $(6,8,9)$ and the valuation semigroup $\Gamma$ generated by $6,8,25$. Accordingly, $\delta=|\mathbb{N} \backslash \Gamma|=18$ in the considered case, which matches the top $t$-degree in (3-4).

Furthermore, $\mathcal{H}(q=1, t=1, a=0)=227$, which does coincide with the specialization at $v=9$ of the general formula for the Euler number $e\left(J\left(\mathcal{C}_{\vec{r}, \vec{s}}\right)\right)=229 / 2+25 v / 2$ in the case of the Puiseux exponents $(6,8, v)$. This formula is from [36, Main theorem]; its proof was technically involved and was not extended to the Betti numbers. We conjecture that they are the $t$-coefficients in (3-4) in this case $(v=9)$.

\section{Khovanov polynomial}

(4) $D A H A^{\prime} \operatorname{Kh}(\operatorname{Cab}(25,2) T(4,3))=1+q^{4} t^{2}+q^{6} t^{3}+q^{6} t^{4}+q^{10} t^{5}+q^{8} t^{6}$

$$
\begin{array}{r}
+q^{12} t^{7}+q^{10} t^{8}+q^{12} t^{8}+2 q^{14} t^{9}+q^{12} t^{10}+q^{14} t^{10}+2 q^{16} t^{11}+q^{18} t^{11} \\
+3 q^{16} t^{12}+q^{20} t^{12}+2 q^{18} t^{13}+2 q^{20} t^{13}+q^{18} t^{14}+q^{20} t^{14}+q^{22} t^{14} \\
+4 q^{22} t^{15}+q^{20} t^{16}+q^{22} t^{16}+q^{24} t^{16}+q^{26} t^{16}+2 q^{24} t^{17}+q^{26} t^{17} \\
+2 q^{24} t^{18}+q^{28} t^{18}+2 q^{26} t^{19}+q^{28} t^{19}+2 q^{28} t^{20}+q^{30} t^{20}+3 q^{30} t^{21} \\
+q^{32} t^{22}+q^{34} t^{23}+q^{32} t^{24}+q^{36} t^{24}+q^{34} t^{25}+q^{36} t^{26}+q^{38} t^{27}
\end{array}
$$


This one is different from the corresponding Khovanov polynomial as well as in the previous example. Namely,

$\widetilde{K h}-D A H A^{\prime} K h=q^{32} t^{22}\left(1-t^{2}\right)+q^{34} t^{23}\left(1-t^{2}\right)+q^{36} t^{24}\left(1-t^{2}\right)+q^{38} t^{25}\left(1-t^{2}\right)$.

Once again, the $t$-degrees increase from $\widetilde{K h}$ to $D A H A^{\prime} K h$; also, the number of $q, t-$ monomials is the same in both, but this seems accidental. In spite of the nonzero difference, this confirms Conjecture 2.4.

Further Betti numbers First of all, our DAHA-Betti polynomials (as far as we calculated them) fully match the table before [36, Conjecture 23]. For instance, the coefficients of $\mathcal{H}_{\{5,2\},\{2,1\}}(q=1, t, a=0)$ for $\operatorname{Cab}(21,2) T(5,2)$ constitute the line there for the Puiseux exponents $(4,10,11)$. Without posting the (known) formulas for the superpolynomials, let us provide below only the DAHA-Betti polynomials, which conjecturally give the corresponding Betti numbers.

$\mathcal{H}_{\{4,2\},\{3,1\}}$ In this example, the knot, the ring of the singularity, the valuation semigroup and the arithmetic genus are as follows:

$$
\mathcal{T}=\operatorname{Cab}(20,3) T(3,2), \quad \mathcal{R}=C \llbracket t^{6}, t^{9}+t^{11} \rrbracket, \quad \Gamma=\langle 6,9,20\rangle, \quad \delta=22 .
$$

The counterpart of the DAHA-Betti polynomial (3-3) reads

$$
\begin{aligned}
\mathcal{H}(q=1, t, a=0) & =1+5 t+12 t^{2}+20 t^{3}+28 t^{4}+35 t^{5}+39 t^{6}+40 t^{7} \\
& +39 t^{8}+37 t^{9}+33 t^{10}+29 t^{11}+25 t^{12}+21 t^{13}+17 t^{14} \\
& +13 t^{15}+10 t^{16}+7 t^{17}+5 t^{18}+3 t^{19}+2 t^{20}+t^{21}+t^{22}
\end{aligned}
$$

The arithmetic genus of $\mathcal{C}=\mathcal{C}_{\{3,3\},\{2,2\}}$ equals $\delta=22$ and $e(J(\mathcal{C}))=423$ in this case; the Puiseux exponents are $(6,9,11)$, and $\operatorname{deg}_{a}=5$.

$\mathcal{H}_{\{\mathbf{5 , 2}\},\{\mathbf{3}, \mathbf{2}\}}$ The Puiseux exponents are $(6,15,17)$, and $\operatorname{deg}_{a}=5$. One has

$$
\mathcal{T}=\operatorname{Cab}(32,3) T(5,2), \quad \mathcal{R}=\mathbb{C} \llbracket z^{6}, z^{15}+z^{17} \rrbracket, \quad \Gamma=\langle 6,15,32\rangle, \quad \delta=37,
$$

and the DAHA-Betti polynomial is

$$
\begin{aligned}
\mathcal{H}(q=1, & t, a=0)=1+5 t+15 t^{2}+32 t^{3}+55 t^{4}+81 t^{5}+108 t^{6} \\
& +134 t^{7}+157 t^{8}+175 t^{9}+186 t^{10}+192 t^{11}+192 t^{12}+189 t^{13} \\
+ & 181 t^{14}+172 t^{15}+159 t^{16}+147 t^{17}+132 t^{18}+120 t^{19}+105 t^{20} \\
+ & 93 t^{21}+79 t^{22}+68 t^{23}+56 t^{24}+47 t^{25}+37 t^{26}+30 t^{27}+23 t^{28} \\
+ & 18 t^{29}+13 t^{30}+10 t^{31}+7 t^{32}+5 t^{33}+3 t^{34}+2 t^{35}+t^{36}+t^{37}
\end{aligned}
$$

The Euler number of $J\left(\mathcal{C}_{\{5,2\},\{3,2\}}\right)$ is 3031 . 
$\mathcal{H}_{\{\mathbf{3}, \mathbf{4}\},\{\mathbf{2}, \mathbf{1}\}}$ In this case, $\operatorname{deg}_{a}=7$ and the Puiseux exponents are $(8,12,13)$. One has

$$
\mathcal{T}=\operatorname{Cab}(25,4) T(3,2), \quad \mathcal{R}=\mathbb{C} \llbracket z^{8}, z^{12}+z^{13} \rrbracket, \quad \Gamma=\langle 8,12,25\rangle, \quad \delta=40,
$$

and the DAHA-Betti polynomial is

$$
\begin{aligned}
\mathcal{H}(q=1, t, a=0) & =1+7 t+24 t^{2}+56 t^{3}+104 t^{4}+165 t^{5}+232 t^{6} \\
+297 & t^{7}+355 t^{8}+402 t^{9}+435 t^{10}+454 t^{11}+461 t^{12}+456 t^{13} \\
& +442 t^{14}+420 t^{15}+394 t^{16}+362 t^{17}+330 t^{18}+295 t^{19} \\
& +262 t^{20}+229 t^{21}+199 t^{22}+168 t^{23}+143 t^{24}+118 t^{25} \\
+ & 97 t^{26}+78 t^{27}+63 t^{28}+48 t^{29}+38 t^{30}+28 t^{31}+21 t^{32} \\
& +15 t^{33}+11 t^{34}+7 t^{35}+5 t^{36}+3 t^{37}+2 t^{38}+t^{39}+t^{40}
\end{aligned}
$$

The Euler number of $J\left(\mathcal{C}_{\{3,4\},\{2,1\}}\right)$ is 7229 . The latter follows from [34; 30]; the Betti numbers are conjectured.

Double iteration: $\overrightarrow{\mathbf{r}}=\{\mathbf{3}, \mathbf{2}, \mathbf{2}\}, \overrightarrow{\mathbf{s}}=\{\mathbf{2}, \mathbf{1}, \mathbf{1}\}$ The corresponding knot is $\mathcal{T}=$ $\operatorname{Cab}(53,2) \operatorname{Cab}(13,2) T(3,2)$ and $\operatorname{deg}_{a}=7$. In this case,

$$
\mathcal{R}=\mathbb{C} \llbracket z^{8}, z^{12}+z^{14}+z^{15} \rrbracket, \quad \Gamma=\langle 8,12,26,53\rangle, \quad \delta=42,
$$

and the DAHA-Betti polynomial is

$$
\begin{aligned}
\mathcal{H}(q=1, t, a=0)=1+7 t+24 t^{2}+56 t^{3}+104 t^{4}+166 t^{5} \\
+236 t^{6}+306 t^{7}+370 t^{8}+424 t^{9}+465 t^{10}+492 t^{11}+507 t^{12} \\
+510 t^{13}+504 t^{14}+488 t^{15}+466 t^{16}+437 t^{17}+406 t^{18}+370 t^{19} \\
+335 t^{20}+298 t^{21}+264 t^{22}+230 t^{23}+199 t^{24}+168 t^{25}+143 t^{26} \\
+118 t^{27}+97 t^{28}+78 t^{29}+63 t^{30}+48 t^{31}+38 t^{32}+28 t^{33} \\
+21 t^{34}+15 t^{35}+11 t^{36}+7 t^{37}+5 t^{38}+3 t^{39}+2 t^{40}+t^{41}+t^{42}
\end{aligned}
$$

The Euler number of $J\left(\mathcal{C}_{\{3,2,2\},\{2,1,1\}}\right)$ is 8512 . This case is listed in [36] (right before Section 4) as an example beyond the technique there.

We omit the complete DAHA superpolynomials and the DAHA-Khovanov polynomials in this and the previous examples (they are all known). Quite a few interesting relations for the (conjectural) Betti numbers can be seen from these and other examples of the DAHA-Betti polynomials. For instance, we note that $b_{2}$ of $J(\mathcal{C})$ coincides with the multiplicity of the corresponding singularity minus 1 (Rego) and therefore exactly with $\operatorname{deg}_{a}=\mathrm{s}_{1} \mathrm{r}_{2} \cdots \mathrm{r}_{\ell}$ of the DAHA-superpolynomial $\left(\mathrm{s}_{1}<\mathrm{r}_{1}\right)$. 


\subsection{The case of two boxes}

We will now consider the iterated knot from the first example with the weight $2 \omega_{1}$. We calculated the one for $\omega_{2}$ too; it is superdual to the one we provide and will be omitted. Also, we will omit the corresponding DAHA-Khovanov polynomial (calculated using the algorithm from [12]), since the Khovanov polynomials are uncolored.

$$
\overrightarrow{\mathrm{r}}=\{3,2\}, \quad \overrightarrow{\mathrm{s}}=\{2,1\}, \quad \mathcal{T}=\operatorname{Cab}(13,2) T(3,2) ;
$$

$\mathcal{H}_{\overrightarrow{\mathrm{r}}, \overrightarrow{\mathrm{s}}}(\square \square ; q, t, a)=1+t^{16} q^{32}+t^{15} q^{31}+t^{14} q^{31}+t^{13} q^{31}+t^{15} q^{30}+2 t^{14} q^{30}+2 t^{13} q^{30}$ $+2 t^{12} q^{30}+t^{14} q^{29}+3 t^{13} q^{29}+4 t^{12} q^{29}+3 t^{11} q^{29}+t^{14} q^{28}+2 t^{13} q^{28}+5 t^{12} q^{28}+6 t^{11} q^{28}$ $+4 t^{10} q^{28}+t^{13} q^{27}+3 t^{12} q^{27}+7 t^{11} q^{27}+8 t^{10} q^{27}+4 t^{9} q^{27}+t^{13} q^{26}+2 t^{12} q^{26}+5 t^{11} q^{26}$ $+10 t^{10} q^{26}+9 t^{9} q^{26}+2 t^{8} q^{26}+t^{12} q^{25}+3 t^{11} q^{25}+7 t^{10} q^{25}+12 t^{9} q^{25}+7 t^{8} q^{25}+t^{7} q^{25}$ $+t^{12} q^{24}+2 t^{11} q^{24}+5 t^{10} q^{24}+10 t^{9} q^{24}+14 t^{8} q^{24}+4 t^{7} q^{24}+t^{11} q^{23}+3 t^{10} q^{23}+7 t^{9} q^{23}$ $+12 t^{8} q^{23}+11 t^{7} q^{23}+t^{6} q^{23}+t^{11} q^{22}+2 t^{10} q^{22}+5 t^{9} q^{22}+10 t^{8} q^{22}+13 t^{7} q^{22}+6 t^{6} q^{22}$ $+t^{10} q^{21}+3 t^{9} q^{21}+7 t^{8} q^{21}+12 t^{7} q^{21}+10 t^{6} q^{21}+t^{5} q^{21}+t^{10} q^{20}+2 t^{9} q^{20}+5 t^{8} q^{20}$ $+10 t^{7} q^{20}+13 t^{6} q^{20}+3 t^{5} q^{20}+t^{9} q^{19}+3 t^{8} q^{19}+7 t^{7} q^{19}+11 t^{6} q^{19}+9 t^{5} q^{19}+t^{9} q^{18}$ $+2 t^{8} q^{18}+5 t^{7} q^{18}+10 t^{6} q^{18}+11 t^{5} q^{18}+2 t^{4} q^{18}+t^{8} q^{17}+3 t^{7} q^{17}+7 t^{6} q^{17}+10 t^{5} q^{17}$ $+4 t^{4} q^{17}+t^{8} q^{16}+2 t^{7} q^{16}+5 t^{6} q^{16}+9 t^{5} q^{16}+8 t^{4} q^{16}+t^{7} q^{15}+3 t^{6} q^{15}+7 t^{5} q^{15}+8 t^{4} q^{15}$ $+2 t^{3} q^{15}+t^{7} q^{14}+2 t^{6} q^{14}+5 t^{5} q^{14}+8 t^{4} q^{14}+3 t^{3} q^{14}+t^{6} q^{13}+3 t^{5} q^{13}+6 t^{4} q^{13}+5 t^{3} q^{13}$ $+t^{6} q^{12}+2 t^{5} q^{12}+5 t^{4} q^{12}+6 t^{3} q^{12}+t^{2} q^{12}+t^{5} q^{11}+3 t^{4} q^{11}+5 t^{3} q^{11}+t^{2} q^{11}+t^{5} q^{10}$ $+2 t^{4} q^{10}+4 t^{3} q^{10}+3 t^{2} q^{10}+t^{4} q^{9}+3 t^{3} q^{9}+3 t^{2} q^{9}+t^{4} q^{8}+2 t^{3} q^{8}+3 t^{2} q^{8}+t^{3} q^{7}+2 t^{2} q^{7}$ $+t q^{7}+t^{3} q^{6}+2 t^{2} q^{6}+t q^{6}+t^{2} q^{5}+t q^{5}+t^{2} q^{4}+t q^{4}+t q^{3}+t q^{2}$ $+a^{6}\left(t^{4} q^{35}+t^{3} q^{34}+t^{3} q^{33}+t^{2} q^{33}+t^{2} q^{32}+t^{2} q^{31}+t q^{30}+t q^{29}+q^{27}\right)$ $+a^{5}\left(t^{7} q^{35}+t^{6} q^{35}+t^{5} q^{35}+t^{7} q^{34}+2 t^{6} q^{34}+2 t^{5} q^{34}+t^{4} q^{34}+2 t^{6} q^{33}+4 t^{5} q^{33}+4 t^{4} q^{33}\right.$ $+t^{3} q^{33}+t^{6} q^{32}+4 t^{5} q^{32}+5 t^{4} q^{32}+3 t^{3} q^{32}+2 t^{5} q^{31}+5 t^{4} q^{31}+5 t^{3} q^{31}+t^{2} q^{31}+t^{5} q^{30}$ $+4 t^{4} q^{30}+6 t^{3} q^{30}+3 t^{2} q^{30}+2 t^{4} q^{29}+6 t^{3} q^{29}+5 t^{2} q^{29}+t^{4} q^{28}+4 t^{3} q^{28}+5 t^{2} q^{28}+t q^{28}$ $+2 t^{3} q^{27}+5 t^{2} q^{27}+3 t q^{27}+t^{3} q^{26}+4 t^{2} q^{26}+3 t q^{26}+2 t^{2} q^{25}+3 t q^{25}+q^{25}+t^{2} q^{24}+3 t q^{24}$ $\left.+q^{24}+2 t q^{23}+q^{23}+t q^{22}+q^{22}+q^{21}+q^{20}\right)$ $+a^{4}\left(t^{9} q^{35}+t^{8} q^{35}+t^{7} q^{35}+t^{10} q^{34}+2 t^{9} q^{34}+4 t^{8} q^{34}+3 t^{7} q^{34}+2 t^{6} q^{34}+2 t^{9} q^{33}+5 t^{8} q^{33}\right.$ $+8 t^{7} q^{33}+6 t^{6} q^{33}+3 t^{5} q^{33}+t^{9} q^{32}+5 t^{8} q^{32}+11 t^{7} q^{32}+13 t^{6} q^{32}+8 t^{5} q^{32}+2 t^{4} q^{32}$ $+2 t^{8} q^{31}+9 t^{7} q^{31}+16 t^{6} q^{31}+15 t^{5} q^{31}+6 t^{4} q^{31}+t^{3} q^{31}+t^{8} q^{30}+5 t^{7} q^{30}+16 t^{6} q^{30}$ $+21 t^{5} q^{30}+14 t^{4} q^{30}+3 t^{3} q^{30}+2 t^{7} q^{29}+9 t^{6} q^{29}+22 t^{5} q^{29}+20 t^{4} q^{29}+8 t^{3} q^{29}+t^{7} q^{28}$ $+5 t^{6} q^{28}+16 t^{5} q^{28}+25 t^{4} q^{28}+14 t^{3} q^{28}+2 t^{2} q^{28}+2 t^{6} q^{27}+9 t^{5} q^{27}+21 t^{4} q^{27}+20 t^{3} q^{27}$ $+5 t^{2} q^{27}+t^{6} q^{26}+5 t^{5} q^{26}+16 t^{4} q^{26}+22 t^{3} q^{26}+10 t^{2} q^{26}+2 t^{5} q^{25}+9 t^{4} q^{25}+20 t^{3} q^{25}$ $+13 t^{2} q^{25}+2 t q^{25}+t^{5} q^{24}+5 t^{4} q^{24}+15 t^{3} q^{24}+17 t^{2} q^{24}+4 t q^{24}+2 t^{4} q^{23}+9 t^{3} q^{23}$ 


$$
\begin{array}{r}
+15 t^{2} q^{23}+6 t q^{23}+t^{4} q^{22}+5 t^{3} q^{22}+13 t^{2} q^{22}+8 t q^{22}+q^{22}+2 t^{3} q^{21}+8 t^{2} q^{21}+9 t q^{21} \\
+q^{21}+t^{3} q^{20}+5 t^{2} q^{20}+8 t q^{20}+2 q^{20}+2 t^{2} q^{19}+6 t q^{19}+2 q^{19}+t^{2} q^{18}+4 t q^{18}+3 q^{18} \\
\left.+5 t^{2} q^{20}+8 t q^{20}+2 q^{20}+2 t^{2} q^{19}+2 t q^{17}+2 q^{17}+t q^{16}+2 q^{16}+q^{15}+q^{14}\right)
\end{array}
$$

$+a^{3}\left(t^{10} q^{35}+t^{12} q^{34}+2 t^{11} q^{34}+3 t^{10} q^{34}+3 t^{9} q^{34}+t^{8} q^{34}+t^{12} q^{33}+3 t^{11} q^{33}+6 t^{10} q^{33}\right.$

$+8 t^{9} q^{33}+6 t^{8} q^{33}+2 t^{7} q^{33}+2 t^{11} q^{32}+8 t^{10} q^{32}+14 t^{9} q^{32}+16 t^{8} q^{32}+10 t^{7} q^{32}+3 t^{6} q^{32}$ $+t^{11} q^{31}+5 t^{10} q^{31}+15 t^{9} q^{31}+25 t^{8} q^{31}+22 t^{7} q^{31}+11 t^{6} q^{31}+2 t^{5} q^{31}+2 t^{10} q^{30}+10 t^{9} q^{30}$

$+26 t^{8} q^{30}+36 t^{7} q^{30}+26 t^{6} q^{30}+9 t^{5} q^{30}+t^{4} q^{30}+t^{10} q^{29}+5 t^{9} q^{29}+18 t^{8} q^{29}+39 t^{7} q^{29}$ $+42 t^{6} q^{29}+21 t^{5} q^{29}+4 t^{4} q^{29}+2 t^{9} q^{28}+10 t^{8} q^{28}+29 t^{7} q^{28}+50 t^{6} q^{28}+39 t^{5} q^{28}+12 t^{4} q^{28}$ $+t^{3} q^{28}+t^{9} q^{27}+5 t^{8} q^{27}+18 t^{7} q^{27}+43 t^{6} q^{27}+52 t^{5} q^{27}+25 t^{4} q^{27}+4 t^{3} q^{27}+2 t^{8} q^{26}$ $+10 t^{7} q^{26}+29 t^{6} q^{26}+53 t^{5} q^{26}+41 t^{4} q^{26}+10 t^{3} q^{26}+t^{8} q^{25}+5 t^{7} q^{25}+18 t^{6} q^{25}+43 t^{5} q^{25}$ $+50 t^{4} q^{25}+20 t^{3} q^{25}+t^{2} q^{25}+2 t^{7} q^{24}+10 t^{6} q^{24}+29 t^{5} q^{24}+50 t^{4} q^{24}+32 t^{3} q^{24}+5 t^{2} q^{24}$ $+t^{7} q^{23}+5 t^{6} q^{23}+18 t^{5} q^{23}+41 t^{4} q^{23}+39 t^{3} q^{23}+10 t^{2} q^{23}+2 t^{6} q^{22}+10 t^{5} q^{22}+28 t^{4} q^{22}$ $+41 t^{3} q^{22}+18 t^{2} q^{22}+t q^{22}+t^{6} q^{21}+5 t^{5} q^{21}+18 t^{4} q^{21}+36 t^{3} q^{21}+24 t^{2} q^{21}+3 t q^{21}$ $+2 t^{5} q^{20}+10 t^{4} q^{20}+26 t^{3} q^{20}+27 t^{2} q^{20}+6 t q^{20}+t^{5} q^{19}+5 t^{4} q^{19}+17 t^{3} q^{19}+26 t^{2} q^{19}$ $+9 t q^{19}+2 t^{4} q^{18}+10 t^{3} q^{18}+21 t^{2} q^{18}+12 t q^{18}+q^{18}+t^{4} q^{17}+5 t^{3} q^{17}+15 t^{2} q^{17}+13 t q^{17}$ $+q^{17}+2 t^{3} q^{16}+9 t^{2} q^{16}+12 t q^{16}+2 q^{16}+t^{3} q^{15}+5 t^{2} q^{15}+10 t q^{15}+3 q^{15}+2 t^{2} q^{14}+7 t q^{14}$ $\left.+3 q^{14}+t^{2} q^{13}+4 t q^{13}+3 q^{13}+2 t q^{12}+3 q^{12}+t q^{11}+2 q^{11}+q^{10}+q^{9}\right)$ $+a^{2}\left(t^{13} q^{34}+t^{12} q^{34}+t^{11} q^{34}+t^{14} q^{33}+2 t^{13} q^{33}+4 t^{12} q^{33}+4 t^{11} q^{33}+3 t^{10} q^{33}+2 t^{13} q^{32}\right.$ $+5 t^{12} q^{32}+10 t^{11} q^{32}+10 t^{10} q^{32}+6 t^{9} q^{32}+t^{8} q^{32}+t^{13} q^{31}+5 t^{12} q^{31}+12 t^{11} q^{31}+20 t^{10} q^{31}$ $+18 t^{9} q^{31}+8 t^{8} q^{31}+t^{7} q^{31}+2 t^{12} q^{30}+9 t^{11} q^{30}+21 t^{10} q^{30}+32 t^{9} q^{30}+24 t^{8} q^{30}+9 t^{7} q^{30}$ $+t^{6} q^{30}+t^{12} q^{29}+5 t^{11} q^{29}+17 t^{10} q^{29}+35 t^{9} q^{29}+45 t^{8} q^{29}+25 t^{7} q^{29}+7 t^{6} q^{29}+2 t^{11} q^{28}$ $+9 t^{10} q^{28}+27 t^{9} q^{28}+49 t^{8} q^{28}+49 t^{7} q^{28}+20 t^{6} q^{28}+3 t^{5} q^{28}+t^{11} q^{27}+5 t^{10} q^{27}+17 t^{9} q^{27}$ $+42 t^{8} q^{27}+62 t^{7} q^{27}+44 t^{6} q^{27}+11 t^{5} q^{27}+t^{4} q^{27}+2 t^{10} q^{26}+9 t^{9} q^{26}+27 t^{8} q^{26}+57 t^{7} q^{26}$ $+63 t^{6} q^{26}+28 t^{5} q^{26}+3 t^{4} q^{26}+t^{10} q^{25}+5 t^{9} q^{25}+17 t^{8} q^{25}+42 t^{7} q^{25}+70 t^{6} q^{25}+48 t^{5} q^{25}$ $+11 t^{4} q^{25}+2 t^{9} q^{24}+9 t^{8} q^{24}+27 t^{7} q^{24}+56 t^{6} q^{24}+66 t^{5} q^{24}+24 t^{4} q^{24}+2 t^{3} q^{24}+t^{9} q^{23}$ $+5 t^{8} q^{23}+17 t^{7} q^{23}+42 t^{6} q^{23}+67 t^{5} q^{23}+42 t^{4} q^{23}+6 t^{3} q^{23}+2 t^{8} q^{22}+9 t^{7} q^{22}+27 t^{6} q^{22}$ $+55 t^{5} q^{22}+54 t^{4} q^{22}+15 t^{3} q^{22}+t^{8} q^{21}+5 t^{7} q^{21}+17 t^{6} q^{21}+41 t^{5} q^{21}+60 t^{4} q^{21}+26 t^{3} q^{21}$

$+2 t^{2} q^{21}+2 t^{7} q^{20}+9 t^{6} q^{20}+27 t^{5} q^{20}+50 t^{4} q^{20}+38 t^{3} q^{20}+5 t^{2} q^{20}+t^{7} q^{19}+5 t^{6} q^{19}$ $+17 t^{5} q^{19}+39 t^{4} q^{19}+43 t^{3} q^{19}+12 t^{2} q^{19}+2 t^{6} q^{18}+9 t^{5} q^{18}+26 t^{4} q^{18}+41 t^{3} q^{18}+18 t^{2} q^{18}$ $+t q^{18}+t^{6} q^{17}+5 t^{5} q^{17}+17 t^{4} q^{17}+34 t^{3} q^{17}+25 t^{2} q^{17}+2 t q^{17}+2 t^{5} q^{16}+9 t^{4} q^{16}+24 t^{3} q^{16}$ $+25 t^{2} q^{16}+5 t q^{16}+t^{5} q^{15}+5 t^{4} q^{15}+16 t^{3} q^{15}+25 t^{2} q^{15}+8 t q^{15}+2 t^{4} q^{14}+9 t^{3} q^{14}+19 t^{2} q^{14}$ $+10 t q^{14}+t^{4} q^{13}+5 t^{3} q^{13}+14 t^{2} q^{13}+11 t q^{13}+q^{13}+2 t^{3} q^{12}+8 t^{2} q^{12}+11 t q^{12}+q^{12}+t^{3} q^{11}$ $+5 t^{2} q^{11}+9 t q^{11}+2 q^{11}+2 t^{2} q^{10}+6 t q^{10}+2 q^{10}+t^{2} q^{9}+4 t q^{9}+3 q^{9}+2 t q^{8}+2 q^{8}+t q^{7}$ $\left.+2 q^{7}+q^{6}+q^{5}\right)$ 


$$
\begin{aligned}
& +a\left(t^{15} q^{33}+t^{14} q^{33}+t^{13} q^{33}+t^{15} q^{32}+2 t^{14} q^{32}+3 t^{13} q^{32}+3 t^{12} q^{32}+t^{11} q^{32}+2 t^{14} q^{31}\right. \\
& +5 t^{13} q^{31}+8 t^{12} q^{31}+7 t^{11} q^{31}+2 t^{10} q^{31}+t^{14} q^{30}+4 t^{13} q^{30}+9 t^{12} q^{30}+14 t^{11} q^{30} \\
& +11 t^{10} q^{30}+3 t^{9} q^{30}+2 t^{13} q^{29}+7 t^{12} q^{29}+16 t^{11} q^{29}+22 t^{10} q^{29}+15 t^{9} q^{29}+3 t^{8} q^{29} \\
& +t^{13} q^{28}+4 t^{12} q^{28}+12 t^{11} q^{28}+24 t^{10} q^{28}+29 t^{9} q^{28}+14 t^{8} q^{28}+2 t^{7} q^{28}+2 t^{12} q^{27} \\
& +7 t^{11} q^{27}+19 t^{10} q^{27}+34 t^{9} q^{27}+32 t^{8} q^{27}+11 t^{7} q^{27}+t^{6} q^{27}+t^{12} q^{26}+4 t^{11} q^{26} \\
& +12 t^{10} q^{26}+28 t^{9} q^{26}+42 t^{8} q^{26}+27 t^{7} q^{26}+5 t^{6} q^{26}+2 t^{11} q^{25}+7 t^{10} q^{25}+19 t^{9} q^{25} \\
& +38 t^{8} q^{25}+43 t^{7} q^{25}+17 t^{6} q^{25}+t^{5} q^{25}+t^{11} q^{24}+4 t^{10} q^{24}+12 t^{9} q^{24}+28 t^{8} q^{24}+46 t^{7} q^{24} \\
& +34 t^{6} q^{24}+6 t^{5} q^{24}+2 t^{10} q^{23}+7 t^{9} q^{23}+19 t^{8} q^{23}+38 t^{7} q^{23}+46 t^{6} q^{23}+17 t^{5} q^{23}+t^{4} q^{23} \\
& +t^{10} q^{22}+4 t^{9} q^{22}+12 t^{8} q^{22}+28 t^{7} q^{22}+45 t^{6} q^{22}+31 t^{5} q^{22}+4 t^{4} q^{22}+2 t^{9} q^{21}+7 t^{8} q^{21} \\
& +19 t^{7} q^{21}+37 t^{6} q^{21}+42 t^{5} q^{21}+11 t^{4} q^{21}+t^{9} q^{20}+4 t^{8} q^{20}+12 t^{7} q^{20}+28 t^{6} q^{20}+42 t^{5} q^{20} \\
& +21 t^{4} q^{20}+t^{3} q^{20}+2 t^{8} q^{19}+7 t^{7} q^{19}+19 t^{6} q^{19}+35 t^{5} q^{19}+32 t^{4} q^{19}+5 t^{3} q^{19}+t^{8} q^{18} \\
& +4 t^{7} q^{18}+12 t^{6} q^{18}+27 t^{5} q^{18}+35 t^{4} q^{18}+11 t^{3} q^{18}+2 t^{7} q^{17}+7 t^{6} q^{17}+19 t^{5} q^{17}+31 t^{4} q^{17} \\
& +18 t^{3} q^{17}+t^{2} q^{17}+t^{7} q^{16}+4 t^{6} q^{16}+12 t^{5} q^{16}+25 t^{4} q^{16}+23 t^{3} q^{16}+3 t^{2} q^{16}+2 t^{6} q^{15} \\
& +7 t^{5} q^{15}+18 t^{4} q^{15}+24 t^{3} q^{15}+7 t^{2} q^{15}+t^{6} q^{14}+4 t^{5} q^{14}+12 t^{4} q^{14}+21 t^{3} q^{14}+10 t^{2} q^{14} \\
& +2 t^{5} q^{13}+7 t^{4} q^{13}+16 t^{3} q^{13}+13 t^{2} q^{13}+t q^{13}+t^{5} q^{12}+4 t^{4} q^{12}+11 t^{3} q^{12}+14 t^{2} q^{12} \\
& +2 t q^{12}+2 t^{4} q^{11}+7 t^{3} q^{11}+12 t^{2} q^{11}+3 t q^{11}+t^{4} q^{10}+4 t^{3} q^{10}+9 t^{2} q^{10}+5 t q^{10}+2 t^{3} q^{9} \\
& +6 t^{2} q^{9}+6 t q^{9}+t^{3} q^{8}+4 t^{2} q^{8}+5 t q^{8}+2 t^{2} q^{7}+4 t q^{7}+q^{7}+t^{2} q^{6}+3 t q^{6}+q^{6}+2 t q^{5} \\
& \left.+q^{5}+t q^{4}+q^{4}+q^{3}+q^{2}\right) \text {. }
\end{aligned}
$$

\section{Betti numbers (Presumably)}

$$
\begin{aligned}
\mathcal{H}(q=1, t, a=0)=1+6 t+17 t^{2}+ & 32 t^{3}+48 t^{4}+62 t^{5}+70 t^{6}+70 t^{7} \\
& +64 t^{8}+54 t^{9}+41 t^{10}+28 t^{11}+18 t^{12} \\
& +10 t^{13}+5 t^{14}+2 t^{15}+t^{16} \\
= & \left(1+3 t+4 t^{2}+4 t^{3}+4 t^{4}+3 t^{5}+2 t^{6}+t^{7}+t^{8}\right)^{2} .
\end{aligned}
$$

We put here (and below) "presumably", since at the moment it is not clear which Betti numbers this polynomial calculates (even conjecturally). The last relation is a particular case of part (iv) of Theorem 2.3 (the specialization at $q=1$ ). The full specialization formula in this case reads as follows:

$$
\begin{aligned}
& \mathcal{H}_{\{3,2\},\{2,1\}}\left(2 \omega_{1} ; q=1, t, a\right)=\left(1+3 a+3 a^{2}+a^{3}+3 t+7 a t+5 a^{2} t\right. \\
& +a^{3} t+4 t^{2}+8 a t^{2}+5 a^{2} t^{2}+a^{3} t^{2}+4 t^{3}+8 a t^{3} \\
& +4 a^{2} t^{3}+4 t^{4}+6 a t^{4}+2 a^{2} t^{4}+3 t^{5}+4 a t^{5} \\
& \left.+a^{2} t^{5}+2 t^{6}+2 a t^{6}+t^{7}+a t^{7}+t^{8}\right)^{2} \\
& =\mathcal{H}_{\{3,2\},\{2,1\}}\left(\omega_{1} ; q=1, t, a\right)^{2} \text {. }
\end{aligned}
$$


$\mathcal{H}_{\{\mathbf{3}, \mathbf{2}\},\{\mathbf{2}, \mathbf{1}\}}\left(\boldsymbol{\omega}_{\mathbf{2}}\right)$ The superpolynomial for the same iterated knot and the weight $\omega_{2}$ is connected with the one for $2 \omega_{1}$ by the superduality, so we will not list it. However, let us provide its value at $a=0, q=1$ (presumably a sequence of Betti numbers):

$$
\begin{aligned}
\mathcal{H}_{\{3,2\},\{2,1\}}\left(\omega_{2} ; q=1, t, a=0\right) \\
=1+3 t+7 t^{2}+11 t^{3}+18 t^{4}+23 t^{5}+29 t^{6}+31 t^{7}+36 t^{8}+35 t^{9}+37 t^{10} \\
+34 t^{11}+34 t^{12}+31 t^{13}+31 t^{14}+25 t^{15}+25 t^{16}+21 t^{17} \\
+19 t^{18}+15 t^{19}+15 t^{20}+10 t^{21}+10 t^{22}+7 t^{23}+6 t^{24} \\
+4 t^{25}+4 t^{26}+2 t^{27}+2 t^{28}+t^{29}+t^{30}+t^{32}
\end{aligned}
$$

A geometric meaning of formulas (3-9) and (3-10) in terms of certain Betti numbers remains to be found. Generally, we expect that a variant of the construction from [30; 14] can be used here.

\subsection{Pseudoalgebraic knots}

This section is a theoretical challenge. It indicates that some nonalgebraic iterated knots are similar to the algebraic ones, especially the pseudoalgebraic, defined above as those with positive uncolored DAHA superpolynomials. This class conjecturally includes all algebraic knots and their mirror images.

The two knots below possess such a positivity and all other DAHA properties of algebraic knots; for instance, the existence of the tilde-normalization from Theorem 2.1 for any $A_{N}$ holds, as does the strict equality for $\operatorname{deg}_{a}$ in (2-20). We give below some other examples of pseudoalgebraic knots that do not have these two features; see (4-1) and (4-7).

In spite of the presence of negative $s_{i}$ there, we think that the coefficients of the DAHA-Betti polynomials in (3-11) and (3-12) can be expected to be Betti numbers of Jacobian factors for certain (germs of) curves. Hopefully partial normalizations of $\mathcal{C}_{\vec{r}, \vec{s}}$ will emerge here, however this is unknown at the moment.

$$
\overrightarrow{\mathrm{r}}=\{3,2\}, \quad \overrightarrow{\mathrm{s}}=\{2,-3\}, \quad \mathcal{T}=\operatorname{Cab}(9,2) T(3,2) ;
$$

$$
\begin{array}{r}
\mathcal{H}_{\vec{r}, \vec{s}}(\square ; q, t, a)=1+a^{3} q^{6}+q t+q^{2} t+q^{3} t+q^{2} t^{2}+q^{3} t^{2}+2 q^{4} t^{2}+q^{3} t^{3}+q^{4} t^{3}+q^{5} t^{3} \\
+q^{4} t^{4}+q^{5} t^{4}+q^{5} t^{5}+q^{6} t^{6} \\
+a^{2}\left(q^{3}+q^{4}+q^{5}+q^{4} t+2 q^{5} t+q^{6} t+q^{5} t^{2}+q^{6} t^{2}+q^{6} t^{3}\right) \\
+a\left(q+q^{2}+q^{3}+q^{2} t+2 q^{3} t+3 q^{4} t+q^{5} t+q^{3} t^{2}+2 q^{4} t^{2}\right. \\
\left.+3 q^{5} t^{2}+q^{4} t^{3}+2 q^{5} t^{3}+q^{6} t^{3}+q^{5} t^{4}+q^{6} t^{4}+q^{6} t^{5}\right) .
\end{array}
$$




\section{Betti numbers (Presumably)}

$$
\mathcal{H}(q=1, t, a=0)=1+3 t+4 t^{2}+3 t^{3}+2 t^{4}+t^{5}+t^{6} .
$$

This sequence of coefficients and the one in the next example do not come from algebraic knots. However they fit the table of Betti numbers from [36] for the Puiseux exponents $(4,6, v)$. The first two entries there (our examples (1) and (2)) are for $(4,6,7)$ and $(4,6,9)$, respectively; the knots from (6) and (7) formally correspond to nonalgebraic $(4,6,3)$ and $(4,6,5)$, respectively.

\section{Khovanov polynomial}

(6) $D A H A^{\prime} \operatorname{Kh}(\operatorname{Cab}(9,2) T(3,2))=1+q^{4} t^{2}+q^{6} t^{3}+q^{6} t^{4}+q^{10} t^{5}+q^{8} t^{6}+q^{12} t^{7}$

$$
+2 q^{12} t^{8}+2 q^{14} t^{9}+q^{18} t^{11}+q^{20} t^{12} \text {. }
$$

This polynomial, as well as the next $D A H A^{\prime} K h$, coincide with the corresponding (reduced) Khovanov polynomials. This confirms the Connection conjecture(ii) and also demonstrates that the differentials that are necessary to extract the Khovanov polynomials from the stable ones satisfy assumptions (2.13) from [12, Section 3.6].

$\mathcal{H}_{\{\mathbf{3}, \mathbf{2}\},\{\mathbf{2}, \mathbf{- 1}\}}$ This knot is pseudoalgebraic but nonalgebraic as well. In a sense, it is the greatest nonalgebraic, since $\operatorname{Cab}(13,2) T(3,2)$ from (1) above is the first algebraic one in this series.

$$
\overrightarrow{\mathrm{r}}=\{3,2\}, \quad \overrightarrow{\mathrm{s}}=\{2,-1\}, \quad \mathcal{T}=\operatorname{Cab}(11,2) T(3,2) ;
$$

$$
\begin{array}{r}
\mathcal{H}_{\overrightarrow{\mathrm{r}}, \overrightarrow{\mathrm{s}}}(\square ; q, t, a)=1+q t+q^{2} t+q^{3} t+q^{2} t^{2}+q^{3} t^{2}+2 q^{4} t^{2}+q^{3} t^{3}+q^{4} t^{3}+2 q^{5} t^{3}+q^{4} t^{4} \\
+q^{5} t^{4}+q^{6} t^{4}+q^{5} t^{5}+q^{6} t^{5}+q^{6} t^{6}+q^{7} t^{7}+a^{3}\left(q^{6}+q^{7} t\right) \\
+a^{2}\left(q^{3}+q^{4}+q^{5}+q^{4} t+2 q^{5} t+2 q^{6} t+q^{5} t^{2}+2 q^{6} t^{2}+q^{7} t^{2}+q^{6} t^{3}+q^{7} t^{3}+q^{7} t^{4}\right) \\
+a\left(q+q^{2}+q^{3}+q^{2} t+2 q^{3} t+3 q^{4} t+q^{5} t+q^{3} t^{2}+2 q^{4} t^{2}+4 q^{5} t^{2}+q^{6} t^{2}+q^{4} t^{3}\right. \\
\left.+2 q^{5} t^{3}+3 q^{6} t^{3}+q^{5} t^{4}+2 q^{6} t^{4}+q^{7} t^{4}+q^{6} t^{5}+q^{7} t^{5}+q^{7} t^{6}\right) .
\end{array}
$$

\section{Betti numbers (Presumably)}

$$
\mathcal{H}(q=1, t, a=0)=1+3 t+4 t^{2}+4 t^{3}+3 t^{4}+2 t^{5}+t^{6}+t^{7} .
$$

The general formula is not very difficult to calculate because $q=1$ is the trivial central charge (when there are significant simplifications):

$$
\begin{aligned}
\mathcal{H}_{\{3,2\},\{2,2 n-15\}}(q=1, t, a=0)=1+3 t+ & 4\left(t^{2}+\cdots+t^{n-4}\right) \\
& +3 t^{n-3}+2 t^{n-2}+t^{n-1}+t^{n}
\end{aligned}
$$

for $\operatorname{Cab}(2 n-3,2) T(3,2), n \geq 6$. 


\section{Khovanov polynomial}

(7) $D A H A^{\prime} K h(C a b(11,2) T(3,2))=1+q^{4} t^{2}+q^{6} t^{3}+q^{6} t^{4}+q^{10} t^{5}+q^{8} t^{6}+q^{12} t^{7}$

$$
+2 q^{12} t^{8}+2 q^{14} t^{9}+q^{16} t^{10}+2 q^{18} t^{11}+q^{20} t^{12} \text {. }
$$

This polynomial and the previous one coincide with the corresponding Khovanov polynomials (under the tilde-normalization); recall that we use the standard parameters in all formulas involving Khovanov polynomials. Thus, $q, t$ above are actually $q_{s t}, t_{s t}$.

\section{Further aspects}

\subsection{Nonalgebraic knots}

Continuing the previous section, let us discuss nonalgebraic knots that are not pseudoalgebraic. Though the first knot we will consider is pseudoalgebraic, it has some features that are not expected in algebraic knots. For instance, the corresponding $\operatorname{deg}_{a}$ is strictly smaller than $\left(\left|s_{1} r_{2}\right|-1\right)=3$. The coincidence holds for the similar pseudoalgebraic knots from (6) and (7) above and is conjectured to be true for all algebraic knots.

$$
\overrightarrow{\mathrm{r}}=\{3,-2\}, \quad \overrightarrow{\mathrm{s}}=\{2,5\}, \quad \mathcal{T}=\operatorname{Cab}(7,2) T(3,2) ;
$$

$\mathcal{H}_{\overrightarrow{\mathrm{r}}, \overrightarrow{\mathrm{s}}}(\square ; q, t, a)=1+q t+q^{2} t+q^{3} t+q^{2} t^{2}+q^{3} t^{2}+q^{4} t^{2}+q^{3} t^{3}+q^{4} t^{3}+q^{4} t^{4}+q^{5} t^{5}$

$$
\begin{aligned}
+a^{2}\left(q^{3}+q^{4}+q^{4} t+q^{5} t+q^{5} t^{2}\right) & \\
+a\left(q+q^{2}+q^{3}+q^{2} t+2 q^{3} t+2 q^{4} t\right. & +q^{3} t^{2}+2 q^{4} t^{2}+q^{5} t^{2} \\
& \left.+q^{4} t^{3}+q^{5} t^{3}+q^{5} t^{4}\right) .
\end{aligned}
$$

$$
\begin{aligned}
\mathcal{H}(q=1, t, a=0)= & 1+3 t+3 t^{2}+2 t^{3}+t^{4}+t^{5} \\
\operatorname{DAHA}^{\prime} \operatorname{Kh}(\operatorname{Cab}(7,2) T(3,2))= & 1+q^{4} t^{2}+q^{6} t^{3}+q^{6} t^{4}+q^{10} t^{5}+q^{8} t^{6} \\
& +q^{12} t^{7}+q^{12} t^{8}+q^{14} t^{9}+q^{18} t^{11}+q^{20} t^{12} .
\end{aligned}
$$

We see that the first formula is quite similar to (3-13). The DAHA-Khovanov polynomial coincides here with the actual Khovanov one (reduced and under the tilde normalization); recall that we use the standard parameters in the formulas for the DAHA-Khovanov polynomials.

$$
\overrightarrow{\mathrm{r}}=\{3,-2\}, \quad \overrightarrow{\mathrm{s}}=\{2,7\}, \quad \mathcal{T}=\operatorname{Cab}(5,2) T(3,2) ;
$$

$$
\begin{aligned}
\mathcal{H}_{\overrightarrow{\mathrm{r}}, \overrightarrow{\mathrm{s}}}(\square ; q, t, a)=1-\frac{1}{t} a^{3} q^{5} & +q t+q^{2} t+q^{2} t^{2}+q^{3} t^{2}+q^{3} t^{3}+q^{4} t^{4} \\
& +a^{2}\left(q^{3}-q^{5}-\frac{1}{t} q^{4}+q^{4} t\right) \\
& +a\left(q+q^{2}-q^{4}+q^{2} t+2 q^{3} t+q^{3} t^{2}+q^{4} t^{2}+q^{4} t^{3}\right) .
\end{aligned}
$$




$$
\begin{aligned}
\mathcal{H}(q=1, t, a=0)= & 1+2 t+2 t^{2}+t^{3}+t^{4} \\
\operatorname{DAHA}^{\prime} \operatorname{Kh}(\operatorname{Cab}(5,2) T(3,2))= & 1+q^{4} t^{2}+q^{6} t^{3}+q^{6} t^{4}+q^{10} t^{5}+q^{10} t^{6} \\
& +q^{12} t^{7}+q^{12} t^{8}+q^{14} t^{9}+q^{18} t^{11}+q^{20} t^{12} .
\end{aligned}
$$

The knot $\operatorname{Cab}(5,2) T(3,2)$ is the first with nonpositive $\mathcal{H}_{\vec{r}, \vec{s}}(\square ; q, t, a)$ in the considered family. The actual Khovanov polynomials coincide with $D A H A^{\prime} K h$.

The corresponding DAHA-Jones polynomial for $A_{1}$ is

$$
\begin{aligned}
\widetilde{J D}_{\{3,-2\},\{2,7\}}^{A_{1}}\left(\omega_{1} ; q, t\right) & =\mathcal{H}\left(q, t, a=-t^{2}\right) \\
& =1+q t+q^{2} t-q t^{2}+q^{3} t^{2}+q^{4} t^{2} \\
& \quad-q^{2} t^{3}-q^{3} t^{3}-q^{4} t^{3}-q^{5} t^{4}+q^{5} t^{5} .
\end{aligned}
$$

Its value at $t=q$ is the (reduced) Jones polynomial $\widetilde{J}=1+q^{2}-q^{7}-q^{9}+q^{10}$ (for our $q$ and under the tilde normalization); the (reduced) one produced by the KnotTheory package is $q^{4}+q^{6}-q^{11}-q^{13}+q^{14}$.

We note that (4-3) has little to do with the polynomial obtained in [40, Section 5.2], (for the same knot). We cannot comment on this and the general approach used in [40]. The latter stimulated our paper, but the construction there remains unclear to us.

$$
\overrightarrow{\mathrm{r}}=\{3,-2\}, \quad \overrightarrow{\mathrm{s}}=\{2,9\}, \quad \mathcal{T}=\operatorname{Cab}(3,2) T(3,2) ;
$$

$\mathcal{H}_{\overrightarrow{\mathrm{r}}, \overrightarrow{\mathrm{s}}}(\square ; q, t, a)=1-q^{2}+q t+q^{2} t-q^{3} t+q^{2} t^{2}+q^{3} t^{3}$

$$
\begin{gathered}
+a^{3}\left(-\frac{1}{t^{2}} q^{4}-\frac{1}{t} q^{5}\right)+a^{2}\left(q^{3}-q^{4}-q^{5}-\frac{1}{t^{2}} q^{3}-\frac{1}{t} q^{3}-\frac{1}{t} 2 q^{4}\right) \\
+a\left(q+q^{2}-2 q^{3}-q^{4}-\frac{1}{t} q^{2}-\frac{1}{t} q^{3}+q^{2} t+q^{3} t-q^{4} t+q^{3} t^{2}\right) .
\end{gathered}
$$

$$
\begin{aligned}
\mathcal{H}(q=1, t, a=0) & =t+t^{2}+t^{3} \\
D_{A H A^{\prime}} \operatorname{Kh}(\operatorname{Cab}(3,2) T(3,2)) & =1+q^{4} t^{2}+q^{4} t^{3}+q^{6} t^{3}+2 q^{6} t^{4}+q^{10} t^{5} \\
& +q^{10} t^{6}+q^{12} t^{7}+q^{12} t^{8}+q^{14} t^{9}+q^{18} t^{11}+q^{20} t^{12} .
\end{aligned}
$$

Here, $\widetilde{K h}-D A H A^{\prime} K h=q^{20} t^{12}\left(1-t^{2}\right)$, which matches our expectations.

$$
\overrightarrow{\mathrm{r}}=\{3,-2\}, \quad \overrightarrow{\mathrm{s}}=\{2,11\}, \quad \mathcal{T}=\operatorname{Cab}(1,2) T(3,2) ;
$$

$\mathcal{H}_{\vec{r}, \vec{s}}(\square ; q, t, a)=-q+t-2 q^{2} t+q t^{2}-q^{3} t^{2}+q^{2} t^{3}$

$$
\begin{aligned}
& +a^{3}\left(-q^{5}-\frac{1}{t^{2}} q^{3}-\frac{1}{t} q^{4}\right) \\
& +a^{2}\left(-2 q^{3}-2 q^{4}-\frac{1}{t^{2}} q^{2}-\frac{1}{t} q^{2}-\frac{1}{t} 2 q^{3}-q^{4} t-q^{5} t\right) \\
& +a\left(-3 q^{2}-q^{3}-\frac{1}{t} q-\frac{1}{t} q^{2}+q t-3 q^{3} t-q^{4} t+q^{2} t^{2}-q^{4} t^{2}\right) .
\end{aligned}
$$




$$
\begin{aligned}
\mathcal{H}(q=1, t, a=0)= & -1-t+t^{3}, \\
\widetilde{K} h(\operatorname{Cab}(1,2) T(3,2))= & D A H A^{\prime} \operatorname{Kh}(\operatorname{Cab}(1,2) T(3,2)) \\
= & 1+t+q^{2} t^{2}+q^{4} t^{2}+q^{4} t^{3}+q^{6} t^{3}+2 q^{6} t^{4}+q^{10} t^{5} \\
& \quad+q^{10} t^{6}+q^{12} t^{7}+q^{12} t^{8}+q^{14} t^{9}+q^{18} t^{11}+q^{20} t^{12} .
\end{aligned}
$$$$
\text { (e) } \quad \vec{r}=\{3,-2\}, \quad \vec{s}=\{2,13\}, \quad \mathcal{T}=\operatorname{Cab}(-1,2) T(3,2) \text {; }
$$

$$
\begin{aligned}
& \mathcal{H}_{\overrightarrow{\mathrm{r}}, \overrightarrow{\mathrm{s}}}(\square ; q, t, a)=1+2 q t-t^{2}+q t^{2}+2 q^{2} t^{2}-q t^{3}+q^{3} t^{3} \\
& \quad+a^{3}\left(q^{4}+\frac{1}{t^{2}} q^{2}+\frac{1}{t} q^{3}+q^{5} t\right) \\
& \quad+a^{2}\left(2 q^{2}+2 q^{3}+\frac{1}{t^{2}} q+\frac{1}{t} q+\frac{1}{t} 2 q^{2}+q^{2} t+2 q^{3} t+2 q^{4} t+q^{4} t^{2}+q^{5} t^{2}\right) \\
& \quad+a\left(3 q+q^{2}+\frac{1}{t} 1+\frac{1}{t} q+q t+4 q^{2} t+q^{3} t-q t^{2}+q^{2} t^{2}+3 q^{3} t^{2}+q^{4} t^{2}+q^{4} t^{3}\right) .
\end{aligned}
$$

Note that we have $\mathcal{H}(q=0, t, a=0)=1+a / t$ for $\mathrm{m}<0$ in the family of knots $\operatorname{Cab}(2 \mathrm{~m}+1,2) T(3,2)$; it was 1 or $t$ before.

$$
\begin{aligned}
\mathcal{H}(q=1, t, a=0) & =1+2 t+2 t^{2}, \\
\widetilde{K} h(\operatorname{Cab}(-1,2) T(3,2))= & D A H A^{+} K h(\operatorname{Cab}(-1,2) T(3,2)) \\
=1+q^{2} t & +q^{4} t+q^{4} t^{2}+q^{6} t^{3}+q^{8} t^{3}+q^{8} t^{4} \\
& \quad+q^{10} t^{4}+2 q^{10} t^{5}+q^{14} t^{6}+q^{14} t^{7}+q^{16} t^{8} \\
& \quad+q^{16} t^{9}+q^{18} t^{10}+q^{22} t^{12}+q^{24} t^{13} .
\end{aligned}
$$

Note the use of the procedure $D A H A^{+} K h$ here and in the next example (which starts with the substitution $-1 \mapsto a / t^{2}$ in DAHA parameters).

$$
\vec{r}=\{3,-2\}, \quad \overrightarrow{\mathrm{s}}=\{2,15\}, \quad \mathcal{T}=\operatorname{Cab}(-3,2) T(3,2) ;
$$

$\mathcal{H}_{\overrightarrow{\mathrm{r}}, \overrightarrow{\mathrm{s}}}(\square ; q, t, a)=1+2 q t+q t^{2}+2 q^{2} t^{2}-q t^{3}+q^{2} t^{3}+2 q^{3} t^{3}+q^{4} t^{4}$

$$
\begin{aligned}
& +a^{3}\left(q^{4}+\frac{1}{t^{2}} q^{2}+\frac{1}{t} q^{3}+q^{5} t+q^{6} t^{2}\right) \\
& +a^{2}\left(2 q^{2}+2 q^{3}+\frac{1}{t^{2}} q+\frac{1}{t} q+\frac{1}{t} 2 q^{2}+q^{2} t+2 q^{3} t+2 q^{4} t\right. \\
& \left.+q^{3} t^{2}+2 q^{4} t^{2}+2 q^{5} t^{2}+q^{5} t^{3}+q^{6} t^{3}\right)
\end{aligned}
$$

$D A H A^{+} K h(C a b(-3,2) T(3,2))=1+q^{2} t+q^{8} t+q^{4} t^{2}+q^{6} t^{3}+q^{8} t^{4}$

$$
\begin{array}{r}
+q^{10} t^{5}+q^{12} t^{6}+q^{14} t^{6}+q^{12} t^{7} \\
+2 q^{14} t^{7}+q^{18} t^{8}+q^{18} t^{9}+q^{20} t^{10} \\
+q^{20} t^{11}+q^{22} t^{12}+q^{26} t^{14}+q^{28} t^{15} .
\end{array}
$$


Here, $\widetilde{K h}-D A H A^{+} K h=q^{12}\left(t^{5}-t^{7}\right)$. Note the use of $D A H A^{+}$here and that there is only one negative term in $\mathcal{H}(\square ; q, t, a)$, namely $-q t^{3}$. Using the procedure $D A H A^{\prime} K h$ would result in the extra term $-q^{8}\left(t-t^{3}\right)$ in this difference, where -1 does not agree with the (topological) $\partial_{2}$.

$$
\overrightarrow{\mathrm{r}}=\{3,-2\}, \quad \overrightarrow{\mathrm{s}}=\{2,17\}, \quad \mathcal{T}=\operatorname{Cab}(-5,2) T(3,2) ;
$$

$\mathcal{H}_{\vec{r}, \vec{s}}(\square ; q, t, a)=1+2 q t+q t^{2}+2 q^{2} t^{2}+q^{2} t^{3}+2 q^{3} t^{3}+q^{3} t^{4}+2 q^{4} t^{4}+q^{5} t^{5}$

$$
\begin{aligned}
& +a^{3}\left(q^{4}+\frac{1}{t^{2}} q^{2}+\frac{1}{t} q^{3}+q^{5} t+q^{6} t^{2}+q^{7} t^{3}\right) \\
& +a^{2}\left(2 q^{2}+2 q^{3}+\frac{1}{t^{2}} q+\frac{1}{t} q+\frac{1}{t} 2 q^{2}+q^{2} t+2 q^{3} t+2 q^{4} t+q^{3} t^{2}\right. \\
& \left.+2 q^{4} t^{2}+2 q^{5} t^{2}+q^{4} t^{3}+2 q^{5} t^{3}+2 q^{6} t^{3}+q^{6} t^{4}+q^{7} t^{4}\right) \\
& +a\left(3 q+q^{2}+\frac{1}{t} 1+\frac{1}{t} q+q t+4 q^{2} t+q^{3} t+2 q^{2} t^{2}+4 q^{3} t^{2}+q^{4} t^{2}+q^{2} t^{3}\right. \\
& \left.+2 q^{3} t^{3}+4 q^{4} t^{3}+q^{5} t^{3}+q^{4} t^{4}+3 q^{5} t^{4}+q^{6} t^{4}+q^{6} t^{5}\right) .
\end{aligned}
$$

$$
\mathcal{H}(q=1, t, a=0)=1+2 t+3 t^{2}+3 t^{3}+3 t^{4}+t^{5},
$$

$D A H A^{\prime} K h(C a b(-5,2) T(3,2))=1+q^{2} t+q^{4} t^{2}+q^{6} t^{3}+q^{8} t^{4}+q^{10} t^{5}$

$$
\begin{gathered}
+q^{12} t^{6}+q^{12} t^{7}+q^{14} t^{7}+q^{16} t^{8}+q^{18} t^{8} \\
+q^{16} t^{9}+2 q^{18} t^{9}+q^{22} t^{10}+q^{22} t^{11}+q^{24} t^{12} \\
+q^{24} t^{13}+q^{26} t^{14}+q^{30} t^{16}+q^{32} t^{17} .
\end{gathered}
$$

One has that $\widetilde{K} h-D A H A^{\prime} K h=q^{12}\left(t^{5}-t^{7}\right)+q^{16}\left(t^{7}-t^{9}\right)$, matching our expectations, though this is obviously not in "top" $t$-degrees.

This knot is pseudoalgebraic, as well as all further knots in the considered family. However, they are quite different numerically from the patterns in (3-11) and (3-12). For example, the polynomial $\mathcal{H}(q=1, t, a=0)$ becomes $1+2 t+3 t^{2}+3 t^{3}+4 t^{4}+3 t^{5}+t^{6}$ for $\operatorname{Cab}(-7,2) T(3,2)$. Generally,

$$
\begin{aligned}
\mathcal{H}_{\{3,-2\},\{2,2 n+7\}}(q=1, t, a=0)=1+2 t & +3 t^{2}+3 t^{3} \\
& +4\left(t^{4}+\cdots+t^{n-2}\right)+3 t^{n-1}+t^{n}
\end{aligned}
$$

for $C a b(-2 n+5,2) T(3,2), n \geq 5$. Let us provide a pseudoalgebraic knot of type (4) (for $T(4,3))$.

$$
\vec{r}=\{4,-2\}, \quad \vec{s}=\{3,7\}, \quad \mathcal{T}=\operatorname{Cab}(17,2) T(4,3) ;
$$

$\mathcal{H}_{\overrightarrow{\mathrm{r}}, \overrightarrow{\mathrm{s}}}(\square ; q, t, a)=1+q t+q^{2} t+q^{3} t+q^{4} t+q^{5} t+q^{2} t^{2}+q^{3} t^{2}+2 q^{4} t^{2}+2 q^{5} t^{2}+3 q^{6} t^{2}+q^{7} t^{2}$

$$
\begin{array}{r}
+q^{8} t^{2}+q^{3} t^{3}+q^{4} t^{3}+2 q^{5} t^{3}+3 q^{6} t^{3}+4 q^{7} t^{3}+3 q^{8} t^{3}+2 q^{9} t^{3}+q^{4} t^{4}+q^{5} t^{4}+2 q^{6} t^{4} \\
+3 q^{7} t^{4}+5 q^{8} t^{4}+4 q^{9} t^{4}+3 q^{10} t^{4}+q^{5} t^{5}+q^{6} t^{5}+2 q^{7} t^{5}+3 q^{8} t^{5}+5 q^{9} t^{5}+4 q^{10} t^{5} \\
+2 q^{11} t^{5}+q^{6} t^{6}+q^{7} t^{6}+2 q^{8} t^{6}+3 q^{9} t^{6}+5 q^{10} t^{6}+3 q^{11} t^{6}+q^{12} t^{6}+q^{7} t^{7}+q^{8} t^{7}+2 q^{9} t^{7}
\end{array}
$$




$$
\begin{array}{r}
+3 q^{10} t^{7}+4 q^{11} t^{7}+q^{12} t^{7}+q^{8} t^{8}+q^{9} t^{8}+2 q^{10} t^{8}+3 q^{11} t^{8}+3 q^{12} t^{8}+q^{9} t^{9}+q^{10} t^{9} \\
+2 q^{11} t^{9}+2 q^{12} t^{9}+q^{13} t^{9}+q^{10} t^{10}+q^{11} t^{10}+2 q^{12} t^{10}+q^{13} t^{10}+q^{11} t^{11}+q^{12} t^{11} \\
+q^{13} t^{11}+q^{12} t^{12}+q^{13} t^{12}+q^{13} t^{13}+q^{14} t^{14}
\end{array}
$$

$$
+a^{4}\left(q^{10}+q^{11}+q^{12}+q^{11} t+2 q^{12} t+q^{13} t+q^{12} t^{2}+2 q^{13} t^{2}+q^{14} t^{2}+q^{13} t^{3}+q^{14} t^{3}+q^{14} t^{4}\right)
$$$$
+a^{3}\left(q^{6}+q^{7}+2 q^{8}+2 q^{9}+2 q^{10}+q^{11}+q^{7} t+2 q^{8} t+4 q^{9} t+5 q^{10} t+5 q^{11} t+2 q^{12} t+q^{8} t^{2}\right.
$$$$
+2 q^{9} t^{2}+5 q^{10} t^{2}+7 q^{11} t^{2}+7 q^{12} t^{2}+2 q^{13} t^{2}+q^{9} t^{3}+2 q^{10} t^{3}+5 q^{11} t^{3}+7 q^{12} t^{3}+5 q^{13} t^{3}
$$

$$
\begin{array}{r}
+q^{14} t^{3}+q^{10} t^{4}+2 q^{11} t^{4}+5 q^{12} t^{4}+ \\
5 q^{13} t^{4}+2 q^{14} t^{4}+q^{11} t^{5}+2 q^{12} t^{5}+4 q^{13} t^{5}+2 q^{14} t^{5} \\
\left.+q^{12} t^{6}+2 q^{13} t^{6}+2 q^{14} t^{6}+q^{13} t^{7}+q^{14} t^{7}+q^{14} t^{8}\right)
\end{array}
$$

$+a^{2}\left(q^{3}+q^{4}+2 q^{5}+2 q^{6}+2 q^{7}+q^{8}+q^{9}+q^{4} t+2 q^{5} t+4 q^{6} t+6 q^{7} t+7 q^{8} t+6 q^{9} t+4 q^{10} t\right.$

$+q^{11} t+q^{5} t^{2}+2 q^{6} t^{2}+5 q^{7} t^{2}+8 q^{8} t^{2}+12 q^{9} t^{2}+11 q^{10} t^{2}+7 q^{11} t^{2}+q^{12} t^{2}+q^{6} t^{3}+2 q^{7} t^{3}$ $+5 q^{8} t^{3}+9 q^{9} t^{3}+14 q^{10} t^{3}+14 q^{11} t^{3}+7 q^{12} t^{3}+q^{13} t^{3}+q^{7} t^{4}+2 q^{8} t^{4}+5 q^{9} t^{4}+9 q^{10} t^{4}+14 q^{11} t^{4}$ $+11 q^{12} t^{4}+4 q^{13} t^{4}+q^{8} t^{5}+2 q^{9} t^{5}+5 q^{10} t^{5}+9 q^{11} t^{5}+12 q^{12} t^{5}+6 q^{13} t^{5}+q^{14} t^{5}+q^{9} t^{6}$ $+2 q^{10} t^{6}+5 q^{11} t^{6}+8 q^{12} t^{6}+7 q^{13} t^{6}+q^{14} t^{6}+q^{10} t^{7}+2 q^{11} t^{7}+5 q^{12} t^{7}+6 q^{13} t^{7}+2 q^{14} t^{7}$ $\left.+q^{11} t^{8}+2 q^{12} t^{8}+4 q^{13} t^{8}+2 q^{14} t^{8}+q^{12} t^{9}+2 q^{13} t^{9}+2 q^{14} t^{9}+q^{13} t^{10}+q^{14} t^{10}+q^{14} t^{11}\right)$ $+a\left(q+q^{2}+q^{3}+q^{4}+q^{5}+q^{2} t+2 q^{3} t+3 q^{4} t+4 q^{5} t+5 q^{6} t+3 q^{7} t+2 q^{8} t+q^{9} t+q^{3} t^{2}+2 q^{4} t^{2}\right.$ $+4 q^{5} t^{2}+6 q^{6} t^{2}+9 q^{7} t^{2}+8 q^{8} t^{2}+6 q^{9} t^{2}+2 q^{10} t^{2}+q^{4} t^{3}+2 q^{5} t^{3}+4 q^{6} t^{3}+7 q^{7} t^{3}+11 q^{8} t^{3}$ $+12 q^{9} t^{3}+10 q^{10} t^{3}+3 q^{11} t^{3}+q^{5} t^{4}+2 q^{6} t^{4}+4 q^{7} t^{4}+7 q^{8} t^{4}+12 q^{9} t^{4}+13 q^{10} t^{4}+10 q^{11} t^{4}$ $+2 q^{12} t^{4}+q^{6} t^{5}+2 q^{7} t^{5}+4 q^{8} t^{5}+7 q^{9} t^{5}+12 q^{10} t^{5}+12 q^{11} t^{5}+6 q^{12} t^{5}+q^{13} t^{5}+q^{7} t^{6}+2 q^{8} t^{6}$ $+4 q^{9} t^{6}+7 q^{10} t^{6}+11 q^{11} t^{6}+8 q^{12} t^{6}+2 q^{13} t^{6}+q^{8} t^{7}+2 q^{9} t^{7}+4 q^{10} t^{7}+7 q^{11} t^{7}+9 q^{12} t^{7}$ $+3 q^{13} t^{7}+q^{9} t^{8}+2 q^{10} t^{8}+4 q^{11} t^{8}+6 q^{12} t^{8}+5 q^{13} t^{8}+q^{10} t^{9}+2 q^{11} t^{9}+4 q^{12} t^{9}+4 q^{13} t^{9}$ $+q^{14} t^{9}+q^{11} t^{10}+2 q^{12} t^{10}+3 q^{13} t^{10}+q^{14} t^{10}+q^{12} t^{11}+2 q^{13} t^{11}+q^{14} t^{11}+q^{13} t^{12}$ $\left.+q^{14} t^{12}+q^{14} t^{13}\right)$.

The corresponding Khovanov polynomial equals

$D A H A^{\prime} K h(C a b(17,2) T(4,3))=1+q^{4} t^{2}+q^{6} t^{3}+q^{6} t^{4}+q^{10} t^{5}+q^{8} t^{6}+q^{12} t^{7}$

$$
\begin{array}{r}
+q^{10} t^{8}+q^{12} t^{8}+2 q^{14} t^{9}+q^{12} t^{10}+q^{14} t^{10}+2 q^{16} t^{11}+q^{18} t^{11}+3 q^{16} t^{12} \\
+q^{20} t^{12}+2 q^{18} t^{13}+2 q^{20} t^{13}+q^{18} t^{14}+q^{20} t^{14}+q^{22} t^{14}+4 q^{22} t^{15} \\
+q^{20} t^{16}+q^{22} t^{16}+q^{24} t^{16}+q^{26} t^{16}+2 q^{24} t^{17}+q^{26} t^{17}+q^{24} t^{18}+q^{28} t^{18} \\
+q^{30} t^{20}+q^{26} t^{19}+q^{28} t^{19}+q^{28} t^{20}+2 q^{30} t^{21}+q^{32} t^{22}+q^{36} t^{24}+q^{34} t^{25} .
\end{array}
$$

Here, $\widetilde{K} h-D A H A^{\prime} K h=q^{34}\left(t^{23}-t^{25}\right)$, which agrees with part (ii) of Conjecture 2.4. The corresponding (reduced and tilde-normalized) HOMFLYPT polynomial coincides with $\mathcal{H}(\square ; q, q,-a)$, as well as in all examples we considered (not only those posted in the paper). 
The last example will be a non-pseudoalgebraic knot with a relatively involved relation between $D A H A^{\prime} K h$ and $\widetilde{K} h$.

$$
\overrightarrow{\mathrm{r}}=\{4,-2\}, \quad \overrightarrow{\mathrm{s}}=\{3,17\}, \quad \mathcal{T}=\operatorname{Cab}(7,2) T(4,3)
$$

$\mathcal{H}_{\overrightarrow{\mathrm{r}}, \overrightarrow{\mathrm{s}}}(\square ; q, t, a)=-q^{2}-2 q^{3} t-q^{4} t-q^{3} t^{2}-4 q^{4} t^{2}-q^{5} t^{2}-2 q^{4} t^{3}-6 q^{5} t^{3}-q^{6} t^{3}+t^{4}-q^{4} t^{4}$ $-4 q^{5} t^{4}-7 q^{6} t^{4}-q^{7} t^{4}+q t^{5}+q^{2} t^{5}+q^{3} t^{5}+q^{4} t^{5}-q^{5} t^{5}-5 q^{6} t^{5}-7 q^{7} t^{5}-q^{8} t^{5}+q^{2} t^{6}+q^{3} t^{6}$ $+2 q^{4} t^{6}+q^{5} t^{6}-q^{6} t^{6}-4 q^{7} t^{6}-6 q^{8} t^{6}-q^{9} t^{6}+q^{3} t^{7}+q^{4} t^{7}+2 q^{5} t^{7}+q^{6} t^{7}-q^{7} t^{7}-2 q^{8} t^{7}$ $-4 q^{9} t^{7}-q^{10} t^{7}+q^{4} t^{8}+q^{5} t^{8}+q^{6} t^{8}-q^{9} t^{8}-2 q^{10} t^{8}+q^{5} t^{9}+q^{6} t^{9}-q^{11} t^{9}+q^{6} t^{10}+q^{7} t^{11}$ $+a^{5}\left(-q^{11}-\frac{1}{t^{3}} q^{8}-\frac{1}{t^{2}} q^{9}-\frac{1}{t} q^{10}-q^{12} t-q^{13} t^{2}-q^{14} t^{3}\right)$ $+a^{4}\left(-q^{7}-3 q^{8}-3 q^{9}-2 q^{10}-\frac{1}{t^{3}} q^{6}-\frac{1}{t^{3}} q^{7}-\frac{1}{t^{2}} q^{6}-\frac{1}{t^{2}} 2 q^{7}-\frac{1}{t^{2}} 2 q^{8}-\frac{1}{t} 2 q^{7}-\frac{1}{t} 3 q^{8}-\frac{1}{t} 2 q^{9}\right.$ $-2 q^{8} t-3 q^{9} t-3 q^{10} t-2 q^{11} t-q^{8} t^{2}-2 q^{9} t^{2}-3 q^{10} t^{2}-3 q^{11} t^{2}-2 q^{12} t^{2}-q^{9} t^{3}-2 q^{10} t^{3}$ $\left.-3 q^{11} t^{3}-3 q^{12} t^{3}-2 q^{13} t^{3}-q^{11} t^{4}-2 q^{12} t^{4}-2 q^{13} t^{4}-q^{14} t^{4}-q^{13} t^{5}-q^{14} t^{5}\right)$ $+a^{3}\left(-q^{5}-7 q^{6}-8 q^{7}-6 q^{8}-q^{9}-\frac{1}{t^{3}} q^{5}-\frac{1}{t^{2}} q^{4}-\frac{1}{t^{2}} 2 q^{5}-\frac{1}{t^{2}} 3 q^{6}-\frac{1}{t^{2}} q^{7}-\frac{1}{t} 3 q^{5}-\frac{1}{t} 5 q^{6}-\frac{1}{t} 5 q^{7}\right.$ $-\frac{1}{t} q^{8}-3 q^{6} t-11 q^{7} t-9 q^{8} t-6 q^{9} t-q^{10} t-q^{6} t^{2}-6 q^{7} t^{2}-13 q^{8} t^{2}-9 q^{9} t^{2}-6 q^{10} t^{2}-q^{11} t^{2}$ $-3 q^{7} t^{3}-8 q^{8} t^{3}-13 q^{9} t^{3}-9 q^{10} t^{3}-6 q^{11} t^{3}-q^{12} t^{3}+q^{6} t^{4}-3 q^{8} t^{4}-6 q^{9} t^{4}-11 q^{10} t^{4}$ $-8 q^{11} t^{4}-5 q^{12} t^{4}-q^{13} t^{4}+q^{7} t^{5}-q^{9} t^{5}-3 q^{10} t^{5}-7 q^{11} t^{5}-5 q^{12} t^{5}-3 q^{13} t^{5}-q^{11} t^{6}$ $\left.-3 q^{12} t^{6}-2 q^{13} t^{6}-q^{14} t^{6}-q^{13} t^{7}\right)$ $+a^{2}\left(-3 q^{4}-10 q^{5}-7 q^{6}-3 q^{7}-\frac{1}{t^{2}} q^{3}-\frac{1}{t^{2}} q^{4}-\frac{1}{t^{2}} q^{5}-\frac{1}{t} q^{3}-\frac{1}{t} 4 q^{4}-\frac{1}{t} 4 q^{5}-\frac{1}{t} 2 q^{6}-q^{4} t-8 q^{5} t\right.$ $-16 q^{6} t-9 q^{7} t-3 q^{8} t-3 q^{5} t^{2}-14 q^{6} t^{2}-20 q^{7} t^{2}-9 q^{8} t^{2}-3 q^{9} t^{2}-q^{5} t^{3}-8 q^{6} t^{3}-19 q^{7} t^{3}$ $-21 q^{8} t^{3}-9 q^{9} t^{3}-3 q^{10} t^{3}+q^{3} t^{4}+q^{4} t^{4}+2 q^{5} t^{4}-q^{6} t^{4}-11 q^{7} t^{4}-19 q^{8} t^{4}-20 q^{9} t^{4}-9 q^{10} t^{4}$ $-3 q^{11} t^{4}+q^{4} t^{5}+2 q^{5} t^{5}+3 q^{6} t^{5}-q^{7} t^{5}-8 q^{8} t^{5}-14 q^{9} t^{5}-16 q^{10} t^{5}-7 q^{11} t^{5}-2 q^{12} t^{5}+q^{5} t^{6}$ $+2 q^{6} t^{6}+2 q^{7} t^{6}-q^{8} t^{6}-3 q^{9} t^{6}-8 q^{10} t^{6}-10 q^{11} t^{6}-4 q^{12} t^{6}-q^{13} t^{6}+q^{6} t^{7}+q^{7} t^{7}-q^{10} t^{7}$ $\left.-3 q^{11} t^{7}-4 q^{12} t^{7}-q^{13} t^{7}+q^{7} t^{8}-q^{12} t^{8}-q^{13} t^{8}\right)$ $+a\left(-3 q^{3}-4 q^{4}-2 q^{5}-\frac{1}{t} q^{2}-\frac{1}{t} q^{3}-\frac{1}{t} q^{4}-q^{3} t-7 q^{4} t-8 q^{5} t-3 q^{6} t-3 q^{4} t^{2}-13 q^{5} t^{2}-10 q^{6} t^{2}\right.$ $-3 q^{7} t^{2}-q^{4} t^{3}-7 q^{5} t^{3}-18 q^{6} t^{3}-11 q^{7} t^{3}-3 q^{8} t^{3}+q t^{4}+q^{2} t^{4}+q^{3} t^{4}+q^{4} t^{4}-2 q^{5} t^{4}-12 q^{6} t^{4}$ $-20 q^{7} t^{4}-11 q^{8} t^{4}-3 q^{9} t^{4}+q^{2} t^{5}+2 q^{3} t^{5}+3 q^{4} t^{5}+3 q^{5} t^{5}-2 q^{6} t^{5}-12 q^{7} t^{5}-18 q^{8} t^{5}-10 q^{9} t^{5}$ $-3 q^{10} t^{5}+q^{3} t^{6}+2 q^{4} t^{6}+4 q^{5} t^{6}+3 q^{6} t^{6}-2 q^{7} t^{6}-7 q^{8} t^{6}-13 q^{9} t^{6}-8 q^{10} t^{6}-2 q^{11} t^{6}+q^{4} t^{7}$ $+2 q^{5} t^{7}+3 q^{6} t^{7}+q^{7} t^{7}-q^{8} t^{7}-3 q^{9} t^{7}-7 q^{10} t^{7}-4 q^{11} t^{7}-q^{12} t^{7}+q^{5} t^{8}+2 q^{6} t^{8}+q^{7} t^{8}$ $\left.-q^{10} t^{8}-3 q^{11} t^{8}-q^{12} t^{8}+q^{6} t^{9}+q^{7} t^{9}-q^{12} t^{9}+q^{7} t^{10}\right)$.

In this example, applying the $D A H A^{\prime}$ reduction procedure goes essentially as follows. Starting with $m=0$, one cancels consecutively the pairs in the form

$$
\pm q^{x} t^{y}\left(a^{m}+a^{m+1} / t^{2}\right) \text { and } q^{x} t^{y}\left(a^{m}-a^{m+2} / t^{4}\right)
$$


in the superpolynomial $\mathcal{H}_{\{4,-2\},\{3,17\}}$. The output is

$$
\begin{array}{r}
-q^{4}+a^{4}\left(-q^{11}-\frac{1}{t^{2}} q^{8}-\frac{1}{t^{2}} q^{9}-\frac{1}{t} q^{9}-\frac{1}{t} 2 q^{10}\right)-q^{5} t+t^{2}+q t^{3}+q^{2} t^{3}+q^{3} t^{3} \\
+q^{4} t^{3}+q^{5} t^{3}+q^{4} t^{4}+q^{5} t^{4}+2 q^{6} t^{4}+q^{7} t^{5} \\
+a^{3}\left(-q^{10}-\frac{1}{t^{2}} q^{6}-\frac{1}{t^{2}} 2 q^{7}-\frac{1}{t} 2 q^{8}-\frac{1}{t} q^{9}-q^{9} t^{2}-q^{10} t^{2}-q^{11} t^{3}\right) \\
+a^{2}\left(-\frac{1}{t^{2}} q^{6}-\frac{1}{t} q^{7}-q^{6} t-q^{8} t^{2}+q^{5} t^{3}+q^{6} t^{3}+q^{7} t^{3}+q^{7} t^{4}+q^{8} t^{4}\right) \\
+a\left(-q^{5}-\frac{1}{t} q^{4}-q^{6} t+q t^{2}+q^{2} t^{3}+q^{3} t^{3}+2 q^{4} t^{3}+2 q^{5} t^{3}\right. \\
\left.+q^{6} t^{3}+q^{5} t^{4}+2 q^{6} t^{4}+4 q^{7} t^{4}+q^{8} t^{5}\right) .
\end{array}
$$

Then we substitute $a \mapsto q_{s t}^{4} t_{s t}, q \mapsto\left(q_{s t} t_{s t}\right)^{2}$ and $t \mapsto q_{s t}^{2}$ then divide by the smallest power of $q_{s t}$, and, finally, replace $-1 \mapsto 1 / t_{s t}$. Recall that $q$ and $t$ mean actually $q_{s t}$ and $t_{s t}$, respectively, in all formulas for $K h R$-polynomials. Finally,

$D A H A^{\prime} \operatorname{Kh}(\operatorname{Cab}(7,2) T(4,3))=1+q^{32} t^{22}+q^{36} t^{24}+q^{34} t^{25}+q^{4}\left(t^{2}+t^{7}\right)$

$$
\begin{array}{r}
+q^{6}\left(t^{3}+t^{4}+t^{8}\right)+q^{8}\left(t^{6}+t^{9}\right)+q^{10}\left(t^{5}+t^{8}+t^{10}\right)+q^{14}\left(2 t^{9}+t^{10}+t^{12}\right) \\
+q^{12}\left(t^{7}+t^{8}+t^{10}+t^{13}\right)+q^{20}\left(t^{12}+2 t^{13}+t^{14}\right)+q^{16}\left(2 t^{11}+2 t^{12}+t^{14}+t^{15}\right) \\
+q^{18}\left(t^{11}+2 t^{13}+2 t^{16}\right)+q^{22}\left(t^{14}+4 t^{15}+2 t^{18}\right)+q^{24}\left(t^{16}+t^{17}+t^{19}+t^{20}\right) \\
+q^{26}\left(t^{16}+t^{17}+t^{21}\right)+q^{28}\left(t^{18}+t^{21}+t^{22}\right)+q^{30}\left(t^{20}+2 t^{23}\right)
\end{array}
$$

One has

$$
\begin{aligned}
& \widetilde{K h}(C a b(7,2) T(4,3))-D A H A^{\prime} K h=q^{12} t^{11}\left(1-t^{2}\right) \\
& \quad \times\left(1+2 q^{6} t^{3}+2 q^{10} t^{5}+q^{14} t^{8}+2 q^{18} t^{10}+q^{22} t^{12}+(1+t)\left(q^{4} t+q^{12} t^{6}+q^{16} t^{8}\right)\right),
\end{aligned}
$$

where the positivity of the coefficients matches our expectations (based on our assumptions concerning $\partial_{2}$ ) and holds for all (uncolored) nonalgebraic knots we calculated so far, where $D A H A^{\prime} K h$ is taken if the $a$-leading term in $\mathcal{H}(\square ; q, t, a)$ is negative (in our examples, the $q, t$-monomials there are all positive or all negative) and $D A H A^{+} K h$ otherwise. We hesitate to conjecture this in general.

\subsection{The case of $A_{1}$}

We are going to prove part (i) of the Connection conjecture in the $A_{1}$ case, ie the equality (4-10) $\quad \mathcal{H}\left(b \omega_{1} ; q, q,-q^{2}\right)=\widetilde{H O M}\left(b \omega_{1} ; q, q^{2}\right)=\widetilde{J D}^{A_{1}}\left(b \omega_{1} ; q, q\right), \quad b \in \mathbb{Z}_{+}$.

We need the simplest case of the theory of the difference shift operators; see [9, Theorem 2.4] and [12]. 
For $t_{\alpha}=q_{\alpha}^{k_{\nu_{\alpha}}}$, let $k=\left\{k_{v}=0,1\right\}$. Generally, we define

$$
\mathcal{X}_{k}=\prod_{\alpha \in R_{+}} \prod_{j=0}^{k_{\alpha}-1}\left(\left(q_{\alpha}^{j} X_{\alpha}\right)^{1 / 2}-\left(q_{\alpha}^{j} X_{\alpha}\right)^{-1 / 2}\right)
$$

Recall that $q_{\alpha}=q^{v_{\alpha}}$, where $v_{\alpha}:=(\alpha, \alpha) / 2$.

We put $H=H^{(k)}$ for elements $H$ from the double affine Hecke algebra $\mathcal{H}^{(k)}$ with the structural parameters $q$ and $t_{v}=q_{v}^{k_{v}}$, as well as their images in the corresponding polynomial representation, denoted by $\mathcal{V}^{(k)}$. Accordingly, $\tau_{ \pm}^{(k)}$ will be automorphisms of $\mathcal{H}^{(k)}$.

We set $\circ=\left(\left\{k_{v}=0\right\}\right)$. Then the operator $H^{\circ}$ is obtained from $H$ by replacing every $Y_{b}(b \in P)$ by the difference operators $b^{-1}$, and $T_{w}(w \in W)$ by $w$. One has

$$
\tau_{+}^{\circ}\left(Y_{b}\right)=q^{-(b, b) / 2} X_{b} Y_{b}, \tau_{-}^{\circ}\left(X_{b}\right)=q^{(b, b) / 2} Y_{b} X_{b} \quad \text { for } b \in P .
$$

Lemma 4.1 Let $H$ be an algebraic expression with complex coefficients in terms of the standard $W$-symmetrizations of monomials with respect to $\left\{X_{b}\right\}$ and those with respect to $\left\{Y_{b}\right\}$. The restriction of the corresponding operator to the subspace $\left(\mathcal{V}^{(k)}\right)^{W}$ of $W$-invariant elements in $\mathcal{V}^{(k)}$ will be denoted by $H_{\text {sym }}^{(k)}$. Then

$$
H_{\text {sym }}^{(k)}=\mathcal{X}_{k}^{-1} H_{\text {sym }}^{\circ} \mathcal{X}_{k}, \quad\left(\tau_{ \pm}^{(k)}\left(H^{(k)}\right)\right)_{s y m}=\mathcal{X}_{k}^{-1}\left(\tau_{ \pm}^{\circ}\left(H^{\circ}\right)\right)_{s y m} \mathcal{X}_{k}
$$

Let us formally set for $\lambda, \lambda^{\prime} \in \mathbb{C}^{n}$,

$$
X_{\lambda}=q^{x_{\lambda}}, \quad x_{\lambda}=(\lambda, x), \quad X_{\lambda+\lambda^{\prime}}=X_{\lambda} X_{\lambda^{\prime}}, \quad x^{2}=(x, x)=\sum_{i=1}^{n}\left(x, \omega_{i}\right)\left(x, \alpha_{i}^{\vee}\right) .
$$

We will treat $\tau_{ \pm}^{\circ}$ and any elements $\gamma \in \operatorname{SL}(2, \mathbb{Z})$ as automorphisms of the linear span of $X_{\lambda} q^{M x^{2} / 2}$ for all $\lambda \in \mathbb{C}^{n}$ and $M$ from a sufficiently general subset of $\mathbb{C}$ (see the inequalities for $M, N, M^{\prime}$ below). Given $\lambda$, only $c \lambda$ for certain $c \in \mathbb{C}$ will be actually needed in (4-13) and (4-14).

This linear span will be denoted by $\tilde{\mathcal{V}}$. The action of $\widehat{W}$ in $\mathcal{V}$ is naturally extended to $\tilde{\mathcal{V}}$ through its action on $\left\{x_{\lambda}\right\} ; q^{M x^{2} / 2}$ are $W$-invariant and satisfy the standard difference equations for $P \subset \widehat{W}$. 
The main formulas we need are as follows:

$$
\begin{aligned}
\sigma^{\circ}\left(X_{\lambda} q^{-M x^{2} / 2}\right) & =\frac{q^{\lambda^{2} /(2 M)}}{M^{1 / 2}} X_{\lambda / M} q^{+x^{2} /(2 M)}, \\
\left(\tau_{+}^{\circ}\right)^{N}\left(X_{\lambda} q^{-M x^{2} / 2}\right) & =X_{\lambda} q^{(N-M) x^{2} / 2} \\
\left(\tau_{-}^{\circ}\right)^{N}\left(X_{\lambda} q^{-M x^{2} / 2}\right) & =\left(\left(\sigma^{\circ}\right)^{-1}\left(\tau_{+}^{\circ}\right)^{-N} \sigma^{\circ}\right)\left(X_{\lambda} e^{-M x^{2} / 2}\right) \\
& =\frac{1}{(1-M N)^{1 / 2}} q^{\frac{\lambda^{2} N}{2(1-M N)}} X_{\lambda /(1-M N)} q^{-x^{2} \frac{M}{2(1-M N)}} .
\end{aligned}
$$

Here, $N \in \mathbb{Z}$ and the parameters $M$ are sufficiently general complex numbers; for instance, $M N \neq 1$ in the last formula. We also assume that $\left(M M^{\prime}\right)^{1 / 2}=M^{1 / 2}\left(M^{\prime}\right)^{1 / 2}$ for $M, M^{\prime} \in \mathbb{C}$.

These formulas can be readily extended to the following free action of the whole $\operatorname{SL}(2, \mathbb{Z})$ on $X_{\lambda} q^{z x^{2} / 2}$,

$$
\gamma^{\circ}\left(X_{\lambda} q^{z x^{2} / 2}\right)=\frac{1}{(c z+d)^{1 / 2}} q^{-\frac{\lambda^{2} c}{2(c z+d)}} X_{\frac{\lambda}{c z+d}} q^{\frac{a z+b}{c z+d} x^{2} / 2}
$$

for $\gamma=\left(\begin{array}{ll}a & b \\ c & d\end{array}\right) \in \operatorname{SL}(2, \mathbb{Z}), \lambda \in \mathbb{C}^{n}$, and for generic $z \in \mathbb{C}$.

DAHA-Jones polynomials We will now switch to the $A_{1}$ case. Let $\alpha=\alpha_{1}, s=s_{1}$ and $\omega=\omega_{1}$; then $\alpha=\alpha_{1}=2 \omega$ and $\rho=\omega$. The extended affine Weyl group is $\widehat{W}=\langle s, \omega\rangle$. The weights $b \omega(b \in \mathbb{Z})$ will be denoted simply by $b$.

The double affine Hecke algebra $\mathcal{H}$ is generated by invertible elements $Y=Y_{\omega}$, $T=T_{1}$, and $X=X_{\omega}$, subject to the quadratic relation $\left(T-t^{1 / 2}\right)\left(T+t^{-1 / 2}\right)=0$ and the cross relations

$$
T X T=X^{-1}, \quad T^{-1} Y T^{-1}=Y^{-1}, \quad Y^{-1} X^{-1} Y X T^{2} q^{1 / 2}=1 .
$$

The field of definition will be $\mathbb{Q}\left(q^{1 / 4}, t^{1 / 2}\right)$. Here, $q^{ \pm 1 / 4}$ is needed for the automorphisms $\tau_{ \pm}$:

$$
\begin{array}{lll}
\tau_{+}(X)=X, & \tau_{+}(T)=T, & \tau_{+}(Y)=q^{-1 / 4} X Y, \\
\tau_{-}(Y)=Y, & \tau_{-}(T)=T, & \tau_{-}(X)=q^{1 / 4} Y X .
\end{array}
$$

We will prove the Connection conjecture for the nonreduced version of the DAHA-Jones polynomials. Namely, we modify (2-12) as

$$
J D_{\overrightarrow{\mathrm{r}}, \overrightarrow{\mathrm{s}}}^{\#}(b ; q, t):=\left\{\widehat{\gamma}_{1}\left(\cdots\left(\widehat{\gamma}_{\ell-1}\left(\left(\widehat{\gamma}_{\ell}\left(P_{b}^{(k)}\right)\right) \Downarrow\right) \Downarrow\right) \cdots\right)\right\}^{(k)},
$$

for $t:=q^{k}$ and $\gamma=\gamma_{r, s}$ lifted to $\hat{\gamma} \in \operatorname{PSL}_{2}^{\wedge}(\mathbb{Z})$, where we show explicitly the dependence of the $P$-polynomial on the parameter $k$ and also use ${ }^{(k)}$ for the corresponding 
coinvariant. Generally, the nonreduced DAHA-Jones polynomials are inconvenient because of their nontrivial $q, t$-denominators. However, they become $q$-polynomials as $t=q$ and simplify the considerations.

\subsection{Jones polynomials}

We are going to use the relation for the colored Jones polynomials of the cable $C a b(a, r) K$ with that of the initial knot $K$. See $[32 ; 44]$ and references therein. Note that $t$ in [44] is our $q^{1 / 4}$, and $C a b(r, s)$ there is our $C a b(\mathrm{a}, \mathrm{r})$ (in this order!).

Assuming here and below that the $j$-summation has step 1 , one has

$$
\mathcal{J}_{C a b(\mathrm{a}, \mathrm{r}) K}^{\#}(b ; q)=q^{-\mathrm{ar} b(b+2) / 4} \sum_{j=-b / 2}^{b / 2} q^{\mathrm{aj}(j \mathrm{r}+1)} \mathcal{J}_{K}^{\#}(2 \mathrm{r} j ; q) .
$$

This formula gives a recursive definition of the Jones polynomials of iterated knots; the unknot is normalized as

$$
\mathcal{J}_{\bigcirc}^{\#}(b ; q)=\left(q^{(b+1) / 2}-q^{-(b+1) / 2}\right) /\left(q^{1 / 2}-q^{-1 / 2}\right) .
$$

Proposition 4.2 For any (possibly negative) $\{\mathrm{r}, \mathrm{s}\}$ from Theorem 2.1,

$$
\begin{aligned}
J D_{\left\{\mathrm{r}_{1}, \ldots, \mathrm{r}_{\ell}\right\},\left\{\mathrm{s}_{1}, \ldots, \mathrm{s}_{\ell}\right\}}^{\#}(b ; q, t & \mapsto q) \\
= & q^{-\mathrm{a}_{\ell} \mathrm{r}_{\ell} b(b+2) / 4} \mathcal{J}_{T\left(\left\{\mathrm{r}_{1}, \ldots, \mathrm{r}_{\ell}\right\},\left\{\mathrm{s}_{1}, \ldots, \mathrm{s}_{\ell}\right\}\right)}^{\#}\left(b ; q \mapsto q^{-1}\right),
\end{aligned}
$$

where $T\left(\left\{\mathrm{r}_{1}, \ldots, \mathrm{r}_{\ell}\right\},\left\{\mathrm{s}_{1}, \ldots, \mathrm{s}_{\ell}\right\}\right)=\operatorname{Cab}(\overrightarrow{\mathrm{a}}, \overrightarrow{\mathrm{r}})=\left(\operatorname{Cab}\left(\mathrm{a}_{\ell}, \mathrm{r}_{\ell}\right) \cdots \operatorname{Cab}\left(\mathrm{a}_{1}, \mathrm{r}_{1}\right)\right)(\bigcirc)$, and $\mathrm{a}_{i}$ are recursively defined by relations $\mathrm{a}_{i+1}=\mathrm{r}_{i} \mathrm{r}_{i+1} \mathrm{a}_{i}+\mathrm{s}_{i+1}$ for $1 \leq i \leq \ell-1$ and the initial condition $\mathrm{a}_{1}=\mathrm{s}_{1}$.

Proof As above, we formally set $X_{\lambda}=q^{\lambda x}$ for $\lambda \in \mathbb{C}$, which extends $X_{b}=X_{b \omega_{1}}=$ $X^{b}$ for $b$ in $\mathbb{Z}$ (identified with the lattice $\left.P=\mathbb{Z} \omega_{1}\right)$. The free action of $\operatorname{SL}(2, \mathbb{Z})$ on $X_{\lambda} q^{M x^{2}}$ from (4-13) will be used for $\gamma=\left(\begin{array}{c}r \star \\ s_{\star}\end{array}\right)$. We need the formula

$$
\gamma^{\circ}\left(X_{\lambda}\left(\gamma^{\circ}\right)^{-1}\left(X_{\mu}\right)\right)=q^{-r s \lambda^{2} / 4-s \lambda \mu / 2} X_{r \lambda+\mu},
$$

which results in

$$
\begin{aligned}
\gamma^{\circ}\left(P_{b}^{(1)}\left(\gamma^{\circ}\right)^{-1}\left(X-X^{-1}\right)\right) & =\gamma^{\circ}\left(\sum_{j=-b / 2}^{b / 2} X^{2 j}\left(\gamma^{\circ}\right)^{-1}\left(X-X^{-1}\right)\right) \\
& =\sum_{j=-b / 2}^{b / 2} q^{-(r j+1) s j} X^{2 r j+1}-\sum_{j=-b / 2}^{b / 2} q^{-(r j-1) s j} X^{2 r j-1}
\end{aligned}
$$




$$
\begin{aligned}
& =\sum_{j=-b / 2}^{b / 2} q^{-(r j+1) s j} X^{2 r j+1}-\sum_{j=-b / 2}^{b / 2} q^{-(r j+1) s j} X^{-2 r j-1} \\
& =\sum_{j=-b / 2}^{b / 2} q^{-(r j+1) s j}\left(X-X^{-1}\right) P_{2 r j}^{(1)},
\end{aligned}
$$

and then implies the relation

$$
\begin{aligned}
\left(\widehat{\gamma}_{\ell}^{(1)} P_{b}^{(1)}\left(\widehat{\gamma}_{\ell}^{(1)}\right)^{-1}\right) \Downarrow & =\left(\mathcal{X}_{1}^{-1} \hat{\gamma}_{\ell}^{\circ} \mathcal{X}_{1} P_{b}^{(1)} \mathcal{X}_{1}^{-1}\left(\widehat{\gamma}_{\ell}^{\circ}\right)^{-1} \mathcal{X}_{1}\right) \Downarrow \\
& =\sum_{j=-b / 2}^{b / 2} q^{-\left(r_{\ell} j+1\right) s_{\ell} j} P_{2 r_{\ell} j}^{(1)}
\end{aligned}
$$

for $\mathcal{X}_{1}=X-X^{-1}$. Thus, we can switch from $k=1$ to the case of $k=0$, where the free action of $\operatorname{PSL}(2, \mathbb{Z})$ can be used.

Using the latter relation we can proceed by induction as follows:

$$
\begin{aligned}
& J D_{T\left(\left\{\mathrm{r}_{1}, \ldots, \mathrm{r}_{\ell}\right\},\left\{\mathrm{s}_{1}, \ldots, \mathrm{s}_{\ell}\right\}\right)}^{\#}(b ; q, t \mapsto q) \\
& \left.=\left\{\widehat{\gamma}_{1}^{(1)}\left(\cdots\left(\widehat{\gamma}_{\ell-1}^{(1)}\left(\widehat{\gamma}_{\ell}^{(1)} P_{b}^{(1)}\left(\widehat{\gamma}_{\ell}^{(1)}\right)^{-1}\right) \Downarrow\left(\widehat{\gamma}_{\ell-1}^{(1)}\right)^{-1}\right) \Downarrow\right) \cdots\right)\left(\widehat{\gamma}_{1}^{(1)}\right)^{-1}\right\}^{(1)} \\
& \left.=\sum_{j=-b / 2}^{b / 2} q^{-\left(r_{\ell} j+1\right) s_{\ell} j}\left\{\hat{\gamma}_{1}^{(1)}\left(\cdots\left(\widehat{\gamma}_{\ell-1}^{(1)} P_{2 r_{\ell} j}^{(1)}\left(\widehat{\gamma}_{\ell-1}^{(1)}\right)^{-1}\right) \Downarrow\right) \cdots\right)\left(\widehat{\gamma}_{1}^{(1)}\right)^{-1}\right\}^{(1)} \\
& =\sum_{j=-b / 2}^{b / 2} q^{-\left(\mathrm{r}_{\ell} j+1\right) \mathrm{s}_{\ell} j} J D_{T\left(\left\{\mathrm{r}_{1}, \ldots, \mathrm{r}_{\ell-1}\right\},\left\{\mathrm{s}_{1}, \ldots, \mathrm{s}_{\ell-1}\right\}\right)}^{\#}\left(2 \mathrm{r}_{\ell} j ; q, t \mapsto q\right) \\
& \begin{aligned}
=\sum_{j=-b / 2}^{b / 2} q^{-\left(\mathrm{r}_{\ell} j+1\right) \mathrm{s}_{\ell} j-\mathrm{a}_{\ell-1} \mathrm{r}_{\ell-1}\left(2 \mathrm{r}_{\ell} j\right)\left(2 \mathrm{r}_{\ell} j+2\right) / 4} & \\
& \times \mathcal{J}_{T\left(\left\{\mathrm{r}_{1}, \ldots, \mathrm{r}_{\ell-1}\right\},\left\{\mathrm{s}_{1}, \ldots, \mathrm{s}_{\ell-1}\right\}\right)}^{\#}\left(2 \mathrm{r}_{\ell} j ; q \mapsto q^{-1}\right)
\end{aligned} \\
& =\sum_{j=-b / 2}^{b / 2} q^{-\left(\mathrm{r}_{\ell} j+1\right)\left(\mathrm{s}_{\ell}+\mathrm{a}_{\ell-1} \mathrm{r}_{\ell} \mathrm{r}_{\ell-1}\right) j} \mathcal{J}_{T\left(\left\{\mathrm{r}_{1}, \ldots, \mathrm{r}_{\ell-1}\right\},\left\{\mathrm{s}_{1}, \ldots, \mathrm{s}_{\ell-1}\right\}\right)}^{\#}\left(2 \mathrm{r}_{\ell} j ; q \mapsto q^{-1}\right) \\
& =\sum_{j=-b / 2}^{b / 2} q^{-\left(\mathrm{r}_{\ell} j+1\right) \mathrm{a}_{\ell} j} \mathcal{J}_{T\left(\left\{\mathrm{r}_{1}, \ldots, \mathrm{r}_{\ell-1}\right\},\left\{\mathrm{s}_{1}, \ldots, \mathrm{s}_{\ell-1}\right\}\right)}^{\#}\left(2 \mathrm{r}_{\ell} j ; q \mapsto q^{-1}\right) \\
& =q^{-\mathrm{a} \ell \mathrm{r}_{\ell} b(b+2) / 4} \mathcal{J}_{T\left(\left\{\mathrm{r}_{1}, \ldots, \mathrm{r}_{\ell}\right\},\left\{\mathrm{s}_{1}, \ldots, \mathrm{s}_{\ell}\right\}\right)}^{\#}\left(b ; q \mapsto q^{-1}\right) .
\end{aligned}
$$


Finally,

$$
J D_{\bigcirc}^{\#}(b ; q, t \mapsto q)=\frac{q^{(b+1) / 2}-q^{-(b+1) / 2}}{q^{1 / 2}-q^{-1 / 2}}=\mathcal{J}_{\bigcirc}^{\#}\left(b ; q \mapsto q^{-1}\right),
$$

which completes the proof.

\subsection{Concluding remarks}

Concerning part (iii) of the Connection conjecture, adding colors (arbitrary Young diagrams $\lambda$ ) to [33, Conjecture 1.2] is a natural challenge. This would provide a $t$-extension of [30, Theorem 1.2]. Then, our construction (which is for arbitrary colors) will be employed at full potential; we hope to extend it from iterated torus knots (the setup of this paper) to iterated links.

The switch to the curves $C_{\lambda}$ from [30, Section 1.3] supported on $C=\mathcal{C}_{\overrightarrow{\mathrm{r}}, \overrightarrow{\mathrm{s}}}$ and associated with Young diagrams $\lambda$ is natural here. This approach was initiated in [14] (via the so called resolved conifold). Using (the germs of) such curves can be hopefully combined with considering the weight filtration in the cohomology of the corresponding nested Hilbert schemes and Jacobian factors.

On the other hand, DAHA uniformly manages arbitrary root systems and weights via the Macdonald polynomials; changing the curves $\mathcal{C}_{\vec{r}, \vec{s}}$ for incorporating the colors does not seem really necessary from this perspective. Instead, the spaces $\operatorname{Bun}_{G}\left(\mathcal{C}_{\vec{r}, \vec{s}}\right)$ of $G$-bundles over $\mathcal{C}_{\vec{r}, \vec{s}}$ for the simple Lie group $G$ associated with the root system $R$ can be expected. This is related to the interpretation of the Jacobian factors as Springer fibers due to [29]; $\mathcal{C}_{\vec{r}, \vec{s}}$ would then become spectral curves.

See also [8, Corollary 2.3] (and Theorem 4.10), where this connection was established for torsion free sheaves over arbitrary (possibly singular) coverings of $P^{1}$ or elliptic curves $E$, assuming the semistability (stability for $E$ ) of their direct images, the sheaves over $P^{1}$ or $E$. The connection with Baker functions and the "group" $\tau-$ function was established there (Proposition 2.4), which makes this construction a variant of the Hitchin system for factorizable Lie group schemes over $E$ or $P^{1}$. These schemes are associated with arbitrary (nonunitary) classical $r$-matrices; see [8] and [10, Section 1.7].

This approach potentially leads to certain cell decompositions of Jacobian factors, different from those in [36] and defined via the Kac-Moody groups. The latter act as Hecke transformations in the fibers of the coverings above; the output, but not the procedure, is entirely local (only the germ $\mathcal{C}$ is really necessary). 
Khovanov-Rozansky homology On the topological side, the skein relations, the key in [30, Theorem 1.2], are missing (by now) upon the switch from the HOMFLYPT polynomials to their homology from $[27 ; 28 ; 26 ; 48 ; 39]$ (generalizing the Khovanov homology for the Jones polynomials). Adding colors (dominant weights) here is generally a difficult problem, though the categorification theory (based on Quantum Groups) provides tools for this.

In contrast to the Khovanov polynomials $(n=2)$, not many formulas for KhovanovRozansky polynomials $K h R_{\text {stab }}$ are known. Let us mention some recent progress here (by Oblomkov and Rozansky) based on further development of the theory of matrix factorization. This may provide much better tools for finding the $K h R$-polynomials (at least for torus knots and in the uncolored case).

Recall that Khovanov-Rozansky polynomials are defined as the Poincaré polynomials of the corresponding triply graded homology. They exist for any links; such an universality is one of the reasons why their theory is so complicated. For us, the KhovanovRozansky and Khovanov polynomials are the only way so far to interpret geometrically (topologically) the DAHA superpolynomials of arbitrary iterated torus knots. They are generally nonalgebraic and their connection with the algebraic geometry is not clear at the moment, though there is some partial progress with finding the geometric meaning of pseudoalgebraic knots.

As the examples of Section 4.1 indicate, it is possible that part (ii) of Conjecture 2.4 can be extended to any (uncolored) non-pseudoalgebraic knots using a special procedure of replacing the negative terms in the DAHA superpolynomials by certain positive ones. Such knots are defined exactly as those with some negative terms. Algebraically this procedure seems somewhat artificial, but it worked for all non-pseudoalgebraic knots we considered upon the reduction from superpolynomials to the Khovanov polynomials (where we can compare our polynomials vs. the topological ones). However, the evidence is insufficient so far.

Betti numbers The examples above confirm our conjecture that the DAHA superpolynomials split the Euler numbers of the Jacobian factors (compactified Jacobians) of unibranch plane curve singularities into the corresponding Betti numbers. Recall that this is under the substitution $a \mapsto 0$ and $q \mapsto 1$ in DAHA superpolynomials. See (2-22) concerning $q=1$, the case of trivial central charge.

Such a specialization is the simplest demonstration of the power of adding " $t$ " to the theory, the refinement; our conjecture on Betti numbers is presumably related to the expected connection of our superpolynomials to $\mathscr{P}_{\text {alg }}$, which is a reduced modification 
of that from [33, Conjecture 2]:

$$
\mathscr{P}_{\mathrm{alg}}=\left(\frac{q_{s t}}{a_{s t}}\right)^{\mu} \frac{1-q_{s t}^{2}}{1+a_{s t}^{2} t_{s t}} \sum_{l, m \geq 0} q_{s t}^{2 l} a_{s t}^{2 m} t_{s t}^{m^{2}} \mathfrak{w}\left(\mathcal{C}_{\overrightarrow{\mathrm{r}}, \overrightarrow{\mathrm{s}}}^{[l \leq l+m]}\right) .
$$

Here, $\mu$ is the Milnor number ( $\mu=2 \delta$ in the unibranch case) and $\mathfrak{w}$ is the weight filtration in the compactly supported cohomology of the corresponding scheme; its existence was justified by Deligne. See the Overview and [33, Section 4]. The parameter $t_{s t}$ is associated to it; $t_{s t}^{m^{2}}$ there is a necessary normalization.

The spectral sequence $E_{1}$ in terms of the weight filtration on the compactly supported cohomology over $\mathbb{Q}$, which converges to the whole cohomology and degenerates at $E_{2}$ (for smooth not necessarily compact manifolds), can potentially connect (4-24) with the Betti numbers. Here, one needs to know that the odd cohomology of the Jacobian factors vanish for unibranch plane curve singularities (the van Straten-Warmt conjecture) and that the corresponding mixed Hodge filtration is pure (a conjecture). We thank V Shende for the explanations; see [33] (though the Betti numbers are not explicitly discussed there).

Generally, not too much is known concerning the Betti numbers of the general Jacobian factors, though they were calculated for many torus knots and those for the series $(4,2 u, v)$ of Puiseux exponents, where $4<2 u<v$ for both $u$ and $v$ odd. See [36] and above. Actually there is not even a general conjectural formula for them in the case of arbitrary torus knots, which can be potentially produced in our approach (to be considered elsewhere).

We checked quite a few examples (beyond those posted above), including torus knots. For instance, our DAHA-Betti polynomial for $T(12,7)$ is

$$
\begin{array}{r}
\mathcal{H}_{12,7}(\square ; q=1, t, a=0)=1+6 t+19 t^{2}+41 t^{3}+70 t^{4}+102 t^{5}+133 t^{6}+159 t^{7} \\
+178 t^{8}+190 t^{9}+194 t^{10}+193 t^{11}+184 t^{12}+174 t^{13}+159 t^{14}+145 t^{15} \\
+127 t^{16}+112 t^{17}+95 t^{18}+81 t^{19}+66 t^{20}+55 t^{21}+43 t^{22}+35 t^{23} \\
+26 t^{24}+20 t^{25}+14 t^{26}+11 t^{27}+7 t^{28}+5 t^{29}+3 t^{30}+2 t^{31}+t^{32}+t^{33}
\end{array}
$$

Its coefficients match those given before [36, Theorem 21]. We will omit the corresponding DAHA superpolynomial; note that it can be also obtained by E Gorsky's construction (via rational DAHA).

Semigroups and semimodules Apart from the torus knots, we established the coincidence of the DAHA-Betti numbers with the actual ones for many entries in the table before [36, Conjecture 23]. The examples in this table are more involved that those 
for torus knots, since not all modules $\Delta$ over the valuation semigroup $\Gamma$ contribute to the cell decompositions of $J\left(\mathcal{C}_{\vec{r}, \vec{s}}\right)$ from [36]. See Definition 8 of admissible modules there, as well as Theorem 13 (we use his notation). Let us provide an example, which demonstrates how surprising our conjectural connection with DAHA is and the challenges that we face.

The set of Puiseux exponents $(4,2 u, v)=(4,14,17)$ corresponds to the Newton's pairs $\left\{r_{1}=7, s_{1}=2\right\}$ and $\left\{r_{2}=2, s_{2}=3\right\}$. They encode the planar singularity

$$
\mathcal{C}_{\overrightarrow{\mathrm{r}}, \overrightarrow{\mathrm{s}}}: y=x^{\mathrm{s}_{1} / \mathrm{r}_{1}}\left(c+x^{\mathrm{s}_{2} /\left(\mathrm{r}_{1} \mathrm{r}_{2}\right)}\right)=x^{2 / 7}\left(c+x^{3 / 14}\right) \quad \text { with } \mathcal{R}=\mathbb{C} \llbracket z^{4}, z^{14}+z^{17} \rrbracket .
$$

Recall that the latter is the local ring of this singularity, and its topological link is $\operatorname{Cab}(31,2) T(7,2)$.

The semigroup is $\Gamma=\langle 4,14,31\rangle$ (41 is the last missing valuation for this $\mathcal{C}$ ); $\delta=21$ and $e\left(J\left(\mathcal{C}_{\vec{r}, \vec{s}}\right)\right)=194$. The total number of semimodules $\Delta$ (which is relatively simple to calculate) is 222 . Thus, not all contribute to the cell decomposition; 28 are not present. Their list is involved combinatorially, as well as the calculation of the dimensions of the cells corresponding to admissible $\Delta$ (though there are explicit formulas for the dimensions and the admissibility). It is not impossible that semimodules $\Delta$ have some connection to the DAHA-procedure. However DAHA also produces the correct number of missing (nonadmissible) ones, which seems more surprising (at the moment).

The corresponding $\gamma$-matrices, needed in the construction of the DAHA superpolynomial, are $\gamma_{7,2}=\tau_{+}^{3} \tau_{-}^{2}$ and $\gamma_{2,3}=\tau_{-} \tau_{+} \tau_{-}$. Note the order of 2,3 in the last $\gamma$. Its Betti-reduction $\mathcal{H}_{\{7,2\},\{2,3\}}(\square ; q=1, t, a=0)$ matches the corresponding entry in [36]. It reads

$$
\begin{aligned}
1 & +3 t+6 t^{2}+10 t^{3}+13 t^{4}+15 t^{5}+16 t^{6}+16 t^{7}+16 t^{8}+16 t^{9}+15 t^{10} \\
& +14 t^{11}+12 t^{12}+10 t^{13}+8 t^{14}+7 t^{15}+5 t^{16}+4 t^{17}+3 t^{18}+2 t^{19}+t^{20}+t^{21} .
\end{aligned}
$$

For the sake of completeness, let us provide the whole DAHA superpolynomial. Its $a$-degree is $\mathrm{s}_{1} \mathrm{r}_{2}-1=3$;

$$
\vec{r}=\{7,2\}, \quad \vec{s}=\{2,3\}, \quad \mathcal{T}=\operatorname{Cab}(31,2) T(7,2) ;
$$

$$
\begin{aligned}
& \mathcal{H}_{\{7,2\},\{2,3\}}(\square ; q, t, a)=1+q t+q^{2} t+q^{3} t+q^{2} t^{2}+q^{3} t^{2}+2 q^{4} t^{2}+q^{5} t^{2}+q^{6} t^{2}+q^{3} t^{3}+q^{4} t^{3} \\
&+ 2 q^{5} t^{3}+2 q^{6} t^{3}+2 q^{7} t^{3}+q^{8} t^{3}+q^{9} t^{3}+q^{4} t^{4}+q^{5} t^{4}+2 q^{6} t^{4}+2 q^{7} t^{4}+3 q^{8} t^{4}+2 q^{9} t^{4} \\
&+ 2 q^{10} t^{4}+q^{5} t^{5}+q^{6} t^{5}+2 q^{7} t^{5}+2 q^{8} t^{5}+3 q^{9} t^{5}+3 q^{10} t^{5}+3 q^{11} t^{5}+q^{6} t^{6}+q^{7} t^{6}+2 q^{8} t^{6} \\
&+ 2 q^{9} t^{6}+3 q^{10} t^{6}+3 q^{11} t^{6}+4 q^{12} t^{6}+q^{7} t^{7}+q^{8} t^{7}+2 q^{9} t^{7}+2 q^{10} t^{7}+3 q^{11} t^{7}+3 q^{12} t^{7} \\
&+4 q^{13} t^{7}+q^{8} t^{8}+q^{9} t^{8}+2 q^{10} t^{8}+2 q^{11} t^{8}+3 q^{12} t^{8}+3 q^{13} t^{8}+4 q^{14} t^{8}+q^{9} t^{9}+q^{10} t^{9} \\
&+2 q^{11} t^{9}+2 q^{12} t^{9}+3 q^{13} t^{9}+3 q^{14} t^{9}+4 q^{15} t^{9}+q^{10} t^{10}+q^{11} t^{10}+2 q^{12} t^{10}+2 q^{13} t^{10}
\end{aligned}
$$


$+3 q^{14} t^{10}+3 q^{15} t^{10}+3 q^{16} t^{10}+q^{11} t^{11}+q^{12} t^{11}+2 q^{13} t^{11}+2 q^{14} t^{11}+3 q^{15} t^{11}+3 q^{16} t^{11}$

$+2 q^{17} t^{11}+q^{12} t^{12}+q^{13} t^{12}+2 q^{14} t^{12}+2 q^{15} t^{12}+3 q^{16} t^{12}+2 q^{17} t^{12}+q^{18} t^{12}+q^{13} t^{13}$

$+q^{14} t^{13}+2 q^{15} t^{13}+2 q^{16} t^{13}+3 q^{17} t^{13}+q^{18} t^{13}+q^{14} t^{14}+q^{15} t^{14}+2 q^{16} t^{14}+2 q^{17} t^{14}$ $+2 q^{18} t^{14}+q^{15} t^{15}+q^{16} t^{15}+2 q^{17} t^{15}+2 q^{18} t^{15}+q^{19} t^{15}+q^{16} t^{16}+q^{17} t^{16}+2 q^{18} t^{16}+q^{19} t^{16}$

$+q^{17} t^{17}+q^{18} t^{17}+2 q^{19} t^{17}+q^{18} t^{18}+q^{19} t^{18}+q^{20} t^{18}+q^{19} t^{19}+q^{20} t^{19}+q^{20} t^{20}+q^{21} t^{21}$ $+a^{3}\left(q^{6}+q^{7} t+q^{8} t+q^{9} t+q^{8} t^{2}+q^{9} t^{2}+2 q^{10} t^{2}+q^{11} t^{2}+q^{12} t^{2}+q^{9} t^{3}+q^{10} t^{3}+2 q^{11} t^{3}\right.$

$+2 q^{12} t^{3}+2 q^{13} t^{3}+q^{10} t^{4}+q^{11} t^{4}+2 q^{12} t^{4}+2 q^{13} t^{4}+3 q^{14} t^{4}+q^{11} t^{5}+q^{12} t^{5}+2 q^{13} t^{5}$ $+2 q^{14} t^{5}+3 q^{15} t^{5}+q^{12} t^{6}+q^{13} t^{6}+2 q^{14} t^{6}+2 q^{15} t^{6}+3 q^{16} t^{6}+q^{13} t^{7}+q^{14} t^{7}+2 q^{15} t^{7}$ $+2 q^{16} t^{7}+3 q^{17} t^{7}+q^{14} t^{8}+q^{15} t^{8}+2 q^{16} t^{8}+2 q^{17} t^{8}+2 q^{18} t^{8}+q^{15} t^{9}+q^{16} t^{9}+2 q^{17} t^{9}$ $+2 q^{18} t^{9}+q^{19} t^{9}+q^{16} t^{10}+q^{17} t^{10}+2 q^{18} t^{10}+q^{19} t^{10}+q^{17} t^{11}+q^{18} t^{11}+2 q^{19} t^{11}$ $\left.+q^{18} t^{12}+q^{19} t^{12}+q^{20} t^{12}+q^{19} t^{13}+q^{20} t^{13}+q^{20} t^{14}+q^{21} t^{15}\right)$ $+a^{2}\left(q^{3}+q^{4}+q^{5}+q^{4} t+2 q^{5} t+3 q^{6} t+2 q^{7} t+q^{8} t+q^{5} t^{2}+2 q^{6} t^{2}+4 q^{7} t^{2}+4 q^{8} t^{2}+4 q^{9} t^{2}\right.$ $+2 q^{10} t^{2}+q^{11} t^{2}+q^{6} t^{3}+2 q^{7} t^{3}+4 q^{8} t^{3}+5 q^{9} t^{3}+6 q^{10} t^{3}+5 q^{11} t^{3}+3 q^{12} t^{3}+q^{7} t^{4}+2 q^{8} t^{4}$ $+4 q^{9} t^{4}+5 q^{10} t^{4}+7 q^{11} t^{4}+7 q^{12} t^{4}+5 q^{13} t^{4}+q^{8} t^{5}+2 q^{9} t^{5}+4 q^{10} t^{5}+5 q^{11} t^{5}+7 q^{12} t^{5}$ $+8 q^{13} t^{5}+6 q^{14} t^{5}+q^{9} t^{6}+2 q^{10} t^{6}+4 q^{11} t^{6}+5 q^{12} t^{6}+7 q^{13} t^{6}+8 q^{14} t^{6}+6 q^{15} t^{6}+q^{10} t^{7}$ $+2 q^{11} t^{7}+4 q^{12} t^{7}+5 q^{13} t^{7}+7 q^{14} t^{7}+8 q^{15} t^{7}+6 q^{16} t^{7}+q^{11} t^{8}+2 q^{12} t^{8}+4 q^{13} t^{8}+5 q^{14} t^{8}$ $+7 q^{15} t^{8}+8 q^{16} t^{8}+5 q^{17} t^{8}+q^{12} t^{9}+2 q^{13} t^{9}+4 q^{14} t^{9}+5 q^{15} t^{9}+7 q^{16} t^{9}+7 q^{17} t^{9}+3 q^{18} t^{9}$ $+q^{13} t^{10}+2 q^{14} t^{10}+4 q^{15} t^{10}+5 q^{16} t^{10}+7 q^{17} t^{10}+5 q^{18} t^{10}+q^{19} t^{10}+q^{14} t^{11}+2 q^{15} t^{11}$ $+4 q^{16} t^{11}+5 q^{17} t^{11}+6 q^{18} t^{11}+2 q^{19} t^{11}+q^{15} t^{12}+2 q^{16} t^{12}+4 q^{17} t^{12}+5 q^{18} t^{12}+4 q^{19} t^{12}$ $+q^{16} t^{13}+2 q^{17} t^{13}+4 q^{18} t^{13}+4 q^{19} t^{13}+q^{20} t^{13}+q^{17} t^{14}+2 q^{18} t^{14}+4 q^{19} t^{14}+2 q^{20} t^{14}$ $\left.+q^{18} t^{15}+2 q^{19} t^{15}+3 q^{20} t^{15}+q^{19} t^{16}+2 q^{20} t^{16}+q^{21} t^{16}+q^{20} t^{17}+q^{21} t^{17}+q^{21} t^{18}\right)$ $+a\left(q+q^{2}+q^{3}+q^{2} t+2 q^{3} t+3 q^{4} t+2 q^{5} t+q^{6} t+q^{3} t^{2}+2 q^{4} t^{2}+4 q^{5} t^{2}+4 q^{6} t^{2}+4 q^{7} t^{2}\right.$

$+2 q^{8} t^{2}+q^{9} t^{2}+q^{4} t^{3}+2 q^{5} t^{3}+4 q^{6} t^{3}+5 q^{7} t^{3}+6 q^{8} t^{3}+5 q^{9} t^{3}+4 q^{10} t^{3}+q^{11} t^{3}+q^{5} t^{4}$ $+2 q^{6} t^{4}+4 q^{7} t^{4}+5 q^{8} t^{4}+7 q^{9} t^{4}+7 q^{10} t^{4}+7 q^{11} t^{4}+2 q^{12} t^{4}+q^{6} t^{5}+2 q^{7} t^{5}+4 q^{8} t^{5}+5 q^{9} t^{5}$

$+7 q^{10} t^{5}+8 q^{11} t^{5}+9 q^{12} t^{5}+3 q^{13} t^{5}+q^{7} t^{6}+2 q^{8} t^{6}+4 q^{9} t^{6}+5 q^{10} t^{6}+7 q^{11} t^{6}+8 q^{12} t^{6}$ $+10 q^{13} t^{6}+3 q^{14} t^{6}+q^{8} t^{7}+2 q^{9} t^{7}+4 q^{10} t^{7}+5 q^{11} t^{7}+7 q^{12} t^{7}+8 q^{13} t^{7}+10 q^{14} t^{7}+3 q^{15} t^{7}$ $+q^{9} t^{8}+2 q^{10} t^{8}+4 q^{11} t^{8}+5 q^{12} t^{8}+7 q^{13} t^{8}+8 q^{14} t^{8}+10 q^{15} t^{8}+3 q^{16} t^{8}+q^{10} t^{9}+2 q^{11} t^{9}$

$+4 q^{12} t^{9}+5 q^{13} t^{9}+7 q^{14} t^{9}+8 q^{15} t^{9}+9 q^{16} t^{9}+2 q^{17} t^{9}+q^{11} t^{10}+2 q^{12} t^{10}+4 q^{13} t^{10}$ $+5 q^{14} t^{10}+7 q^{15} t^{10}+8 q^{16} t^{10}+7 q^{17} t^{10}+q^{18} t^{10}+q^{12} t^{11}+2 q^{13} t^{11}+4 q^{14} t^{11}+5 q^{15} t^{11}$ $+7 q^{16} t^{11}+7 q^{17} t^{11}+4 q^{18} t^{11}+q^{13} t^{12}+2 q^{14} t^{12}+4 q^{15} t^{12}+5 q^{16} t^{12}+7 q^{17} t^{12}+5 q^{18} t^{12}$ $+q^{19} t^{12}+q^{14} t^{13}+2 q^{15} t^{13}+4 q^{16} t^{13}+5 q^{17} t^{13}+6 q^{18} t^{13}+2 q^{19} t^{13}+q^{15} t^{14}+2 q^{16} t^{14}$ $+4 q^{17} t^{14}+5 q^{18} t^{14}+4 q^{19} t^{14}+q^{16} t^{15}+2 q^{17} t^{15}+4 q^{18} t^{15}+4 q^{19} t^{15}+q^{20} t^{15}+q^{17} t^{16}$ $+2 q^{18} t^{16}+4 q^{19} t^{16}+2 q^{20} t^{16}+q^{18} t^{17}+2 q^{19} t^{17}+3 q^{20} t^{17}+q^{19} t^{18}+2 q^{20} t^{18}+q^{21} t^{18}$ $\left.+q^{20} t^{19}+q^{21} t^{19}+q^{21} t^{20}\right)$. 
Versal deformations Last, but not least, we refer the reader to [24] for some relatively recent developments concerning the Hilbert schemes of (locally) plane curves, including their role in the theory of the Gopakumar-Vafa BPS invariants (see [35]) and various generalizations.

This paper is helpful to put our construction into perspective and to link it to the versal deformations of plane curve singularities, at least in the case of usual (nonnested) Hilbert schemes of curves. We do not touch this important direction here. However, let us mention that the theory of adjacent singularities of the pairs $\{R, V\}$ for finitedimensional irreducible representations $V$ of the simple Lie groups $G$ for (arbitrary) root systems $R$ is expected to be related to the theory of the DAHA superpolynomials.

When $t_{s t}=1$, and for the minimal possible degree of $a_{s t}$, the sum in (4-24) essentially reduces to the following summation:

$$
\sum_{n \geq 0} q_{s t}^{n+1-\delta} e\left(\mathcal{C}^{[n]}\right)=\sum_{0 \leq i \leq \delta} n_{\mathcal{C}}(i)\left(\frac{q_{s t}}{\left(1-q_{s t}\right)^{2}}\right)^{i+1-\delta}, \quad \mathcal{C}=\mathcal{C}_{\overrightarrow{\mathrm{r}}, \overrightarrow{\mathrm{s}}},
$$

for the Euler numbers of Hilbert schemes $\mathcal{C}^{[n]}$ of $n$ points on $\mathcal{C}$ of arithmetic genus $\delta$ (subschemes in $\mathcal{C}$ of length $n$, to be more exact). See [35; 24]. Importantly, $n_{\mathcal{C}}(i) \in \mathbb{Z}_{+}$ due to [18] and [42], because these numbers are the multiplicities of $\mathcal{C}$ in the closures of the strata in the space of its versal deformation, stratified with respect to the geometric genus of the deformation curves.

In our notations, $t_{s t}=1$ corresponds to $q=t$, and we can therefore use our $\sqrt{q}$ instead of $q_{s t}$ here upon $a=0$. Hopefully, [33, Conjecture 2] and our construction lead to a similar deformation interpretation of the generalization of the right-hand side of (4-25) to the nested Hilbert-schemes (which adds the parameter $a_{s t}$ ) under the weight filtration (associated with $t_{s t}$ ) and for arbitrary Young diagrams.

Acknowledgements Our special thanks go to Vivek Shende for clarifying discussions on the topics related to $[33 ; 24]$ and to Mikhail Khovanov for many conversations on his and Rozansky's theory. We thank Semen Artamonov for our use of his unique software for calculating colored HOMFLYPT polynomials, Peter Samuelson for sending us his work before it was posted, and him and Yuri Berest for discussions. The first author thanks Andras Szenes for the talks on the algebraic-geometric aspects of our construction and the University of Geneva for the invitation and hospitality. We thank the referees for their attention to our paper, thorough reports and important suggestions.

The paper was mainly written at RIMS. The first author thanks Hiraku Nakajima and RIMS for the invitation and hospitality and the Simons Foundation; the second author is 
grateful for the invitation to the school and conference "Geometric Representation Theory" at RIMS (July 21- August 1, 2014) and generous RIMS support. IC acknowledges partial support from the NSF grant DMS-1363138 and the Simons Foundation. ID acknowledges partial support from the RFBR grants 13-02-00478, 14-02-31446-mol-a, NSh-1500.2014.2 and the common grant 14-01-92691-Ind-a.

\section{References}

[1] M Aganagic, S Shakirov, Knot homology from refined Chern-Simons theory, preprint (2011) arXiv:1105.5117v1

[2] A Anokhina, A Mironov, A Morozov, A Morozov, Colored HOMFLY polynomials as multiple sums over paths or standard Young tableaux, Adv. High Energy Phys. (2013) Art. ID 931830 MR3127275

[3] A Anokhina, A Morozov, Cabling procedure for the colored HOMFLY polynomials, preprint (2014) arXiv:1307.2216

[4] A Beauville, Counting rational curves on K3 surfaces, Duke Math. J. 97 (1999) 99-108 MR1682284

[5] Y Berest, P Samuelson, Double affine Hecke algebras and generalized Jones polynomials, preprint (2014) arXiv:1402.6032

[6] F Bergeron, A Garsia, E Leven, G Xin, Some remarkable new plethystic operators in the theory of Macdonald polynomials, preprint (2014) arXiv:1405.0316

[7] N Bourbaki, Groupes et algèbres de Lie, 4-6, Actualités Scientifiques et Industrielles 1337, Hermann, Paris (1968) MR0240238

[8] I V Cherednik, Elliptic curves and matrix soliton differential equations, from: "Algebra, Topology, Geometry", (R V Gamkrelidze, editor), Itogi Nauki i Tekhniki 22, Akad. Nauk SSSR, Moscow (1984) 205-265 MR778566

[9] I Cherednik, Macdonald's evaluation conjectures and difference Fourier transform, Invent. Math. 122 (1995) 119-145 MR1354956

[10] I Cherednik, Double affine Hecke algebras, London Mathematical Society Lecture Note Series 319, Cambridge Univ. Press (2005) MR2133033

[11] I Cherednik, Nonsemisimple Macdonald polynomials, Selecta Math. 14 (2009) 427569 MR2511191

[12] I Cherednik, Jones polynomials of torus knots via DAHA, Int. Math. Res. Not. 2013 (2013) 5366-5425 MR3142259

[13] I Cherednik, DAHA-Jones polynomials of torus knots, preprint (2014) arXiv: 1406.3959 
[14] D-E Diaconescu, Z Hua, Y Soibelman, HOMFLY polynomials, stable pairs and motivic Donaldson-Thomas invariants, Commun. Number Theory Phys. 6 (2012) 517-600 MR3021320

[15] N M Dunfield, S Gukov, J Rasmussen, The superpolynomial for knot homologies, Experiment. Math. 15 (2006) 129-159 MR2253002

[16] P Dunin-Barkowski, A Mironov, A Morozov, A Sleptsov, A Smirnov, Superpolynomials for torus knots from evolution induced by cut-and-join operators, J. High Energy Phys. (2013) MR3046744

[17] D Eisenbud, W Neumann, Three-dimensional link theory and invariants of plane curve singularities, Ann. Math. Studies 110, Princeton Univ. Press (1985) MR817982

[18] B Fantechi, L Göttsche, D van Straten, Euler number of the compactified Jacobian and multiplicity of rational curves, J. Algebraic Geom. 8 (1999) 115-133 MR1658220

[19] H Fuji, S Gukov, P Sułkowski, Super-A-polynomial for knots and BPS states, Nuclear Phys. B 867 (2013) 506-546 MR2992793

[20] E Gorsky, S Gukov, M Stosic, Quadruply-graded colored homology of knots, preprint (2013) arXiv:1304.3481

[21] E Gorsky, M Mazin, Compactified Jacobians and q, $t$-Catalan numbers, I, J. Combin. Theory Ser. A 120 (2013) 49-63 MR2971696

[22] E Gorsky, A Negut, Refined knot invariants and Hilbert schemes, J. Math. Pures Appl. 104 (2015) 403-435 MR3383172

[23] E Gorsky, A Oblomkov, J Rasmussen, V Shende, Torus knots and the rational DAHA, Duke Math. J. 163 (2014) 2709-2794 MR3273582

[24] L Göttsche, V Shende, Refined curve counting on complex surfaces, Geom. Topol. 18 (2014) 2245-2307 MR3268777

[25] S Gukov, M Stošić, Homological algebra of knots and BPS states, from: "Proceedings of the Freedman Fest”, (R Kirby, V Krushkal, Z Wang, editors), Geom. Topol. Monogr. 18 (2012) 309-367 MR3084243

[26] M Khovanov, Triply-graded link homology and Hochschild homology of Soergel bimodules, Internat. J. Math. 18 (2007) 869-885 MR2339573

[27] M Khovanov, L Rozansky, Matrix factorizations and link homology, Fund. Math. 199 (2008) 1-91 MR2391017

[28] M Khovanov, L Rozansky, Matrix factorizations and link homology, II, Geom. Topol. 12 (2008) 1387-1425 MR2421131

[29] G Laumon, Fibres de Springer et jacobiennes compactifiées, from: "Algebraic geometry and number theory”, (V Ginzburg, editor), Progr. Math. 253, Birkhäuser, Boston (2006) 515-563 MR2263199 
[30] D Maulik, Stable pairs and the HOMFLY polynomial, preprint (2012) arXiv: 1210.6323

[31] J Milnor, Singular points of complex hypersurfaces, Ann. Math. Studies 61, Princeton Univ. Press; University of Tokyo Press (1968) MR0239612

[32] H R Morton, The coloured Jones function and Alexander polynomial for torus knots, Math. Proc. Cambridge Philos. Soc. 117 (1995) 129-135 MR1297899

[33] A Oblomkov, J Rasmussen, V Shende, The Hilbert scheme of a plane curve singularity and the HOMFLY homology of its link, preprint (2012) arXiv:1201.2115

[34] A Oblomkov, V Shende, The Hilbert scheme of a plane curve singularity and the HOMFLY polynomial of its link, Duke Math. J. 161 (2012) 1277-1303 MR2922375

[35] R Pandharipande, R P Thomas, Stable pairs and BPS invariants, J. Amer. Math. Soc. 23 (2010) 267-297 MR2552254

[36] J Piontkowski, Topology of the compactified Jacobians of singular curves, Math. Z. 255 (2007) 195-226 MR2262728

[37] J Rasmussen, Some differentials on Khovanov-Rozansky homology, Geom. Topol. 19 (2015) 3031-3104 MR3447099

[38] M Rosso, V Jones, On the invariants of torus knots derived from quantum groups, J. Knot Theory Ramifications 2 (1993) 97-112 MR1209320

[39] R Rouquier, Khovanov-Rozansky homology and 2-braid groups, preprint (2012) arXiv: 1203.5065

[40] P Samuelson, A topological construction of Cherednik's $\mathfrak{s l}_{2}$ torus knot polynomials, preprint (2015) arXiv: 1408.0483

[41] O Schiffmann, E Vasserot, The elliptic Hall algebra, Cherednik Hecke algebras and Macdonald polynomials, Compos. Math. 147 (2011) 188-234 MR2771130

[42] V Shende, Hilbert schemes of points on a locally planar curve and the Severi strata of its versal deformation, Compos. Math. 148 (2012) 531-547 MR2904196

[43] S Stevan, Chern-Simons invariants of torus links, Ann. Henri Poincaré 11 (2010) 1201-1224 MR2753387

[44] A T Tran, The strong AJ conjecture for cables of torus knots, J. Knot Theory Ramifications 24 (2015) 1550072 MR3434558

[45] M Varagnolo, E Vasserot, Finite-dimensional representations of DAHA and affine Springer fibers: the spherical case, Duke Math. J. 147 (2009) 439-540 MR2510742

[46] J M Wahl, Equisingular deformations of plane algebroid curves, Trans. Amer. Math. Soc. 193 (1974) 143-170 MR0419439

[47] B Webster, Knot invariants and higher representation theory, preprint (2015) arXiv: 1309.3796 
[48] B Webster, G Williamson, A geometric construction of colored HOMFLYPT homology, preprint (2010) arXiv:0905.0486

[49] Z Yun, Global Springer theory, Adv. Math. 228 (2011) 266-328 MR2822234

[50] O Zariski, Le problème des modules pour les branches planes, École Polytechnique, Paris (1973) MR0414561

IC: Department of Mathematics, University of North Carolina

Chapel Hill, NC 27599-3250, USA

ID: MIPT, 9 Institutskiy per.

Dolgoprudny, Moscow region, 141700, Russia

ID: ITEP, 25 Bolshaya Cheremushkinskaya

Moscow, 117218, Russia

chered@email.unc.edu, danilenko_i@mail.ru

Received: 19 December 2014 Revised: 5 June 2015 